\title{
Using $N$-nitrosodichloroacetamides to conveniently convert linear primary amines into alcohols
}

Nicholas S. MacArthur, Linshu Wang, Blaine G. McCarthy, Charles E. Jakobsche*

Carlson School of Chemistry \& Biochemistry, Clark University, Worcester, MA

Email: cjakobsche@clarku.edu

Table of Contents

1. Additional Data from the Rearrangement and Nitrosylation Reactions S2

(Supporting Tables S1-S3)

2. Synthetic Procedures S4

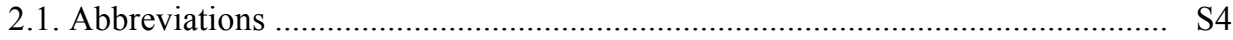

2.2. General Procedures ................................................................................... S4

2.3. Standard Amine-to-Alcohol Conversion Procedure ………………………......... S5

2.4 Conversion of Amine 1 to Alcohol 5 ............................................................. S6

(as an Example of the Standard Procedure)

2.5 Amine-to-Alcohol Reactions from the Substrate Scope Table .......................... S8

2.6 Synthesis of Amine Substrates from the Substrate Scope Table ........................ S11

2.7 Formation of Imidoyl Trifluoroacetate $\mathbf{2 8}$.................................................... S15

2.8 Synthesis of Amide Substrates for Mechanistic Studies and Table S3 .............. S16

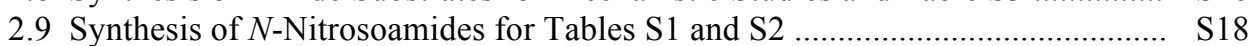

2.10 Thermal Rearrangements of $N$-Nitrosoamides for Tables S1 and S2 ............... S20

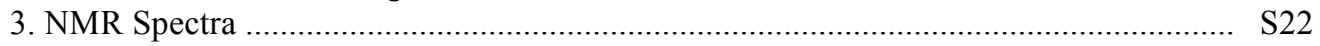




\section{Additional Data from the Rearrangement and Nitrosylation Reactions}

Table S1: Thermal rearrangements of some $N$-nitrosoamides.

standard
standard

Conditions: $0.1 \mathrm{M}$ in toluene or the indicated solvent, warmed for the indicated time and temperature, solvent removed, conversion and product distribution measured by ${ }^{1} \mathrm{H}$ NMR of the crude product.

Table S2: $N$-nitrosoamide rearrangements that produce additional byproducts.

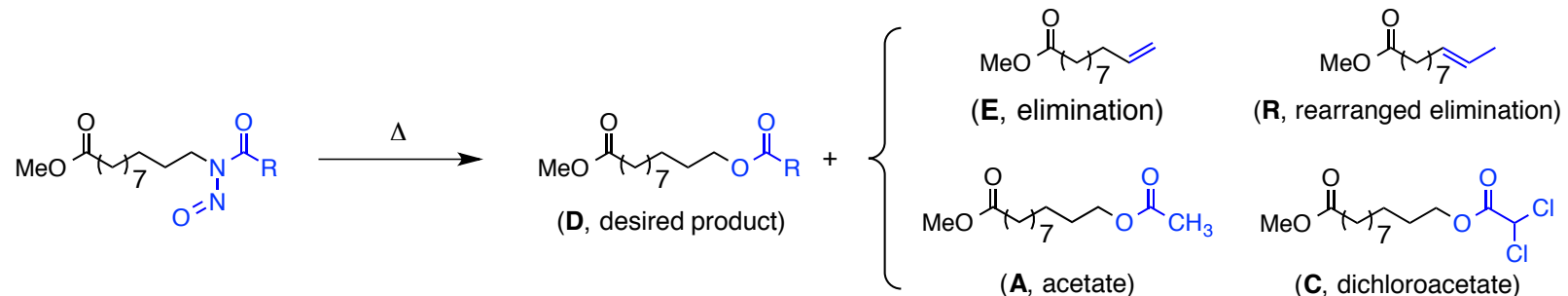

\begin{tabular}{|c|c|c|c|c|c|c|}
\hline Nitrosoamide & Entry & Conditions & Temperature & Time & Conversion & Product Distribution \\
\hline & 1 & toluene (standard) & $50^{\circ} \mathrm{C}$ & $24 \mathrm{~h}$ & full & $88(D): 12(E)$ \\
\hline & 2 & toluene +1 equiv TEA & $50^{\circ} \mathrm{C}$ & $24 \mathrm{~h}$ & full & 89 (D) : 11 (E) \\
\hline & 3 & toluene +1 equiv $\mathrm{AcOH}$ & $50^{\circ} \mathrm{C}$ & $24 \mathrm{~h}$ & full & $53(\mathbf{D}): 36(\mathbf{A}): 11(\mathrm{E})$ \\
\hline S3 & 4 & toluene +1 equiv $\mathrm{AcOH}$ and TEA & $50^{\circ} \mathrm{C}$ & $24 \mathrm{~h}$ & full & $53($ D) $: 36(\mathbf{A}): 11(\mathbf{E})$ \\
\hline & 5 & $5: 1 \mathrm{Ac}_{2} \mathrm{O} / \mathrm{AcOH}$ & \multicolumn{2}{|c|}{$0^{\circ} \mathrm{C}$ during nitrosylation } & full & $53(\mathbf{A}): 22(\mathbf{E}): 10(\mathbf{R}): 15(\mathbf{D})$ \\
\hline & 6 & $5: 1 \mathrm{Ac}_{2} \mathrm{O} / \mathrm{DCA}$ & \multicolumn{2}{|c|}{$0^{\circ} \mathrm{C}$ during nitrosylation } & full & $36(\mathbf{C}): 23(\mathbf{A}): 22(\mathbf{E}): 7(\mathbf{R}): 12(\mathbf{D})$ \\
\hline & 7 & $5: 1 \mathrm{Ac}_{2} \mathrm{O} / \mathrm{TFA}$ & \multicolumn{2}{|c|}{$0^{\circ} \mathrm{C}$ during nitrosylation } & full & $64(\mathbf{D}): 15(\mathbf{A}): 17(\mathbf{E}): 4(\mathbf{R})$ \\
\hline S4 & 8 & 5:1 TFAA / TFA & \multicolumn{2}{|c|}{$0^{\circ} \mathrm{C}$ during nitrosylation } & full & messy: approx. 50 (D) : 50 (other) \\
\hline
\end{tabular}

Conditions: For entries 1-4: $0.1 \mathrm{M}$ in the indicated solvent, warmed for the indicated time and temperature, solvent removed, crude product analyzed. For entries 5-8: crude product from the nitrosylation reaction analyzed. Conversion and product distribution measured by ${ }^{1} \mathrm{H}$ NMR. Abbreviations: $\mathrm{DCA}=$ dichloroacetic acid, $\mathrm{TFA}=$ trifluoroacetic acid, TFAA $=$ trifluoroacetic anhydride. 
Table S3: $N$-Nitrosylation of some amides.

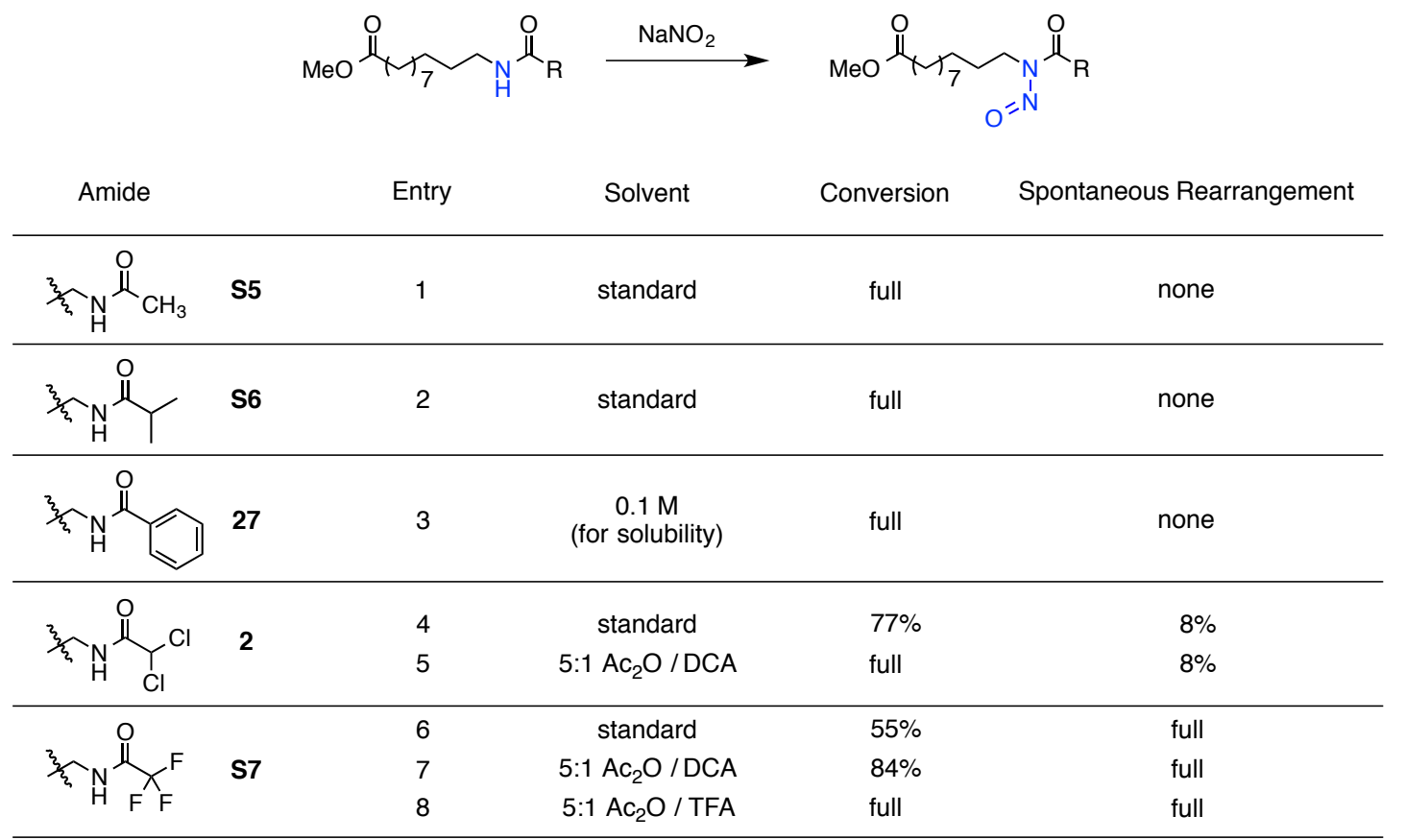

Conditions: $0.3 \mathrm{M}$ in 5:1 acetic anhydride / acetic acid or indicated solvent mixture, dry, $0{ }^{\circ} \mathrm{C}, 2$ equiv $\mathrm{NaNO}_{2}, 2 \mathrm{~h}$, additional 2 equiv $\mathrm{NaNO}_{2}$, additional $4 \mathrm{~h}$, conversion and product distribution measured by ${ }^{1} \mathrm{H}$ NMR of the crude product. Abbreviations: $\mathrm{DCA}=$ dichloroacetic acid, TFA $=$ trifluoroacetic acid. 


\section{Synthetic Procedures}

\subsection{Abbreviations}

$\mathrm{DCM}=$ dichloromethane

$\mathrm{DMF}=N, N$-dimethylformamide

DMSO = dimethylsulfoxide

EDC hydrochloride $=\mathrm{N}$-(3-dimethylaminopropyl)-

N'-ethylcarbodiimide hydrochloride

Ether $=$ diethyl ether

EtOAc $=$ ethyl acetate
HOBt hydrate $=$ hydroxybenzotriazole hydrate

$\mathrm{MeOH}=$ methanol

Quant $=$ quantitative conversion

TEA $=$ triethylamine

TFA $=$ trifluoroacetic acid

$\mathrm{THF}=$ tetrahydrofurane

$\mathrm{y}=$ yield

\subsection{General Procedures}

Column chromatography was performed with $60 \AA 40-63 \mu \mathrm{m}$ silia-P flash silica gel.

Solvents for reactions (DMF, DCM, THF, and toluene) were dried using a LC Technology Solutions purification system. Other solvents were used as received unless noted otherwise.

Chemicals were purchased from Fisher, VWR, or Sigma-Aldrich and used as received unless noted otherwise.

NMR Spectra were measured in $\mathrm{CDCl}_{3}$ at ambient temperature unless otherwise noted.

${ }^{1} \mathrm{H}$ NMR spectra were recorded on either a 600 or $200 \mathrm{MHz}$ Varian spectrometer. Chemical shifts are reported in ppm $(\delta)$ relative to tetramethylsilane using the solvent as a reference $\left(\mathrm{CDCl}_{3}=7.26 \mathrm{ppm}\right.$, DMSO- $d_{6}=2.49 \mathrm{ppm}$, $\mathrm{D}_{2} \mathrm{O}=4.80 \mathrm{ppm}, \mathrm{CD}_{3} \mathrm{OD}=3.30$ ). The following is an example data point: chemical shift (multiplicity [s = singlet, $\mathrm{d}$ $=$ doublet, $\mathrm{t}=$ triplet, $\mathrm{q}=$ quartet, pent $=$ pentet, sext $=$ sextet, $\mathrm{sept}=$ septet, oct $=$ octet, $\mathrm{m}=$ multiplet, $\mathrm{br}=$ broad, and combinations thereof], coupling constants [Hz], integration, assignment [if any]).

${ }^{13} \mathrm{C}$ NMR spectra were recorded on a 600 or $200 \mathrm{MHz}(150$ or $50 \mathrm{MHz})$ Varian spectrometer with complete proton decoupling. Chemical shifts are reported in $\mathrm{ppm}(\delta)$ relative to tetramethylsilane using the solvent or $\mathrm{MeOH}$ as a reference $\left(\mathrm{CDCl}_{3}=77.0 \mathrm{ppm}, \mathrm{DMSO}-d_{6}=39.5 \mathrm{ppm}, \mathrm{CD}_{3} \mathrm{OD}=49.0 \mathrm{ppm}, \mathrm{MeOH}=49.5\right)$.

IR spectra were recorded on a Perkin Elmer Spectrum 100 FT-IR spectrometer with Perkin Elmer Spectrum software. Spectra are partially reported $\left(v_{\max }, \mathrm{cm}^{-1}\right)$.

HRMS were obtained at The University of Illinois Urbana-Champagne.

TLC was performed on $60 \AA \mathrm{F}_{254}$ pre-coated silica gel plates. Samples were visualized by either ultraviolet irradiation, potassium permanganate staining, or cerium ammonium molybdenate staining.

Yield refers to isolated material.

Quantitative recovery means that mostly pure material was recovered in approximately the expected mass, and the material was used directly for the next step without purification. 


\subsection{Standard Amine-to-Alcohol Conversion Procedure}

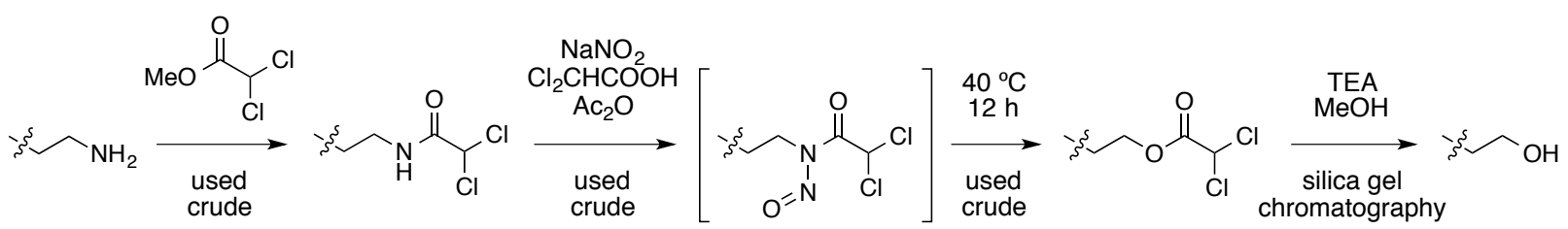

Standard dichloroacetylation procedure Into a flask were added the starting free amine (1 equiv), distilled EtOAc $(1 \mathrm{M})$, and methyl dichloroacetate (1.5 equiv). The mixture was stirred at ambient temperature overnight. Volatiles were removed under reduced pressure to yield the dichloroacetamide, which was used directly for the subsequent reaction.

Standard nitrosylation procedure The crude dichloroacetamide was dissolved in the required solvent mixture (typically $0.3 \mathrm{M}$ in 1:1 acetic anhydride / dichloroacetic acid). The flask was capped with a rubber septum and stirred in an ice bath $\left(0{ }^{\circ} \mathrm{C}, 30 \mathrm{~min}\right)$. To the mixture was added solid sodium nitrite ( 2 equiv, creating a blue solution and slowly evolving brown gas). The flask was capped with a rubber septum and vented with a syringe packed with anhydrous calcium sulfate, and stirred $(2 \mathrm{~h})$. To the mixture was added additional sodium nitrite ( 2 equiv). The mixture was stirred (additional $4 \mathrm{~h}$ ), diluted with EtOAc $(0.1 \mathrm{M})$, washed with cold water (equal volume) and then cold sodium carbonate ( $10 \%$ aqueous, $3 \times$ equal volume), diluted with cold sodium carbonate (10\% aqueous, equal volume), and stirred rapidly (30 min, to quench any remaining anhydride). The organic phase was isolated, washed with potassium phosphate monobasic ( $1 \mathrm{M}$ aqueous, $\mathrm{pH} 5$, equal volume), dried with sodium sulfate, and used directly for the subsequent transformation without removing the solvent.

Standard rearrangement procedure The solution of the crude $N$-nitrosoamide was warmed in an oil bath (40 ${ }^{\circ} \mathrm{C}, 24 \mathrm{~h}$ ). Volatiles were removed under reduced pressure to yield the crude dichloroacetate ester, which was used directly for the subsequent transformation.

Standard hydrolysis procedure The crude dichloroacetate ester was dissolved in $\mathrm{MeOH}(0.2 \mathrm{M})$, and TEA (2 equiv) was added. The mixture was stirred $(2 \mathrm{~h})$, diluted with potassium phosphate monobasic (1 M aqueous, $\mathrm{pH} 5$, $0.5 \mathrm{M})$, stirred $(10 \mathrm{~min})$, further diluted with water, extracted with EtOAc, and dried with sodium sulfate. Volatiles were removed under reduced pressure to yield a crude alcohol, which was purified by silica gel chromatography. 


\subsection{Conversion of Amine 1 to Alcohol 5 (as an Example of the Standard Procedure)}

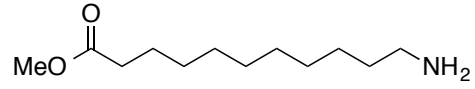

1

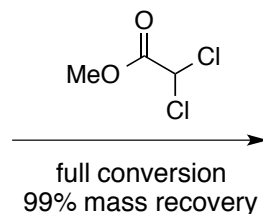

2a

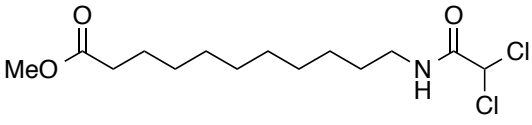

2

Synthesis of dichloroacetamide 2 Into a flask were added ester 1 (1.10 g, 5.10 mmol, 1 equiv), EtOAc (distilled, $5 \mathrm{~mL}, 1 \mathrm{M}$ ), and methyl dichloroacetate $(\mathbf{2 a}, 0.79 \mathrm{~mL}, 7.6 \mathrm{mmol}, 1.5$ equiv). The mixture was stirred at ambient temperature $(21 \mathrm{~h})$. Volatiles were removed under reduced pressure to yield crude amide 2 (1.64 g, full conversion, 99\% mass recovery, white solid), which was used directly for the subsequent reaction. For analysis, an aliquot was purified by column chromatography (silica gel, 5:1 hexane/EtOAc) to yield pure amide 2.

${ }^{1} \mathbf{H}$ NMR $\left(\mathrm{CDCl}_{3}, 200 \mathrm{MHz}\right) \delta 6.50(\mathrm{brs}, 1 \mathrm{H}, \mathrm{NH}), 5.92\left(\mathrm{~s}, 1 \mathrm{H}, \mathrm{Cl}_{2} \mathrm{CH}\right), 3.67(\mathrm{~s}, 3 \mathrm{H}, \mathrm{OMe}), 3.33(\mathrm{q}, J=6.9 \mathrm{~Hz}$, $\left.2 \mathrm{H}, \mathrm{N}-\mathrm{CH}_{2}\right), 2.30\left(\mathrm{t}, J=7.5 \mathrm{~Hz}, 2 \mathrm{H}, \mathrm{CH}_{2} \mathrm{C}=\mathrm{O}\right), 1.70-1.47\left(\mathrm{~m}, 4 \mathrm{H}, 2 \times \mathrm{CH}_{2}\right), 1.40-1.16\left(\mathrm{~m}, 12 \mathrm{H},\left(\mathrm{CH}_{2}\right)_{6}\right)$.

${ }^{13} \mathrm{C} \mathrm{NMR}\left(\mathrm{CDCl}_{3}, 50 \mathrm{MHz}\right) \delta 174.31,163.98,66.48,51.44,40.35,34.06,29.31,29.23,29.14,29.08,29.05$, $26.62,24.88$.

IR (neat, $\mathrm{cm}^{-1}$ ) 3267, 3090, 2919, 2851, 1720, 1662, 1559, 1166, 812, 655.

TLC (3:1) hexane/EtOAc, permanganate, $R_{f}=0.52$.

HRMS calculated for $\left[\mathrm{C}_{14} \mathrm{H}_{25} \mathrm{Cl}_{2} \mathrm{NO}_{3} \mathrm{H}\right]^{+}$, requires $\mathrm{m} / z=326.1290$, found $m / z=326.1290$ (ESI).<smiles>COC(=O)CCCCNC(=O)C(Cl)Cl</smiles>

2

3

4

Synthesis of $\boldsymbol{N}$-nitrosoamide 3 Into a flask were added crude amide 2 (5.04 mmol, 1 equiv) and a 1:1 mixture of dichloroacetic acid and acetic anhydride, $(16 \mathrm{~mL}, 0.3 \mathrm{M})$. The flask was capped with a rubber septum, sealed with tape, and cooled in an ice bath $\left(0^{\circ} \mathrm{C}, 30 \mathrm{~min}\right)$. To the mixture was added sodium nitrite $(696 \mathrm{mg}, 10.1 \mathrm{mmol}, 2$ equiv, producing a blue solution and slowly evolving some brown gas). The flask was capped with a rubber septum, sealed with tape, vented through a syringe packed with anhydrous calcium sulfate, and stirred in an ice bath $\left(0{ }^{\circ} \mathrm{C}, 2\right.$ h). To the mixture was added additional sodium nitrite $(696 \mathrm{mg}, 10.1 \mathrm{mmol}, 2$ equiv). The mixture was stirred in an ice bath $\left(0{ }^{\circ} \mathrm{C}\right.$, additional $\left.4 \mathrm{~h}\right)$, diluted with EtOAc $(40 \mathrm{~mL})$, and washed with cold water $(40 \mathrm{~mL})$ and then cold sodium carbonate $(10 \%$ aqueous, $50+2 \times 40 \mathrm{~mL})$. To quench any remaining anhydride, the organic phase was mixed with sodium carbonate $(10 \%$ aqueous, $40 \mathrm{~mL})$, maintained in an ice bath $\left(0^{\circ} \mathrm{C}\right)$, and stirred rapidly $(30 \mathrm{~min})$. The organic phase was isolated, washed with potassium phosphate monobasic (1 M aqueous, pH 5, $3 \mathrm{x} 40 \mathrm{~mL}$ ), and dried with sodium sulfate. The crude solution of $N$-nitrosoamide $\mathbf{3}$ was used directly for the subsequent transformation. An aliquot was removed for analysis. Volatiles were removed under reduced pressure from this aliquot, but since the product is not fully stable to silica gel or prolonged storage, analytical data was collected from the crude sample.

${ }^{1} \mathbf{H}$ NMR $\left(\mathrm{CDCl}_{3}, 200 \mathrm{MHz}\right.$, major signals) $\delta 7.23\left(\mathrm{~s}, 1 \mathrm{H}, \mathrm{CHCl}_{2}\right), 3.79\left(\mathrm{t}, J=7.3,2 \mathrm{H}, \mathrm{N}-\mathrm{CH}_{2}\right), 3.67(\mathrm{~s}, 3 \mathrm{H}$, $\mathrm{OMe}), 2.30\left(\mathrm{t}, J=7.5 \mathrm{~Hz}, 2 \mathrm{H}, \mathrm{O}=\mathrm{C}-\mathrm{CH}_{2}\right), 1.72-1.48\left(\mathrm{~m}, 2 \mathrm{H}, \mathrm{CH}_{2}\right), 1.48-1.10\left(\mathrm{~m}, 14 \mathrm{H},\left(\mathrm{CH}_{2}\right)_{7}\right)$.

${ }^{13} \mathrm{C}$ NMR $\left(\mathrm{CDCl}_{3}, 50 \mathrm{MHz}\right.$, major signals) $\delta 174.22,167.01,63.79,51.34,39.76,33.94,29.07,28.81,26.48$, $26.29,24.78$

IR (neat, $\left.\mathrm{cm}^{-1}\right)$ 2928, 2854, 1735, 1528, 806.

TLC (3:1, major spot) hexane/Et ${ }_{2} \mathrm{O}, \mathrm{UV} \&$ permanganate, $\mathrm{R}_{f}=0.71$.

HRMS decomposes to ester 4.

Synthesis of Ester 4 The solution of crude $N$-nitrosoamide 3 ( $0.1 \mathrm{M}$ in EtOAc) was warmed in an oil bath (40 ${ }^{\circ} \mathrm{C}, 12 \mathrm{~h}$ ). Volatiles were removed under reduced pressure to yield crude ester 4 ( $1.61 \mathrm{~g}, 98 \%$ mass recovery, full consumption of starting material, 7:1 rearrangement / elimination, yellow oil), which was used directly in the 
subsequent reaction. For analysis, an aliquot was purified by column chromatography (silica gel, 10:1 hexane/Et ${ }_{2} \mathrm{O}$ ) to yield pure ester 4 . The product ratio was determined by integration of the ${ }^{1} \mathrm{H}$ NMR spectrum of the crude material.

${ }^{1} \mathbf{H}$ NMR $\left(\mathrm{CDCl}_{3}, 200 \mathrm{MHz}\right) \delta 5.94\left(\mathrm{~s}, 1 \mathrm{H}, \mathrm{Cl}_{2} \mathrm{CH}\right), 4.27\left(\mathrm{t}, J=6.6 \mathrm{~Hz}, 2 \mathrm{H}, \mathrm{O}-\mathrm{CH}_{2}\right), 3.67(\mathrm{~s}, 3 \mathrm{H}, \mathrm{OMe}), 2.30$ $\left(\mathrm{t}, J=7.5 \mathrm{~Hz}, 2 \mathrm{H}, \mathrm{O}=\mathrm{C}-\mathrm{CH}_{2}\right), 1.79-1.53\left(\mathrm{~m}, 4 \mathrm{H}, 2 \times \mathrm{CH}_{2}\right), 1.46-1.17\left(\mathrm{~m}, 12 \mathrm{H},\left(\mathrm{CH}_{2}\right)_{6}\right)$.

${ }^{13} \mathrm{C}$ NMR $\left(\mathrm{CDCl}_{3}, 50 \mathrm{MHz}\right) \delta 174.29,164.59,67.70,64.33,51.44,34.06,29.31,29.25,29.16,29.07,29.03$, $28.21,25.55,24.89$.

IR (neat, $\mathrm{cm}^{-1}$ ) 2927, 2855, 1763, 1737, 1463, 1436, 1279, 1166, 814.

TLC (8:1) hexane/ $\mathrm{Et}_{2} \mathrm{O}$, permanganate, $\mathrm{R}_{f}=0.41$.

HRMS calculated for $\left[\mathrm{C}_{14} \mathrm{H}_{24} \mathrm{Cl}_{2} \mathrm{O}_{4} \mathrm{H}\right]^{+}$, requires $m / z=327.1130$, found $m / z=327.1129$ (ESI).
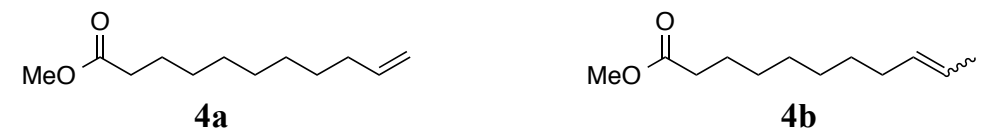

Terminal alkene $\mathbf{4 a}$ is the major elimination product, and a trace amount of internal alkene $\mathbf{4 b}$ is also observed. For analysis, an aliquot was purified by column chromatography. An inseparable mixture of the alkenes was isolated (silica gel, 10:1 hexane/Et ${ }_{2} \mathrm{O}$, co-eluting). Compound analysis is consistent with published data. ${ }^{1}$

Terminal alkene 4a:

${ }^{1}$ H NMR (major signals) $\left(\mathrm{CDCl}_{3}, 200 \mathrm{MHz}\right) \delta 5.81$ (ddt, $\left.1 \mathrm{H}, J_{t}=6.6 \mathrm{~Hz}, J_{d}=10.1,16.7 \mathrm{~Hz}, \mathrm{CH}=\mathrm{C}\right), 5.06-4.87$ $\left(\mathrm{m}, 2 \mathrm{H}, \mathrm{CH}_{2}=\mathrm{C}\right), 3.67(\mathrm{~s}, 3 \mathrm{H}, \mathrm{OMe}), 2.31\left(\mathrm{t}, J=7.5 \mathrm{~Hz}, 2 \mathrm{H}, \mathrm{O}=\mathrm{C}-\mathrm{CH}_{2}\right), 2.04\left(\mathrm{q}, J=6.5 \mathrm{~Hz}, 2 \mathrm{H}, \mathrm{CH}_{2}-\mathrm{C}=\mathrm{C}\right), 1.76-$ $1.50\left(\mathrm{~m}, 2 \mathrm{H}, \mathrm{CH}_{2}\right), 1.50-1.07\left(\mathrm{~m}, 10 \mathrm{H},\left(\mathrm{CH}_{2}\right)_{5}\right)$.

TLC (10:1) hexane/Et ${ }_{2} \mathrm{O}$, permanganate, $\mathrm{R}_{f}=0.49$.

Internal alkene $\mathbf{4 b}$ :

${ }^{1} \mathbf{H}$ NMR (minor signals) $\left(\mathrm{CDCl}_{3}, 200 \mathrm{MHz}\right) \delta 5.46-5.35(\mathrm{~m}, 2 \mathrm{H}, \mathrm{CH}=\mathrm{CH})$, other signals overlap with those of terminal alkene $\mathbf{4 a}$.

TLC $(10: 1)$ hexane/Et ${ }_{2} \mathrm{O}$, permanganate, $\mathrm{R}_{f}=0.49$ (overlapping alkene 4a).<smiles>COC(=O)CCCCCCCCCOC(=O)C(C)Cl</smiles>

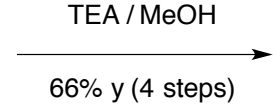

$66 \%$ y (4 steps)

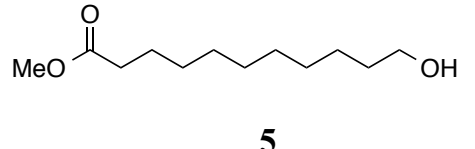

5

Synthesis of alcohol 5 Into a flask were added crude ester 4 (4.92 mmol, 1 equiv), $\mathrm{MeOH}$ ( $25 \mathrm{~mL}, 0.2 \mathrm{M}$ ), and TEA ( $1.40 \mathrm{~mL}, 9.84 \mathrm{mmol}, 2$ equiv). The mixture was stirred $(2 \mathrm{~h})$, diluted with potassium phosphate monobasic (1 $\mathrm{M}$ aqueous, $\mathrm{pH} 5,10 \mathrm{~mL})$ and water $(25 \mathrm{~mL})$, extracted with EtOAc $(40+20+10 \mathrm{~mL})$, and dried with sodium sulfate. Volatiles were removed under reduced pressure, and the crude material was purified by column chromatography (50 mL silica gel, [3:1 to 2:1] hexane/EtOAc) to yield alcohol $\mathbf{5}(0.721 \mathrm{~g}, 3.35 \mathrm{mmol}, 66 \%$ yield from amine 1, colorless oil). Compound analysis is consistent with published data. ${ }^{2}$

${ }^{1} \mathbf{H}$ NMR $\left(\mathrm{CDCl}_{3}, 200 \mathrm{MHz}\right) \delta 3.67(\mathrm{~s}, 3 \mathrm{H}, \mathrm{OMe}), 3.67-3.59\left(\mathrm{~m}, 2 \mathrm{H}, \mathrm{O}-\mathrm{CH}_{2}\right), 2.30\left(\mathrm{t}, J=7.5, \mathrm{O}=\mathrm{C}-\mathrm{CH}_{2}\right)$, $1.70-1.47\left(\mathrm{~m}, 4 \mathrm{H}, 2 \times \mathrm{CH}_{2}\right), 1.42-1.20\left(\mathrm{~m}, 12 \mathrm{H},\left(\mathrm{CH}_{2}\right)_{6}\right)$.

TLC (2:1) hexane/EtOAc, permanganate, $\mathrm{R}_{f}=0.36$.

\footnotetext{
${ }^{1}$ Compound data from: Condorelli, G. G.; Motta, A.; Fragala, I. L.; Giannazzo, F.; Raineri, V.; Caneschi, A.; Gatteschi, D. Angew. Chem., Int. Ed. 2004, 43, 4081-4084.

${ }^{2}$ Compound data from: Miyamoto, K.; Sei, Y.; Yamaguchi, K.; Ochiai, M. J. Am. Chem. Soc. 2009, 131, 13821383 .
} 


\subsection{Amine-to-Alcohol Reactions from the Substrate Scope Table}

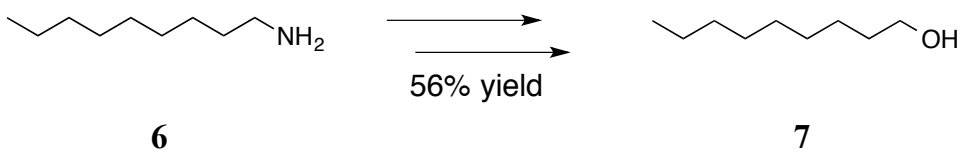

Synthesis of 1-nonanol (7) The standard procedure was followed beginning with nonylamine (6, $500 \mathrm{mg}, 3.49$ mmol, 1 equiv). To minimize loss of material into the aqueous washes, the workup of the nitrosylation step was modified to use 1:1 mixtures of brine and 10\% sodium carbonate for the base washes and a 1:1 mixture of brine and water for the final wash. The crude product was purified by column chromatography $\left(3: 2\right.$ hexane/Et ${ }_{2} \mathrm{O}, 40 \mathrm{~mL}$ silica gel) to yield 1-nonanol (7, $282 \mathrm{mg}, 1.97 \mathrm{mmol}, 56 \%$ yield). Prolonged exposure to high vacuum was avoided due to the product's moderate volatility $\left(\mathrm{BP}=214^{\circ} \mathrm{C}\right)$. Compound analysis is consistent with data from commercially available material. $^{3}$

${ }^{1} \mathrm{H}$ NMR $\left(\mathrm{CDCl}_{3}, 200 \mathrm{MHz}\right) \delta 3.64\left(\mathrm{t}, J=6.5 \mathrm{~Hz}, 2 \mathrm{H}, \mathrm{O}-\mathrm{CH}_{2}\right), 1.66-1.46\left(\mathrm{~m}, 2 \mathrm{H}, \mathrm{O}-\mathrm{C}-\mathrm{CH}_{2}\right), 1.43-1.12(\mathrm{~m}$, $\left.12 \mathrm{H},\left(\mathrm{CH}_{2}\right)_{6}\right), 0.89\left(\mathrm{t}, J=6.9 \mathrm{~Hz}, 3 \mathrm{H}, \mathrm{CH}_{3}\right)$.

TLC (3:2) hexane/ $\mathrm{Et}_{2} \mathrm{O}$, permanganate, $\mathrm{R}_{f}=0.34$.

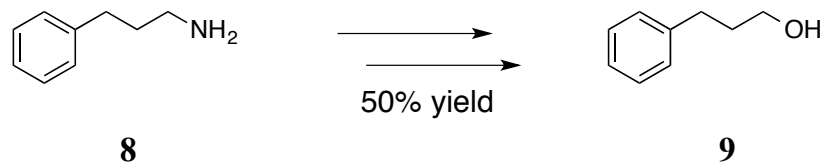

Synthesis of alcohol 9 The standard procedure was followed beginning with 3-phenylpropylamine $(\mathbf{8}, 500 \mathrm{mg}$, $3.70 \mathrm{mmol}, 1$ equiv). The crude product was purified by column chromatography $\left(1: 1\right.$ hexane/Et ${ }_{2} \mathrm{O}, 40 \mathrm{~mL}$ silica gel) to yield alcohol $9(254 \mathrm{mg}, 1.88 \mathrm{mmol}, 50 \%$ yield). Compound analysis is consistent with data from commercially available material. ${ }^{4}$

${ }^{1} \mathbf{H}$ NMR $\left(\mathrm{CDCl}_{3}, 200 \mathrm{MHz}\right) \delta 7.34-7.14(\mathrm{~m}, 5 \mathrm{H}, \mathrm{Ar}), 3.68\left(\mathrm{t}, J=6.3,2 \mathrm{H}, \mathrm{O}-\mathrm{CH}_{2}\right), 2.72(\mathrm{t}, J=7.6 \mathrm{~Hz}, 2 \mathrm{H}$, Ar- $\mathrm{CH}_{2}$ ), 1.91 (pent, $\left.J=7.0,2 \mathrm{H}, \mathrm{CH}_{2}\right), 1.26$ (brs, $1 \mathrm{H}, \mathrm{OH}$ ).

TLC (1:1) hexane $/ \mathrm{Et}_{2} \mathrm{O}$, permanganate, $\mathrm{R}_{f}=0.31$.

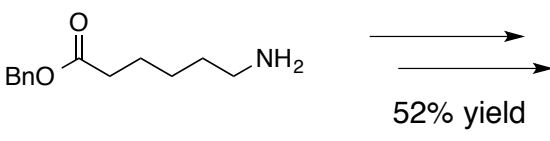

10

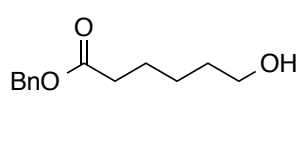

11

Synthesis of alcohol 11 The standard procedure was followed beginning with amine $\mathbf{1 0}$ (491 $\mathrm{mg}, 2.22 \mathrm{mmol}, 1$ equiv). The crude product was purified by column chromatography (2:1 hexane/EtOAc, $40 \mathrm{~mL}$ silica gel) to yield alcohol 11 (256 mg, $1.16 \mathrm{mmol}, 52 \%$ yield). Compound analysis is consistent with published data. ${ }^{5}$

${ }^{1} \mathbf{H}$ NMR $\left(\mathrm{CDCl}_{3}, 200 \mathrm{MHz}\right) \delta 7.39-7.31(\mathrm{~m}, 5 \mathrm{H}, \mathrm{Ar}), 5.12(\mathrm{~s}, 2 \mathrm{H}, \mathrm{Bn}), 3.64\left(\mathrm{t}, J=6.4 \mathrm{~Hz}, 2 \mathrm{H}, \mathrm{CH}_{2}-\mathrm{O}\right), 2.38$ $\left(\mathrm{t}, J=7.4 \mathrm{~Hz}, 2 \mathrm{H}, \mathrm{O}=\mathrm{C}-\mathrm{CH}_{2}\right), 1.79-1.24\left(\mathrm{~m}, 6 \mathrm{H},\left(\mathrm{CH}_{2}\right)_{3}\right)$.

TLC (2:1) hexane/EtOAc, permanganate, $\mathrm{R}_{f}=0.18$.

\footnotetext{
${ }^{3}$ Compound data from: www.sigmaaldrich.com

${ }^{4}$ Compound data from: Chen, G.; Fu, C.; Ma, S. Tetrahedron 2006, 62, 4444-4452.

${ }^{5}$ Compound data from: Samori, C.; Ali-Boucetta, H.; Sainz, R.; Guo, C.; Toma, F.; Fabbro, C.; de Ros, T.; Prato, M.; Kostarelos, K.; Bianco, A. Chem. Comm. 2010, 46, 1494-1496.
} 


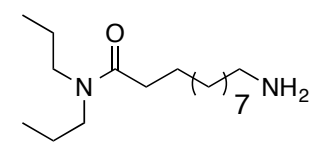

12

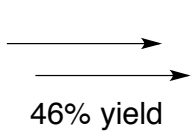

$46 \%$ yield

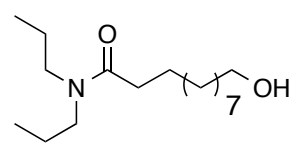

13

Synthesis of alcohol 13 The standard procedure was followed beginning with amine $12(1.06 \mathrm{~g}, 3.71 \mathrm{mmol}, 1$ equiv). The crude product was purified by column chromatography (2:1 EtOAc/hexane, $40 \mathrm{~mL}$ silica gel) to yield alcohol 13 (487 mg, $1.71 \mathrm{mmol}, 46 \%$ yield).

${ }^{1} \mathbf{H}$ NMR $\left(\mathrm{CDCl}_{3}, 200 \mathrm{MHz}\right) \delta 3.64\left(\mathrm{t}, J=6.6 \mathrm{~Hz}, 2 \mathrm{H}, \mathrm{O}-\mathrm{CH}_{2}\right), 3.32-3.13\left(\mathrm{~m}, 4 \mathrm{H}, \mathrm{CH}_{2}-\mathrm{N}-\mathrm{CH}_{2}\right), 2.28(\mathrm{t}, J=$ $\left.7.5 \mathrm{~Hz}, 2 \mathrm{H}, \mathrm{O}=\mathrm{C}-\mathrm{CH}_{2}\right), 1.76-1.44\left(\mathrm{~m}, 6 \mathrm{H}, 2 \times \mathrm{CH}_{2}-\mathrm{C}-\mathrm{N}, \mathrm{CH}_{2}-\mathrm{C}-\mathrm{O}\right), 1.44-1.20\left(\mathrm{~m}, 14 \mathrm{H},\left(\mathrm{CH}_{2}\right)_{7}\right), 0.90(\mathrm{q}, J=7.5$ $\left.\mathrm{Hz}, 6 \mathrm{H}, 2 \times \mathrm{CH}_{3}\right)$.

${ }^{13} \mathbf{C}$ NMR $\left(\mathrm{CDCl}_{3}, 50 \mathrm{MHz}\right) \delta 172.58,62.10,49.30,47.12,32.75,32.39,29.07,25.46,25.16,21.92,20.55$, $10.94,10.84$.

IR (neat, $\left.\mathrm{cm}^{-1}\right) 3428,2925,1624,1464,1425$.

TLC (1:1) hexane/EtOAc, permanganate, $\mathrm{R}_{f}=0.33$.

HRMS calculated for $\left[\mathrm{C}_{17} \mathrm{H}_{35} \mathrm{NO}_{2} \mathrm{H}\right]^{+}$, requires $m / z=286.2746$, found $m / z=286.2748$ (ESI).

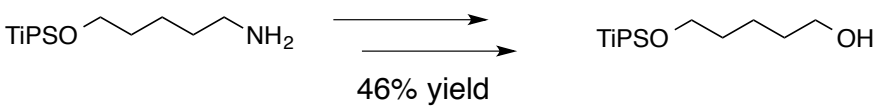

14

15

Synthesis of alcohol 15 The standard procedure was followed beginning with amine 14 (332 $\mathrm{mg}, 1.24 \mathrm{mmol}, 1$ equiv). The crude product was purified by column chromatography $\left(3: 1\right.$ hexane $/ \mathrm{Et}_{2} \mathrm{O}, 40 \mathrm{~mL}$ silica gel) to yield alcohol 15 (148 mg, $0.569 \mathrm{mmol}, 46 \%$ yield). Compound analysis is consistent with published data. ${ }^{6}$

${ }^{1} \mathrm{H}$ NMR $\left(\mathrm{CDCl}_{3}, 200 \mathrm{MHz}\right) \delta 3.73-3.60\left(\mathrm{~m}, 4 \mathrm{H}, 2 \times \mathrm{O}-\mathrm{CH}_{2}\right), 1.68-1.30\left(\mathrm{~m}, 9 \mathrm{H},\left(\mathrm{CH}_{2}\right)_{3}, 3 \mathrm{x}\right.$ iPr), 1.10-1.04 (brs, $18 \mathrm{H}, 3 \mathrm{x}$ iPr).

TLC (3:1) hexane/ $/ \mathrm{Et}_{2} \mathrm{O}$, permanganate, $\mathrm{R}_{f}=0.28$.<smiles>NCCCOc1ccc([N+](=O)[O-])cc1</smiles>

16

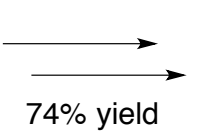

$74 \%$ yield<smiles>O=[N+]([O-])c1ccc(OCCCO)cc1</smiles>

17

Synthesis of alcohol 17 The standard procedure was followed beginning with amine $\mathbf{1 6}$ (308 $\mathrm{mg}, 1.57 \mathrm{mmol}, 1$ equiv). The crude product was purified by column chromatography (2:1 EtOAc/hexane, $30 \mathrm{~mL}$ silica gel) to yield alcohol 17 (230 mg, $1.16 \mathrm{mmol}, 74 \%$ yield). Compound analysis is consistent with published data. ${ }^{7}$

${ }^{1} \mathbf{H}$ NMR $\left(\mathrm{CDCl}_{3}, 200 \mathrm{MHz}\right) \delta 8.20(\mathrm{~d}, J=9.2 \mathrm{~Hz}, 2 \mathrm{H}, \mathrm{Ar}), 6.97(\mathrm{~d}, J=9.3 \mathrm{~Hz}, 2 \mathrm{H}, \mathrm{Ar}), 4.22(\mathrm{t}, J=6.2 \mathrm{~Hz}$, $\left.2 \mathrm{H}, \mathrm{Ar}-\mathrm{O}-\mathrm{CH}_{2}\right), 3.88\left(\mathrm{t}, J=6.0 \mathrm{~Hz}, 2 \mathrm{H}, \mathrm{O}-\mathrm{CH}_{2}\right), 2.09$ (pent, $J=6.0 \mathrm{~Hz}, 2 \mathrm{H}, \mathrm{C}-\mathrm{CH}_{2}-\mathrm{C}$ ), $1.52(\mathrm{bs}, 1 \mathrm{H}, \mathrm{OH})$.

TLC (2:1) EtOAc/hexane, permanganate, $\mathrm{R}_{f}=0.34$.

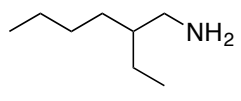

18

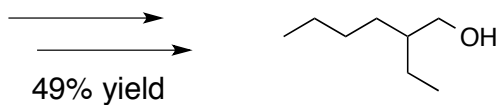

19

Synthesis of alcohol 19 The standard procedure was followed beginning with 2-ethylhexylamine (18,500 $\mathrm{mg}$, $3.87 \mathrm{mmol}, 1$ equiv). To minimize loss of material into the aqueous washes, the workup of the nitrosylation step was

\footnotetext{
${ }^{6}$ Compound data from: Crimmins, M. T.; Vanier, G. S. Org. Lett. 2006, 8, 2887-2890.

${ }^{7}$ Compound data from: Steel W. H.; Damkaci, F.; Nolan, R.; Walker, R. A. J. Am. Chem. Soc. 2002, 124, 48244831 .
} 
modified to use 1:1 mixtures of brine and 10\% sodium carbonate for the base washes and a reduced amount of potassium phosphate monobasic for the final wash. Similarly, the workup of the hydrolysis step was modified to use a dilution of $50 \%$ brine rather than water. The crude product was purified by column chromatography $(3: 2$ hexane/ $\mathrm{Et}_{2} \mathrm{O}, 50 \mathrm{~mL}$ silica gel) to yield alcohol 19 (247 mg, $1.89 \mathrm{mmol}, 49 \%$ yield). Prolonged exposure to high vacuum was avoided due to the product's moderate volatility $\left(\mathrm{BP}=180^{\circ} \mathrm{C}\right)$. Compound analysis is consistent with data from commercially available material. ${ }^{8}$

${ }^{1} \mathbf{H}$ NMR $\left(\mathrm{CDCl}_{3}, 600 \mathrm{MHz}\right) \delta 3.55\left(\mathrm{~d}, J=5.4 \mathrm{~Hz}, 2 \mathrm{H}, \mathrm{O}-\mathrm{CH}_{2}\right), 1.44-1.24\left(\mathrm{~m}, 9 \mathrm{H}, \mathrm{CH}, 4 \times \mathrm{CH}_{2}\right), 0.90(\mathrm{t}, J=$ $\left.7.4 \mathrm{~Hz}, 6 \mathrm{H}, 2 \times \mathrm{CH}_{3}\right)$.

TLC (3:2) hexane/ether, permanganate, $\mathrm{R}_{f}=0.46$.

${ }^{8}$ Compound data from: www.sigmaaldrich.com 


\subsection{Synthesis of Amine Substrates from the Substrate Scope Table}

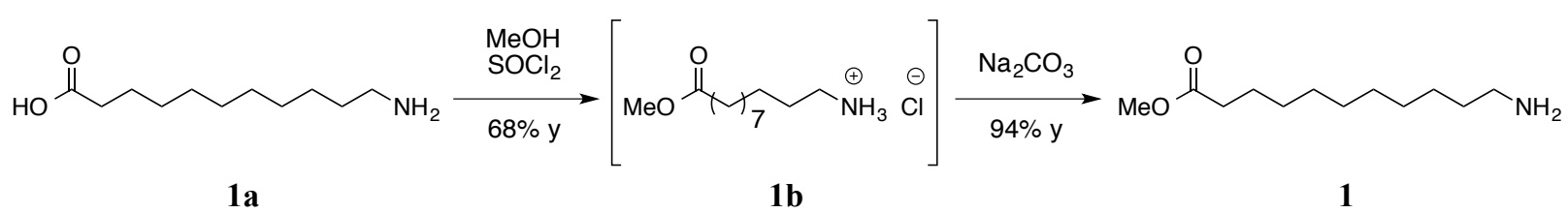

Synthesis of ester hydrochloride 1b Into a flask were added 11-aminoundecanoic acid (1a, 10.0 g, $49.7 \mathrm{mmol}$, 1 equiv) and $\mathrm{MeOH}(50 \mathrm{~mL}, 1 \mathrm{M})$. The mixture was cooled in an ice bath $\left(0^{\circ} \mathrm{C}\right)$. Thionyl chloride $(7.25 \mathrm{~mL}, 99.4$ mmol, 2 equiv) was added dropwise. The mixture was heated to reflux $(3 \mathrm{~h})$, allowed to cool to ambient temperature, and diluted with EtOAc $(100 \mathrm{~mL}$, producing white precipitate). Solids were isolated by vacuum filtration and dried under vacuum to yield pure ester hydrochloride $\mathbf{1 b}(8.45 \mathrm{~g}, 33.6 \mathrm{mmol}, 68 \%$ yield, white solid), which was used directly in the next step.

Synthesis of ester 1 Into a flask were added ester hydrochloride $\mathbf{1 b}(4.00 \mathrm{~g}, 15.9 \mathrm{mmol}, 1$ equiv) and sodium carbonate $(10 \%$ aqueous, $60 \mathrm{~mL})$. The mixture was stirred $(5 \mathrm{~min})$ and extracted with EtOAc $(50+40+20 \mathrm{~mL})$. The combined organic phase was dried with sodium sulfate. Volatiles were removed under reduced pressure to yield pure ester 1 (3.20 g, $14.9 \mathrm{mmol}, 94 \%$ yield, translucent oil). Compound analysis is consistent with published data. ${ }^{9}$

${ }^{1} \mathbf{H}$ NMR $\left(\mathrm{CDCl}_{3}, 200 \mathrm{MHz}\right) \delta 3.67(\mathrm{~s}, 3 \mathrm{H}, \mathrm{OMe}), 2.68\left(\mathrm{t}, J=6.6 \mathrm{~Hz}, 2 \mathrm{H}, \mathrm{N}-\mathrm{CH}_{2}\right), 2.31(\mathrm{t}, J=7.5 \mathrm{~Hz}, 2 \mathrm{H}$, $\left.\mathrm{O}=\mathrm{C}-\mathrm{CH}_{2}\right), 1.71-1.52\left(\mathrm{~m}, 2 \mathrm{H}, \mathrm{CH}_{2}\right), 1.50-1.07\left(\mathrm{~m}, 16 \mathrm{H}, \mathrm{NH}_{2},\left(\mathrm{CH}_{2}\right)_{7}\right)$.

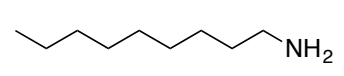

8

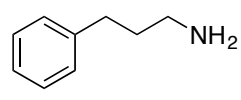

10

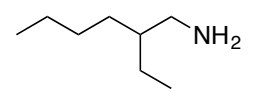

18

Nonylamine (8), 3-phenylpropylamine (10), and 2-ethylhexylamine (18) are commercially available.

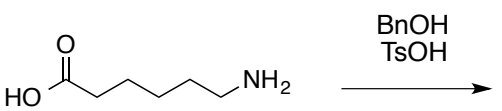

$10 \mathbf{a}$

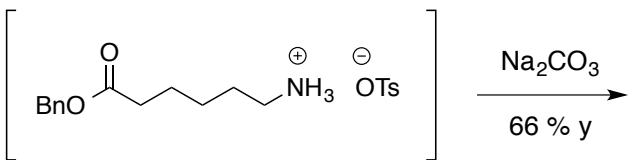

10b

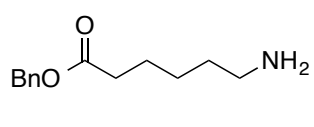

10

Synthesis of ester 10 Into a flask were added 6-aminohexanoic acid (10a, $1.32 \mathrm{~g}, 10.1 \mathrm{mmol}, 1$ equiv), toluene ( $25 \mathrm{~mL}, 0.4 \mathrm{M})$, benzyl alcohol $(3.67 \mathrm{~mL}, 35.3 \mathrm{mmol}, 3.5$ equiv), and p-toluenesulfonic acid hydrate $(3.00 \mathrm{~g}, 15.2$ mmol, 1.5 equiv). Using a Dean-Stark apparatus the mixture was heated to reflux $(18 \mathrm{~h})$ and allowed to cool to ambient temperature (producing white precipitate). Solids were isolated by vacuum filtration, washed with ether, and dried under vacuum to yield curde tosylate salt $10 \mathrm{~b}$ ( $3.00 \mathrm{~g}, 7.63 \mathrm{mmol}, 76 \%$ crude yield), which was used directly in the next step. Compound analysis is consistent with published data. ${ }^{10}$

${ }^{1} \mathbf{H}$ NMR $\left(\mathrm{D}_{2} \mathrm{O}, 200 \mathrm{MHz}\right) \delta 7.72(\mathrm{~d}, J=8.0 \mathrm{~Hz}, 2 \mathrm{H}, \mathrm{Ts}), 7.49-7.44(\mathrm{~m}, 5 \mathrm{H}, \mathrm{Bn}), 7.39(\mathrm{~d}, J=8.0 \mathrm{~Hz}, 2 \mathrm{H}, \mathrm{Ts})$, $5.20(\mathrm{~s}, 2 \mathrm{H}, \mathrm{Bn}), 2.97\left(\mathrm{t}, J=6.9 \mathrm{~Hz}, 2 \mathrm{H}, \mathrm{CH}_{2}-\mathrm{N}\right), 2.49\left(\mathrm{t}, J=7.5 \mathrm{~Hz}, 2 \mathrm{H}, \mathrm{O}=\mathrm{C}-\mathrm{CH}_{2}\right), 1.66$ (pent, $J=7.4 \mathrm{~Hz}, 4 \mathrm{H}, 2$ x $\left.\mathrm{CH}_{2}\right), 1.50-1.28\left(\mathrm{~m}, 2 \mathrm{H}, \mathrm{CH}_{2}\right)$.

Into an extraction funnel were added one third of crude tosylate salt $10 \mathrm{~b}(1.00 \mathrm{~g}, 2.54 \mathrm{mmol})$ and sodium carbonate $(10 \%$ aqueous, $50 \mathrm{~mL})$. The mixture was mixed until homogeneous, extracted with EtOAc $(3 \times 30 \mathrm{~mL})$, and dried with sodium sulfate. Volatiles were removed under reduced pressure to yield ester $\mathbf{1 0}$ (491 $\mathrm{mg}, 2.22$ $\mathrm{mmol}, 66 \%$ yield from acid 10a).

\footnotetext{
${ }^{9}$ Compound data from: Moon, K.-S.; Lee, E.; Lim, Y.-B.; Lee, M. Chem. Commun. 2008, 4001-4003.

${ }^{10}$ Compound data from: Machida, S.; Kato, N.; Harada, K.; Ohkanda, J. J. Am. Chem. Soc. 2010, 133, $985-963$
} 
${ }^{1} \mathbf{H}$ NMR $\left(\mathrm{CDCl}_{3}, 200 \mathrm{MHz}\right) \delta 7.46-7.29(\mathrm{~m}, 5 \mathrm{H}, \mathrm{Ar}), 5.11\left(\mathrm{~s}, 2 \mathrm{H}, \mathrm{O}-\mathrm{CH}_{2}\right), 2.67\left(\mathrm{t}, J=6.7 \mathrm{~Hz}, 2 \mathrm{H}, \mathrm{CH}_{2}-\mathrm{N}\right)$, 2.37, (t, $\left.J=7.4 \mathrm{~Hz}, 2 \mathrm{H}, \mathrm{CH}_{2}-\mathrm{C}=\mathrm{O}\right), 1.67$ (pent, $\left.J=7.3 \mathrm{~Hz}, \mathrm{CH}_{2}-\mathrm{C}-\mathrm{N}\right), 1.55-1.25\left(\mathrm{~m}, 4 \mathrm{H}_{1}\left(\mathrm{CH}_{2}\right)_{2}\right), 1.25(\mathrm{brs}, 2 \mathrm{H}$, $\mathrm{NH}_{2}$ ).

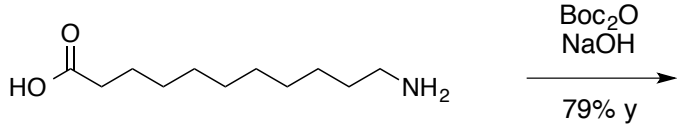

$12 \mathrm{a}$

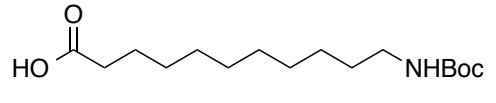

$12 b$

Synthesis of carbamate 12b Into a flask were added 11-aminoundecanoic acid (12a, $1.00 \mathrm{~g}, 4.97 \mathrm{mmol}, 1$ equiv) and dioxane $(6.2 \mathrm{~mL}, 0.8 \mathrm{M})$. Sodium hydroxide $(0.19 \mathrm{~g}, 5.0 \mathrm{mmol}, 1$ equiv) was dissolved in water $(10 \mathrm{~mL}$, $0.8 \mathrm{M})$ and added to the dioxane solution. The mixture was cooled in an ice bath $\left(0{ }^{\circ} \mathrm{C}, 20 \mathrm{~min}\right)$. Di-tert-butyl dicarbonate (1.19 g, $5.46 \mathrm{mmol}, 1.1$ equiv) was dissolved in dioxane $(6.2 \mathrm{~mL})$ and added. The suspension was stirred vigorously and allowed to gradually warm to ambient temperature $(26 \mathrm{~h})$. Volatiles were removed under reduced pressure. The residue was dissolved in a mixture of water $(40 \mathrm{~mL})$ and saturated sodium bicarbonate $(10$ $\mathrm{mL})$, washed with EtOAc $(2 \times 30 \mathrm{~mL})$, acidified with sodium bisulfate (1 M, $30 \mathrm{~mL}$, to pH 2), extracted with EtOAc $(40 \mathrm{~mL}$ plus $2 \times 30 \mathrm{~mL})$, and dried with sodium sulfate. Volatiles were removed under reduced pressure to yield pure carbamate $\mathbf{1 2 b}$ (1.16 g, $3.85 \mathrm{mmol}, 79 \%$ yield), which was used directly in the next step. Compound analysis is consistent with published data. ${ }^{11}$

${ }^{1} \mathbf{H}$ NMR $\left(\mathrm{CDCl}_{3}, 200 \mathrm{MHz}\right) \delta 4.51$ (brs, $\left.1 \mathrm{H}, \mathrm{NH}\right), 3.10$ (q, $\left.J=6.6 \mathrm{~Hz} 2 \mathrm{H}, \mathrm{N}-\mathrm{CH}_{2}\right), 2.35(\mathrm{t}, J=7.3 \mathrm{~Hz}, 2 \mathrm{H}$, $\left.\mathrm{O}=\mathrm{C}-\mathrm{CH}_{2}\right), 1.69-1.55\left(\mathrm{~m}, 2 \mathrm{H}, \mathrm{N}-\mathrm{C}-\mathrm{CH}_{2}\right), 1.44(\mathrm{~s}, 9 \mathrm{H}, \mathrm{Boc}), 1.38-1.22\left(\mathrm{~m}, 14 \mathrm{H},\left(\mathrm{CH}_{2}\right)_{7}\right)$.

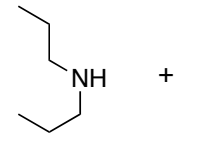

$12 \mathrm{c}$

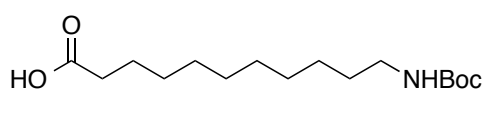

$12 b$

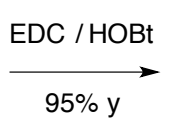

Synthesis of amide 12d Into a flask were added carbamate 12b (1.16 g, 3.85 mmol, 1 equiv), DCM (3.9 mL, 1 M), dipropylamine (12c, $0.56 \mathrm{~mL}, 7.74 \mathrm{mmol}, 2$ equiv), EDC hydrochloride (1.48 g, $7.73 \mathrm{mmol}, 2$ equiv), and HOBt hydrate $(0.59 \mathrm{~g}, 3.87 \mathrm{mmol}, 1$ equiv). The mixture was stirred $(24 \mathrm{~h})$, diluted with sodium bisulfate $(1 \mathrm{M}, 50 \mathrm{~mL})$, and extracted with ether $(2 \times 50 \mathrm{~mL}+25 \mathrm{~mL})$. The combined organic phases were washed with sodium carbonate $(10 \%, 70 \mathrm{~mL})$ and dried with sodium sulfate. Volatiles were removed under reduced pressure to yield pure amide 12d (1.42 g, $3.69 \mathrm{mmol}, 95 \%$ yield), which was used directly in the next step.

${ }^{1} \mathbf{H}$ NMR $\left(\mathrm{CDCl}_{3}, 200 \mathrm{MHz}\right) \delta 4.50$ (brs, $\left.1 \mathrm{H}, \mathrm{NH}\right), 3.32-3.13\left(\mathrm{~m}, 4 \mathrm{H}, \mathrm{CH}_{2}-\mathrm{N}-\mathrm{CH}_{2}\right), 3.10(\mathrm{q}, J=6.6 \mathrm{~Hz}, 2 \mathrm{H}$, $\left.\mathrm{O}=\mathrm{C}-\mathrm{N}-\mathrm{CH}_{2}\right), 2.28\left(\mathrm{t}, J=7.5 \mathrm{~Hz}, 2 \mathrm{H}, \mathrm{O}=\mathrm{C}-\mathrm{CH}_{2}\right), 1.68-1.40\left(\mathrm{~m}, 6 \mathrm{H}, 3 \mathrm{x} \mathrm{N}-\mathrm{C}-\mathrm{CH}_{2}\right), 1.44(\mathrm{~s}, 9 \mathrm{H}, \mathrm{Boc}), 1.38-1.22$ $\left(\mathrm{m}, 14 \mathrm{H},\left(\mathrm{CH}_{2}\right)_{7}\right), 0.90\left(\mathrm{q}, J=7.5 \mathrm{~Hz}, 6 \mathrm{H}, 2 \times \mathrm{CH}_{3}\right)$.

${ }^{13} \mathrm{C} \mathrm{NMR}\left(\mathrm{CDCl}_{3}, 50 \mathrm{MHz}\right) \delta 172.13,155.61,77.97,49.09,46.92,40.09,32.56,29.57,28.93,27.92,26.29$, 24.99, 21.82, 20.45, 10.86, 10.71.

IR (thin film, $\mathrm{cm}^{-1}$ ) 3335, 2927, 1698, 1631, 1176.

TLC (1:1) hexane/EtOAc, permanganate, $\mathrm{R}_{f}=0.57$.

HRMS calculated for $\left[\mathrm{C}_{22} \mathrm{H}_{44} \mathrm{~N}_{2} \mathrm{O}_{3} \mathrm{H}\right]^{+}$, requires $m / z=385.3430$, found $m / z=385.3429$ (ESI).

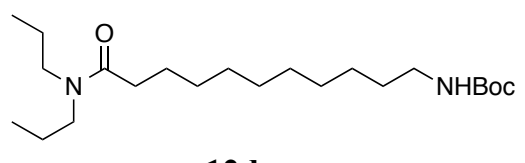

12d

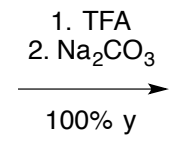

$00 \%$ y

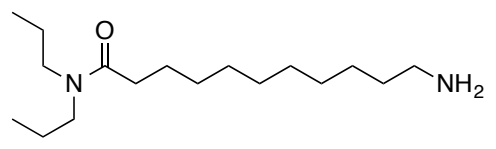

12

Synthesis of amine 12 Into a flask were added carbamate 12d (1.42 g, 3.69 mmol, 1 equiv), DCM (21 mL, 0.2 $\mathrm{M})$, and TFA $(8 \mathrm{~mL})$. The mixture was stirred $(2 \mathrm{~h})$, and volatiles were removed under reduced pressure (toluene

\footnotetext{
${ }^{11}$ Compound data from: Amara, N.; Mashiach, R.; Amar, D.; Krief, P.; Spieser, S. A. H.; Bottomley, M. J.; Aharoni, A.; Meijler, M. M. J. Am. Chem. Soc. 2009, 131, 10610-10619.
} 
azeotrope). The crude oil was diluted with a mixture of sodium carbonate $(10 \%$ aqueous, $30 \mathrm{~mL})$ and brine $(25 \mathrm{~mL})$, extracted with EtOAc $(30 \mathrm{~mL}+2 \times 20 \mathrm{~mL})$, and dried with sodium sulfate. Volatiles were removed under reduced pressure to yield free amine $\mathbf{1 2}(1.06 \mathrm{~g}, 3.69 \mathrm{mmol}, 100 \%$ yield $)$.

${ }^{1} \mathbf{H}$ NMR $\left(\mathrm{CDCl}_{3}, 200 \mathrm{MHz}\right) \delta 4.50$ (brs, $\left.2 \mathrm{H}, \mathrm{NH}_{2}\right), 3.32-3.13\left(\mathrm{~m}, 4 \mathrm{H}, \mathrm{CH}_{2}-\mathrm{N}-\mathrm{CH}_{2}\right), 2.77(\mathrm{t}, J=7.2 \mathrm{~Hz}, 2 \mathrm{H}$, $\left.\mathrm{CH}_{2}-\mathrm{N}\right), 2.28\left(\mathrm{t}, J=7.5 \mathrm{~Hz}, 2 \mathrm{H}, \mathrm{O}=\mathrm{C}-\mathrm{CH}_{2}\right), 1.68-1.43\left(\mathrm{~m}, 4 \mathrm{H}, 2 \times \mathrm{CH}_{2}-\mathrm{C}-\mathrm{N}\right), 1.43-1.20\left(\mathrm{~m}, 14 \mathrm{H},\left(\mathrm{CH}_{2}\right)_{7}\right), 0.90$ (q, $J=7.5 \mathrm{~Hz}, 4 \mathrm{H}, 2 \times \mathrm{CH}_{3}$ ).

${ }^{13} \mathbf{C}$ NMR $\left(\mathrm{CDCl}_{3}, 50 \mathrm{MHz}\right) \delta 171.51,48.56,46.38,40.71,31.98,28.41,25.82,24.47,21.30,19.92,10.26$, 10.15 .

IR (thin film, $\mathrm{cm}^{-1}$ ) 2927, 1641, 1464, 1427.

HRMS calculated for $\left[\mathrm{C}_{17} \mathrm{H}_{36} \mathrm{~N}_{2} \mathrm{OH}\right]^{+}$, requires $m / z=285.2906$, found $m / z=258.2909$ (ESI).

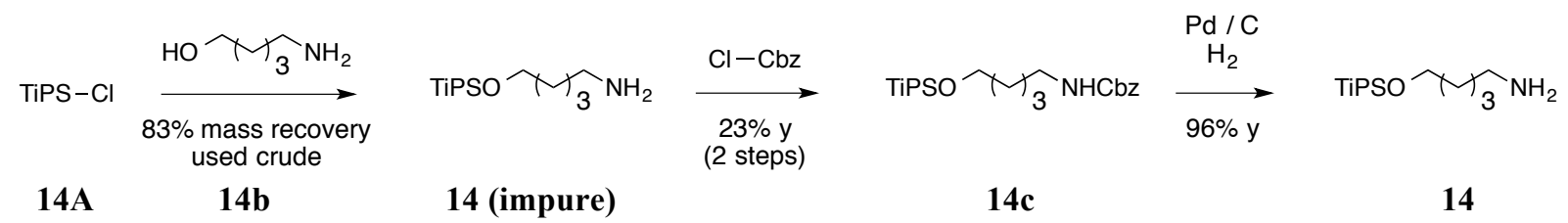

Synthesis of silyl ether 14 Into a flask were added 5-amino-1-pentanol (14b, $1.00 \mathrm{~g}, 9.69 \mathrm{mmol}, 1$ equiv), DCM (39 mL, $0.25 \mathrm{M}$ ), and imidazole $(956 \mathrm{mg}, 14.1 \mathrm{mmol}, 1.5$ equiv). Triisopropylsilyl chloride was added dropwise to the mixture $(5 \mathrm{~min})$. The mixture was stirred at ambient temperature $(140 \mathrm{~h}$, evaporating most of the DCM, shorter reaction time is likely sufficient), diluted with a mixture of brine and water $(1: 1,100 \mathrm{~mL})$, and extracted with ether $(2 \times 50 \mathrm{~mL}+30 \mathrm{~mL})$. The combined organic phase was washed with sodium carbonate $(5 \%$ aqueous, $50 \mathrm{~mL}$ ) and dried with sodium sulfate. Volatiles were removed under reduced pressure to yield impure silyl ether 14 (2.08 g, 83\% mass recovery), a fraction of which was used crude for the next step. (Since amine $\mathbf{1 4}$ is difficult to purify directly, it was protected as a benzyl carbamate, purified, and deprotected.)

Synthesis of benzyl carbamate 14c Into a flame dried flask were added $86 \%$ of crude silyl ether 14 (6.92 mmol, 1 equiv), DCM (43 mL, $0.2 \mathrm{M})$, and TEA ( $2.12 \mathrm{~mL}, 15.2 \mathrm{mmol}, 2.2$ equiv). The flask was capped with a rubber septum and vented through a syringe packed with anhydrous calcium sulfate. Benzyl chloroformate $(0.99$ $\mathrm{mL}, 6.96 \mathrm{mmol}, 1$ equiv) was added dropwise (over $5 \mathrm{~min})$. The mixture was stirred $(18 \mathrm{~h}$ ), the organic layer was washed with sodium phosphate ( $1 \mathrm{M}$ aqueous, $\mathrm{pH} 5,50 \mathrm{~mL})$. The aqueous phase was further extracted with DCM (2 x $50 \mathrm{~mL}$ ), and the combined organic phases were washed with sodium bicarbonate (saturated aqueous, $65 \mathrm{~mL}$ ) and dried with sodium sulfate. Volatiles were removed under reduced pressure, and the crude material was purified by column chromatography $\left(3: 1\right.$ hexane/Et ${ }_{2} \mathrm{O}, 100 \mathrm{~mL}$ silica gel) to yield pure carbamate $14 \mathrm{c}(640 \mathrm{mg}, 1.63 \mathrm{mmol}$, $23 \%$ yield, plus additional impure material).

${ }^{1} \mathbf{H}$ NMR $\left(\mathrm{CDCl}_{3}, 200 \mathrm{MHz}\right) \delta 7.41-7.29(\mathrm{~m}, 5 \mathrm{H}, \mathrm{Ar}), 5.10$ (s, 2H, Bn), 4.72 (brs, $\left.1 \mathrm{H}, \mathrm{NH}\right), 3.67$ (t, $J=6.1 \mathrm{~Hz}$, $\left.2 \mathrm{H}, \mathrm{O}-\mathrm{CH}_{2}\right), 3.20\left(\mathrm{q}, J=6.3 \mathrm{~Hz}, 2 \mathrm{H}, \mathrm{N}-\mathrm{CH}_{2}\right), 1.64-1.22\left(\mathrm{~m}, 9 \mathrm{H},\left(\mathrm{CH}_{2}\right)_{3}, 3 \mathrm{x}\right.$ iPr), $1.10-1.00$ (brs, $18 \mathrm{H}, 3 \mathrm{x}$ iPr).

${ }^{13} \mathbf{C}$ NMR $\left(\mathrm{CDCl}_{3}, 50 \mathrm{MHz}\right) \delta 156.37,136.65,128.46,128.04,66.53,63.12,41.08,32.53,29.74,23.03,17.98$, 11.96.

IR (neat, $\mathrm{cm}^{-1}$ ) 2941, 2864, 1701, 1520, 1463, 1102, 908, 882.

TLC (3:1) hexane/ $\mathrm{Et}_{2} \mathrm{O}$, permanganate, $\mathrm{R}_{f}=0.51$.

HRMS calculated for $\left[\mathrm{C}_{22} \mathrm{H}_{39} \mathrm{NO}_{3} \mathrm{SiH}\right]^{+}$, requires $m / z=394.2777$, found $m / z=394.2772$ (ESI).

Synthesis of amine 14 Into a flask were added carbamate $14 \mathrm{c}(585 \mathrm{mg}, 1.49 \mathrm{mmol}, 1$ equiv) and $\mathrm{MeOH}(5$ $\mathrm{mL})$. Into another flask was added palladium on carbon $(10 \% \mathrm{Pd}, 75 \mathrm{mg}, 50 \mathrm{mg} / \mathrm{mmol}$ substrate). The palladiumcontaining flask was capped with a septum and flushed with nitrogen. $\mathrm{MeOH}(2.5 \mathrm{~mL})$ was added by syringe, the flask was uncapped, and the slurry was transferred to the substrate-containing flask (final concentration $=0.2 \mathrm{M}$ ). The mixture was capped and flushed with nitrogen $(5 \mathrm{~min})$. A hydrogen-filled balloon was attached such that its needle remained submerged, the flask was flushed briefly with hydrogen $(30 \mathrm{sec})$, and the reaction was stirred at ambient temperature under the balloon's pressure $(4 \mathrm{~h})$. The balloon was removed, and the flask was flushed with nitrogen. The mixture was filtered through Celite ( $\mathrm{MeOH}$ chase), and volatiles were removed under reduced pressure to yield pure amine 14 (373 $\mathrm{mg}, 1.44 \mathrm{mmol}, 96 \%$ yield). 
${ }^{1} \mathbf{H}$ NMR $\left(\mathrm{CDCl}_{3}, 200 \mathrm{MHz}\right) \delta 3.68\left(\mathrm{t}, J=6.3 \mathrm{~Hz}, 2 \mathrm{H}, \mathrm{O}-\mathrm{CH}_{2}\right), 2.70\left(\mathrm{t}, J=6.6 \mathrm{~Hz}, 2 \mathrm{H}, \mathrm{N}-\mathrm{CH}_{2}\right), 1.78-1.64$ (brs, $\left.2 \mathrm{H}, \mathrm{NH}_{2}\right), 1.64-1.26$ (m, 9H, $\left(\mathrm{CH}_{2}\right)_{3}, 3 \mathrm{x}$ iPr), 1.11-0.99 (brs, $18 \mathrm{H}, 3 \mathrm{x}$ iPr).

${ }^{13} \mathbf{C}$ NMR $\left(\mathrm{CDCl}_{3}, 50 \mathrm{MHz}\right) \delta 63.22,41.86,33.01,32.74,23.06,17.97,11.95$.

IR (neat, $\mathrm{cm}^{-1}$ ) 2940, 2865, 1463, 1103, 882.

HRMS calculated for $\left[\mathrm{C}_{14} \mathrm{H}_{33} \mathrm{NOSiH}\right]^{+}$, requires $\mathrm{m} / z=260.2410$, found $m / z=260.2411$ (ESI).

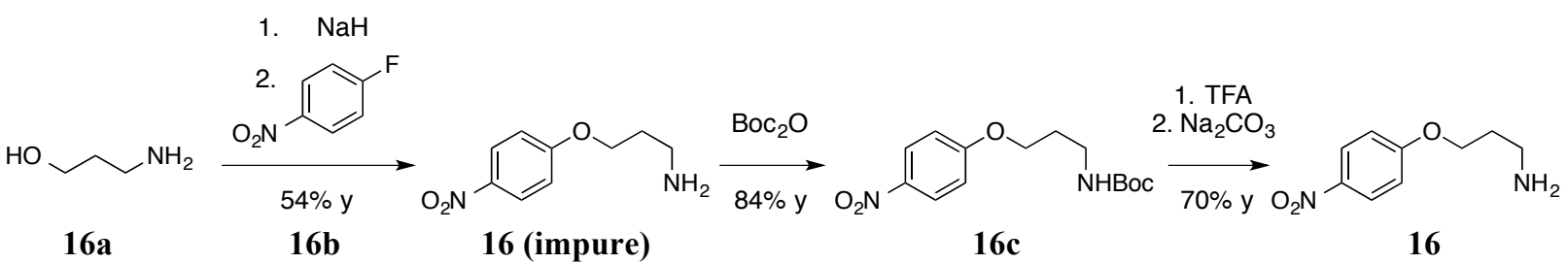

Synthesis of ether 16 Into a flame-dried flask was added sodium hydride ( $320 \mathrm{mg}, 13.3 \mathrm{mmol}, 1$ equiv). The flask was capped with a rubber septum, maintained under a nitrogen atmosphere, and cooled in an ice bath $\left(0^{\circ} \mathrm{C}\right)$. DMF (8.8 mL, 1.5 M) was added. 3-Aminopropanol (16a, $1.00 \mathrm{~g}, 13.3 \mathrm{mmol}, 1$ equiv) was dissolved in DMF (4.4 $\mathrm{mL}$ ) and added dropwise to the reaction (over $5 \mathrm{~min}$, producing gas). The mixture was stirred (additional $5 \mathrm{~min}$ ). 4Fluoronitrobenzene (16b, $1.88 \mathrm{~g}, 13.3 \mathrm{mmol}, 1$ equiv) was dissolved in DMF (4.4 mL) and added dropwise to the reaction (over $5 \mathrm{~min}$, becoming bright orange, final concentration $=0.8 \mathrm{M})$. The mixture was stirred $(10 \mathrm{~min}$ ), allowed to warm to ambient temperature, stirred (additional $3 \mathrm{~h}$ ), diluted with water $(150 \mathrm{~mL}$ ), acidified with sodium bisulfate ( $1 \mathrm{M}$ aqueous, $40 \mathrm{~mL}$, to $\mathrm{pH} 2)$, washed with ether $(2 \times 50 \mathrm{~mL})$, basified with sodium hydroxide $(1 \mathrm{M}$ aqueous, $20 \mathrm{~mL}$, plus $600 \mathrm{mg}$ solid), extracted with ether $(3 \times 50 \mathrm{~mL})$, and dried with sodium sulfate. Volatiles were removed under reduced pressure to yield crude ether $\mathbf{1 6}(1.42 \mathrm{~g}, 7.24 \mathrm{mmol}, 54 \%$ crude yield), which was used directly in the next step. (Since amine $\mathbf{1 6}$ is difficult to purify, it was protected as a carbamate, purified, and deprotected.)

Synthesis of carbamate 16c Into a flask were added crude amine 16 (1.22 g, $6.19 \mathrm{mmol}, 1$ equiv), dioxane (8.2 $\mathrm{mL})$, water $(8.2 \mathrm{~mL})$, and sodium hydroxide $\left(248 \mathrm{mg}, 6.19 \mathrm{mmol}, 1\right.$ equiv). The flask was stirred in an ice bath $\left(0^{\circ}\right.$ C). Di-tert-butyl dicarbonate $(1.49 \mathrm{~g}, 6.81 \mathrm{mmol}, 1.1$ equiv) was dissolved in dioxane $(8.2 \mathrm{~mL})$ and added to the reaction (final concentration $=0.3 \mathrm{M}$ ). The mixture was stirred and allowed to gradually warm to ambient temperature $(19 \mathrm{~h})$. Volatiles were removed under reduced pressure. The residue was dissolved in sodium carbonate $(10 \%$ aqueous, $100 \mathrm{~mL})$, extracted with EtOAc $(3 \times 50 \mathrm{~mL})$, washed with potassium phosphate monobasic $(1 \mathrm{M}$ aqueous, $\mathrm{pH} 5,50 \mathrm{~mL}$ ), and dried with sodium sulfate. Volatiles were removed under reduced pressure, and the crude material was purified by column chromatography $\left(1: 1\right.$ hexane/Et $\mathrm{t}_{2} \mathrm{O}, 40 \mathrm{~mL}$ silica gel) to yield pure carbamate $16 \mathrm{c}(1.54 \mathrm{~g}, 5.20 \mathrm{mmol}, 84 \%$ yield $)$.

${ }^{1} \mathrm{H}$ NMR $\left(\mathrm{CDCl}_{3}, 200 \mathrm{MHz}\right) \delta 8.20(\mathrm{~d}, J=9.2 \mathrm{~Hz}, 2 \mathrm{H}, \mathrm{Ar}), 6.96(\mathrm{~d}, J=9.2 \mathrm{~Hz}, 2 \mathrm{H}, \mathrm{Ar}), 4.67$ (brs, $\left.1 \mathrm{H}, \mathrm{NH}\right)$, $4.11\left(\mathrm{t}, J=6.2 \mathrm{~Hz}, 2 \mathrm{H}, \mathrm{O}-\mathrm{CH}_{2}\right), 3.34$ (q, $\left.J=6.5 \mathrm{~Hz}, 2 \mathrm{H}, \mathrm{N}-\mathrm{CH}_{2}\right), 1.97$ (pent, $\left.J=6.5 \mathrm{~Hz}, 2 \mathrm{H}, \mathrm{CH}_{2}\right), 1.44(\mathrm{~s}, 9 \mathrm{H}$, $\left.\left(\mathrm{CH}_{3}\right)_{3}\right)$.

TLC (1:1) hexane/EtOAc, permanganate, $\mathrm{R}_{f}=0.28$.

Synthesis of amine 16 Into a flask were added carbamate $16 \mathrm{c}(1.54 \mathrm{~g}, 5.20 \mathrm{mmol}, 1$ equiv), DCM $(29 \mathrm{~mL})$, and TFA $(5 \mathrm{~mL})$ and the mixture (final concentration $=0.2 \mathrm{M}, 6: 1)$ was stirred $(1.5 \mathrm{~h})$. Volatiles were removed under reduced pressure ( $3 \mathrm{x}$ toluene azeotrope). The residue was dissolved in sodium carbonate (saturated aqueous, 100 $\mathrm{mL})$ and brine $(20 \mathrm{~mL})$, extracted with ether $(3 \times 50 \mathrm{~mL})$, and dried with sodium sulfate. Volatiles were removed under reduced pressure to yield pure amine 16 (719 mg, $3.67 \mathrm{mmol}, 70 \%$ yield). Compound analysis is consistent with published data. ${ }^{12}$

${ }^{1} \mathbf{H}$ NMR $\left(\mathrm{CDCl}_{3}, 200 \mathrm{MHz}\right) \delta 8.20(\mathrm{~d}, J=9.2 \mathrm{~Hz}, 2 \mathrm{H}, \mathrm{Ar}), 6.96(\mathrm{~d}, J=9.2 \mathrm{~Hz}, 2 \mathrm{H}, \mathrm{Ar}), 4.16(\mathrm{t}, J=6.2 \mathrm{~Hz}$, $2 \mathrm{H}, \mathrm{O}-\mathrm{CH}_{2}$ ), $2.93\left(\mathrm{q}, J=6.3 \mathrm{~Hz}, 2 \mathrm{H}, \mathrm{N}-\mathrm{CH}_{2}\right.$ ), 1.97 (pent, $J=6.5 \mathrm{~Hz}, 2 \mathrm{H}, \mathrm{CH}_{2}$ ), 1.22 (brs, $2 \mathrm{H}, \mathrm{NH}_{2}$ ).

\footnotetext{
${ }^{12}$ Compound data from: Nakhara, S.; Minami, S. Liquid Crystal Aligning Agent. PCT Int. Appl., 2010-053128, May, 14, 2010.
} 


\subsection{Formation of Imidyol Trifluoroacetate 28}

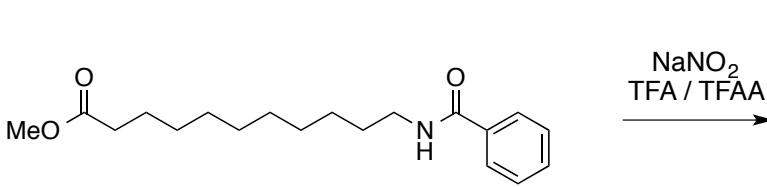

27

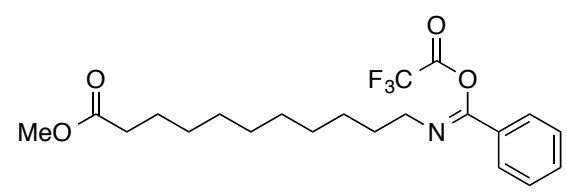

28

Formation of imidoyl trifluoroacetate 28 The standard nitrosylation procedure (see section 2.9) was followed using benzamide 27 and a solvent mixture of 5:1:1 EtOAc / trifluoroacetic acid / trifluoroacetic anhydride. Imidoyl trifluoroacetate 28 was recovered as the major product ( $>95 \%$ conversion). Since the product is not fully stable to silica gel, it was analyzed crude. Similar imidoyl trifluoroacetetes have been previously reported. ${ }^{13}$

${ }^{1} \mathbf{H}$ NMR $\left(\mathrm{CDCl}_{3}, 200 \mathrm{MHz}\right.$, major signals) $\delta 7.83-7.41(\mathrm{~m}, 5 \mathrm{H}, \mathrm{Ar}), 3.73\left(\mathrm{t}, J=7.6 \mathrm{~Hz}, 2 \mathrm{H}, \mathrm{N}-\mathrm{CH}_{2}\right), 3.66(\mathrm{~s}$, $3 \mathrm{H}, \mathrm{OMe}), 2.29\left(\mathrm{t}, J=7.5 \mathrm{~Hz}, 2 \mathrm{H}, \mathrm{O}=\mathrm{C}-\mathrm{CH}_{2}\right), 1.71-1.47\left(\mathrm{~m}, 4 \mathrm{H}, 2 \mathrm{x} \mathrm{CH}_{2}\right), 1.34-1.13\left(\mathrm{~m}, 12 \mathrm{H},\left(\mathrm{CH}_{2}\right)_{6}\right)$.

${ }^{13} \mathbf{C}$ NMR $\left(\mathrm{CDCl}_{3}, 50 \mathrm{MHz}\right.$, major signals) $\delta 174.34,172.55,160.16(\mathrm{q}, J=39.3 \mathrm{~Hz}), 133.98,132.30,129.42$, $129.04,115.58(\mathrm{q}, J=286 \mathrm{~Hz}), 51.43,47.73,34.03,29.17,28.30,26.90,24.86$.

IR (neat, $\mathrm{cm}^{-1}$ ) 2926, 2853, 1736, 1719, 1704, 1388, 1165, 1088, 669.

TLC (3:1) hexane/EtOAc, permanganate, $\mathrm{R}_{f}=0.69$ (partial decomposition).

HRMS Decomposes to starting amide 27 (ESI).

\footnotetext{
${ }^{13}$ For formation of a similar compound: Nagubandi, S.; Fodor, G. Heterocycles 1981, 15, 165-177.
} 


\subsection{Synthesis of Amide Substrates for Mechanistic Studies and Supporting Table S3}

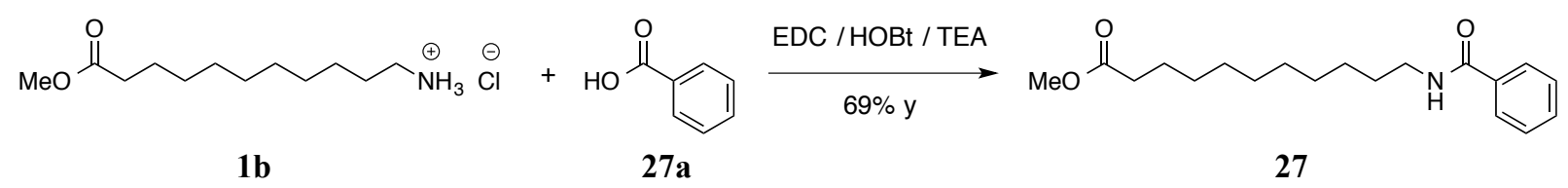

Synthesis of benzamide 27 Into a flask were added benzoic acid (27a, $660 \mathrm{mg}, 5.40 \mathrm{mmol}, 1$ equiv), amine hydrochloride $1 \mathbf{b}(1.50 \mathrm{~g}, 6.0 \mathrm{mmol}$, 1.1. equiv), EDC hydrochloride (1.60 g, $8.33 \mathrm{mmol}$, 1.5 equiv), hydroxybenzotriazole hydrate $(1.24 \mathrm{~g}, 8.33 \mathrm{mmol}, 1.5$ equiv), DCM $(18 \mathrm{~mL}, 0.3 \mathrm{M})$, and TEA $(2.00 \mathrm{~mL}, 14.0$ mmol, 2.6 equiv). The mixture was stirred $(20 \mathrm{~h})$, diluted with EtOAc $(60 \mathrm{~mL})$, washed with a 1:1 mixture $(120 \mathrm{~mL})$ of sodium carbonate ( $10 \%$ aqueous) and brine, then with a 1:1 mixture $(120 \mathrm{~mL})$ of sodium bisulfate (1 M aqueous) and brine, and then with a 1:1 mixture $(120 \mathrm{~mL})$ of water and brine, and dried with sodium sulfate. Volatiles were removed under reduced pressure to yield pure benzamide $27(1.22 \mathrm{~g}, 3.74 \mathrm{mmol}, 69 \%$ yield).

${ }^{1} \mathbf{H}$ NMR $\left(\mathrm{CDCl}_{3}, 200 \mathrm{MHz}\right) \delta 7.75(\mathrm{~d}, J=7.7 \mathrm{~Hz}, 2 \mathrm{H}, \mathrm{Ar}), 7.51-7.36$ (m, 3H, Ar), 6.09 (brs, $\left.1 \mathrm{H}, \mathrm{NH}\right), 3.66$ (s, $3 \mathrm{H}, \mathrm{OMe}), 3.45\left(\mathrm{q}, J=7.0 \mathrm{~Hz}, 2 \mathrm{H}, \mathrm{N}-\mathrm{CH}_{2}\right), 2.30\left(\mathrm{t}, J=7.5 \mathrm{~Hz}, 2 \mathrm{H}, \mathrm{O}=\mathrm{C}-\mathrm{CH}_{2}\right), 1.70-1.51\left(\mathrm{~m}, 4 \mathrm{H}, 2 \times \mathrm{CH}_{2}\right), 1.44-$ $1.19\left(\mathrm{~m}, 12 \mathrm{H},\left(\mathrm{CH}_{2}\right)_{6}\right)$.

${ }^{13} \mathrm{C}$ NMR $\left(\mathrm{CDCl}_{3}, 50 \mathrm{MHz}\right) \delta 174.06,167.39,134.56,130.86,128.06,126.77,51.14,39.87,33.78,29.35$, $29.05,28.94,28.81,26.73,24.64$.

IR (neat, $\left.\mathrm{cm}^{-1}\right) 3375,2918,2849.1723,1634,1522,1175,714$.

TLC (3:1) hexane/EtOAc, UV, $\mathrm{R}_{f}=0.39$.

HRMS calculated for $\left[\mathrm{C}_{19} \mathrm{H}_{29} \mathrm{NO}_{3} \mathrm{H}\right]^{+}$, requires $m / z=320.2226$, found $m / z=320.2226(\mathrm{ESI})$.
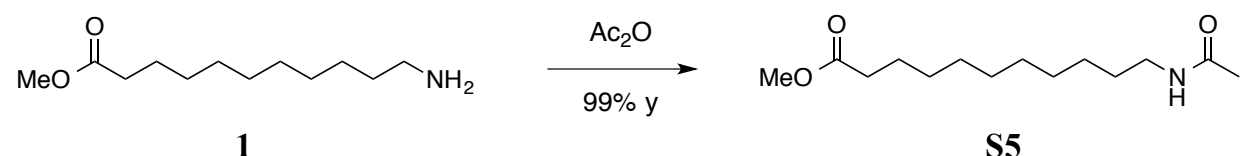

S5

Synthesis of acetamide S5 Into a flask were added amine 1 ( $1.47 \mathrm{~g}, 6.82 \mathrm{mmol}, 1$ equiv), EtOAc (10 mL, 0.7 $\mathrm{M})$, and acetic anhydride $(0.97 \mathrm{~mL}, 10.2 \mathrm{mmol}, 1.5$ equiv). The mixture was stirred $(22 \mathrm{~h})$. Volatiles were removed under reduced pressure to yield pure acetamide $\mathbf{S 5}(1.73 \mathrm{~g}, 6.75 \mathrm{mmol}, 99 \%$ yield, white solid).

${ }^{1} \mathbf{H}$ NMR $\left(\mathrm{CDCl}_{3}, 200 \mathrm{MHz}\right) \delta 5.45$ (brs, $\left.1 \mathrm{H}, \mathrm{NH}\right), 3.67$ (s, 3H, OMe), $3.23\left(\mathrm{q}, J=6.7 \mathrm{~Hz}, 2 \mathrm{H}, \mathrm{N}-\mathrm{CH}_{2}\right), 2.31$ (t, $\left.J=7.5 \mathrm{~Hz}, 2 \mathrm{H}, \mathrm{O}=\mathrm{C}-\mathrm{CH}_{2}\right), 1.98\left(\mathrm{~s}, 3 \mathrm{H}, \mathrm{O}=\mathrm{C}-\mathrm{CH}_{3}\right), 1.69-1.39\left(\mathrm{~m}, 4 \mathrm{H}, 2 \mathrm{x} \mathrm{CH}_{2}\right), 1.39-1.20\left(\mathrm{~m}, 12 \mathrm{H},\left(\mathrm{CH}_{2}\right)_{6}\right)$.

${ }^{13} \mathbf{C}$ NMR $\left(\mathrm{CDCl}_{3}, 50 \mathrm{MHz}\right) \delta 174.28,169.93,51.40,39.63,34.05,29.56,29.37,29.16,26.84,24.88,23.31$.

IR (neat, $\mathrm{cm}^{-1}$ ) 3286, 2914, 2848, 1730, 1636, 1556, 1177, 719, 608.

HRMS calculated for $\left[\mathrm{C}_{14} \mathrm{H}_{27} \mathrm{NO}_{3} \mathrm{H}\right]^{+}$, requires $\mathrm{m} / z=258.2069$, found $\mathrm{m} / z=258.2062$ (ESI).

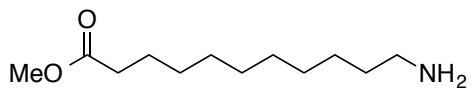

1

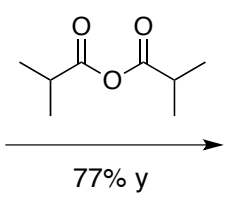

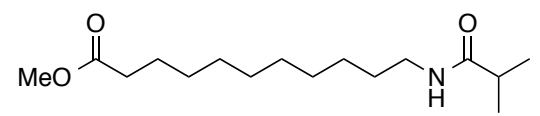

S6

Synthesis of amide S6 Into a flask were added amine 1 (1.00 g, $4.65 \mathrm{mmol}, 1$ equiv), EtOAc (5 mL, $1 \mathrm{M})$, and isobutyric anhydride $(1.20 \mathrm{~mL}, 6.97 \mathrm{mmol}, 1.5$ equiv, stench). The mixture was stirred $(6 \mathrm{~h})$, diluted with EtOAc $(30$ $\mathrm{mL})$, washed with sodium carbonate $(10 \%$ aqueous, $30 \mathrm{~mL})$, and dried with sodium sulfate. Volatiles were removed under reduced pressure, the crude product was purified by recrystallization ( $20 \mathrm{~mL}$ hot hexane), and the isolated solid was dried under vacuum to yield pure amide $\mathbf{S 6}$ (1.02 g, $3.57 \mathrm{mmol}, 77 \%$ yield, white solid).

${ }^{1} \mathbf{H}$ NMR $\left(\mathrm{CDCl}_{3}, 200 \mathrm{MHz}\right) \delta 5.42$ (brs, 1H, NH), 3.66 (s, 3H, OMe), 3.24 (q, J=6.5 Hz, 2H, N-CH $), 2.41-$ $2.45\left(\mathrm{~m}, 3 \mathrm{H}, \mathrm{Pr}, \mathrm{O}=\mathrm{C}-\mathrm{CH}_{2}\right), 1.68-1.40\left(\mathrm{~m}, 4 \mathrm{H}, 2 \times \mathrm{CH}_{2}\right), 1.36-1.20\left(\mathrm{~m}, 12 \mathrm{H},\left(\mathrm{CH}_{2}\right)_{6}\right), 1.15(\mathrm{~d}, J=7.5 \mathrm{~Hz}, 6 \mathrm{H}$, iPr). 
${ }^{13} \mathbf{C}$ NMR $\left(\mathrm{CDCl}_{3}, 50 \mathrm{MHz}\right) \delta 176.84,174.05,51.14,39.10,35.23,33.80,29.40,29.03,26.64,24.76,19.43$. IR (neat, $\mathrm{cm}^{-1}$ ) 3301, 2919, 2852, 1731, 1635, 1542, 1172.

HRMS calculated for $\left[\mathrm{C}_{16} \mathrm{H}_{31} \mathrm{NO}_{3} \mathrm{H}\right]^{+}$, requires $m / z=286.2382$, found $m / z=286.2378$ (ESI).

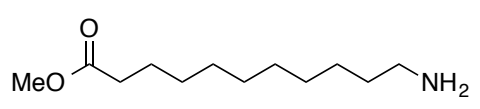

1

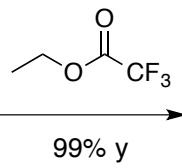

S7a

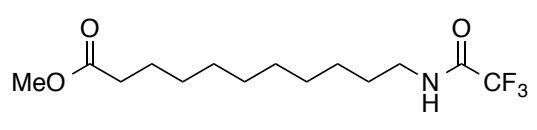

S7

Synthesis of trifluoroacetamide $\mathbf{S} 7$ Into a flask were added amine $\mathbf{1}(1.41 \mathrm{~g}, 6.55 \mathrm{mmol}, 1$ equiv), EtOAc (10 $\mathrm{mL}, 0.7 \mathrm{M})$, and ethyl trifluroacetate $(\mathbf{S} 7 \mathrm{a}, 1.17 \mathrm{~mL}, 9.83 \mathrm{mmol}, 1.5$ equiv). The mixture was stirred $(22 \mathrm{~h})$. Volatiles were removed under reduced pressure to yield pure trifluoroacetamide $\mathbf{S} 7$ ( $2.02 \mathrm{~g}, 6.50 \mathrm{mmol}, 99 \%$ yield). Compound analysis is consistent with published data. ${ }^{14}$

${ }^{1} \mathbf{H ~ N M R}\left(\mathrm{CDCl}_{3}, 200 \mathrm{MHz}\right) \delta 6.27(\mathrm{brs}, 1 \mathrm{H}, \mathrm{NH}), 3.67(\mathrm{~s}, 3 \mathrm{H}, \mathrm{OMe}), 3.36\left(\mathrm{q}, J=6.7 \mathrm{~Hz}, 2 \mathrm{H}, \mathrm{N}-\mathrm{CH}_{2}\right), 2.30(\mathrm{t}$, $\left.J=7.4 \mathrm{~Hz}, 2 \mathrm{H}, \mathrm{O}=\mathrm{C}-\mathrm{CH}_{2}\right), 1.73-1.47\left(\mathrm{~m}, 4 \mathrm{H}, 2 \mathrm{x} \mathrm{CH}_{2}\right), 1.43-1.13\left(\mathrm{~m}, 12 \mathrm{H},\left(\mathrm{CH}_{2}\right)_{6}\right)$.

TLC (3:1) hexane/EtOAc, permanganate, $\mathrm{R}_{f}=0.64$.

${ }^{14}$ Compound data from: Miyamoto, K.; Sei, Y.; Yamaguchi, K.; Ochiai, M. J. Am. Chem. Soc. 2009, 131, 13821383. 


\subsection{Synthesis of $N$-Nitrosoamides for Supporting Tables S1 and S2}<smiles>[R]C(=O)NCCCCCCCCCCC(=O)OC</smiles>

Standard nitrosylation procedure Into a vial were added the required amide $(150 \mathrm{mg})$ and the required solvent mixture (typically $0.3 \mathrm{M}$ in 5:1 acetic anhydride / acetic acid unless specified otherwise). The vial was capped with a rubber septum, sealed with tape, and stirred in an ice bath $\left(0{ }^{\circ} \mathrm{C}, 15 \mathrm{~min}\right)$. To the mixture was added solid sodium nitrite ( 2 equiv, creating a blue solution and slowly evolving brown gas). The vial was capped with a rubber septum, sealed with tape, vented with a syringe packed with anhydrous calcium sulfate, and stirred ( $2 \mathrm{~h})$. To the mixture was added additional sodium nitrite (2 equiv). The mixture was stirred (additional $4 \mathrm{~h}$ ), diluted with EtOAc $(5 \mathrm{~mL})$, washed with cold water $(5 \mathrm{~mL})$ and then cold sodium carbonate $(10 \%$ aqueous, $3 \times 5 \mathrm{~mL})$, diluted with cold sodium carbonate ( $10 \%$ aqueous, $5 \mathrm{~mL}$ ), and stirred rapidly (30 min, to quench any remaining anhydride). The organic phase was isolated, washed with potassium phosphate monobasic (1 M aqueous, $\mathrm{pH} 5,5 \mathrm{~mL}$ ), and dried with sodium sulfate. Volatiles were removed under reduced pressure to yield the crude product, which was analyzed by ${ }^{1} \mathrm{H}$ NMR to determine the conversion and product distribution. Crude $N$-nitrosoamides $\mathbf{S 1}-\mathbf{S 3}$ were pure enough to not require additional purification.

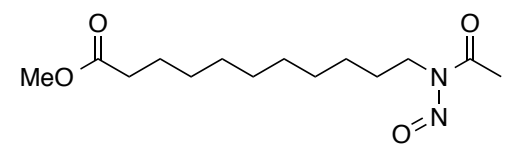

S1

${ }^{1} \mathbf{H}$ NMR $\left(\mathrm{CDCl}_{3}, 200 \mathrm{MHz}\right) \delta 3.74\left(\mathrm{t}, J=7.2 \mathrm{~Hz}, 2 \mathrm{H}, \mathrm{N}-\mathrm{CH}_{2}\right), 3.67$ (s, 3H, OMe), 2.76 (s, 3H, Ac), 2.30 (t, $J$ $\left.=7.5,2 \mathrm{H}, \mathrm{O}=\mathrm{C}-\mathrm{CH}_{2}\right), 1.70-1.52\left(\mathrm{~m}, 2 \mathrm{H}, \mathrm{CH}_{2}\right), 1.41-1.12\left(\mathrm{~m}, 14 \mathrm{H},\left(\mathrm{CH}_{2}\right)_{7}\right)$.

${ }^{13} \mathrm{C} \mathrm{NMR}\left(\mathrm{CDCl}_{3}, 50 \mathrm{MHz}\right) \delta 174.60,174.36,51.45,38.68,34.07,29.26,29.14,29.08,28.99,26.87,26.67$, 24.90, 22.52 .

IR $\left(\right.$ neat, $\left.\mathrm{cm}^{-1}\right)$ 2927, 2855, 1732, 1504, 1115, 952.

TLC (3:1) hexane/ $\mathrm{Et}_{2} \mathrm{O}$, UV \& permanganate, $\mathrm{R}_{f}=0.55$.

HRMS Decomposes to ester S1a (ESI).

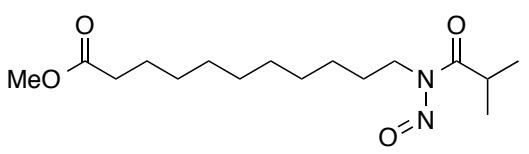

S2

${ }^{1} \mathbf{H}$ NMR $\left(\mathrm{CDCl}_{3}, 200 \mathrm{MHz}\right) \delta 3.88(\mathrm{sept}, J=6.8 \mathrm{~Hz}, 1 \mathrm{H}, \mathrm{iPr}), 3.74\left(\mathrm{t}, J=6.7 \mathrm{~Hz}, 2 \mathrm{H}, \mathrm{N}-\mathrm{CH}_{2}\right), 3.67(\mathrm{~s}, 3 \mathrm{H}$, $\mathrm{OMe}), 2.30\left(\mathrm{t}, J=7.5 \mathrm{~Hz}, 2 \mathrm{H}, \mathrm{O}=\mathrm{C}-\mathrm{CH}_{2}\right), 1.69-1.52\left(\mathrm{~m}, 2 \mathrm{H}, \mathrm{CH}_{2}\right), 1.31(\mathrm{~d}, J=6.9 \mathrm{~Hz}, 6 \mathrm{H}, \mathrm{Pr}), 1.27-1.11(\mathrm{~m}$, $\left.14 \mathrm{H},\left(\mathrm{CH}_{2}\right)_{6}\right)$.

${ }^{13} \mathbf{C}$ NMR $\left(\mathrm{CDCl}_{3}, 200 \mathrm{MHz}\right) \delta 181.02,174.35,51.44,38.73,34.06,32.62,29.26,29.11,28.99,26.80,26.60$, $24.89,19.15$.

IR (neat, $\left.\mathrm{cm}^{-1}\right)$ 2928, 2855, 1735, 1502, 1170, 1002.

TLC (3:1) hexane/Et $\mathrm{t}_{2} \mathrm{O}$, UV \& permanganate, $\mathrm{R}_{f}=0.75$.

HRMS Decomposes to ester S2a (ESI). 


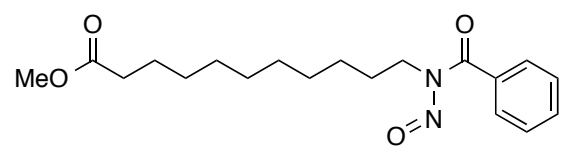

S3

To allow better solubility of the substrate, the reaction to make $N$-nitrosobenzamide $\mathbf{S 3}$ was run at $0.13 \mathrm{M}$.

${ }^{1} \mathbf{H}$ NMR $\left(\mathrm{CDCl}_{3}, 200 \mathrm{MHz}\right) \delta 7.75(\mathrm{~d}, J=7.8 \mathrm{~Hz}, 2 \mathrm{H}, \mathrm{Ar}), 7.64-7.42(\mathrm{~m}, 3 \mathrm{H}, \mathrm{Ar}), 3.93(\mathrm{t}, J=7.4 \mathrm{~Hz}, 2 \mathrm{H}, \mathrm{N}-$ $\left.\mathrm{CH}_{2}\right), 3.67(\mathrm{~s}, 3 \mathrm{H}, \mathrm{OMe}), 2.30\left(\mathrm{t}, J=7.6 \mathrm{~Hz}, \mathrm{O}=\mathrm{C}-\mathrm{CH}_{2}\right), 1.70-1.41\left(\mathrm{~m}, 4 \mathrm{H}, 2 \times \mathrm{CH}_{2}\right), 1.38-1.14\left(\mathrm{~m}, 12 \mathrm{H},\left(\mathrm{CH}_{2}\right)_{6}\right)$.

${ }^{13} \mathrm{C} \mathrm{NMR}\left(\mathrm{CDCl}_{3}, 50 \mathrm{MHz}\right) \delta 174.32,173.13,133.12,132.37,130.60,128.12,51.45,39.73,34.10,29.29$, $29.17,26.98,26.75,24.93$.

IR (neat, $\left.\mathrm{cm}^{-1}\right)$ 2926, 2855, 1737, 1702, 1504, 1352, 1168, 1001, 715.

TLC (3:1) hexane/Et ${ }_{2} \mathrm{O}$, UV \& permanganate, $\mathrm{R}_{f}=0.50$.

HRMS (minor signal) calculated for $\left[\mathrm{C}_{19} \mathrm{H}_{28} \mathrm{~N}_{2} \mathrm{O}_{4} \mathrm{H}\right]^{+}$, requires $\mathrm{m} / z=349.2127$, found $\mathrm{m} / z=349.2129$ (ESI). The majority of the material decomposes to ester $\mathbf{S 3 a}$.

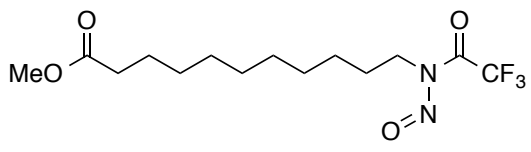

S4

$\mathrm{N}$-Nitrosotrifluoroacetamide $\mathbf{S} \mathbf{4}$ was not observed, as it rearranges spontaneously at ambient temperature. 


\subsection{Thermal Rearrangements of $N$-Nitrosoamides for Supporting Tables S1 and S2}

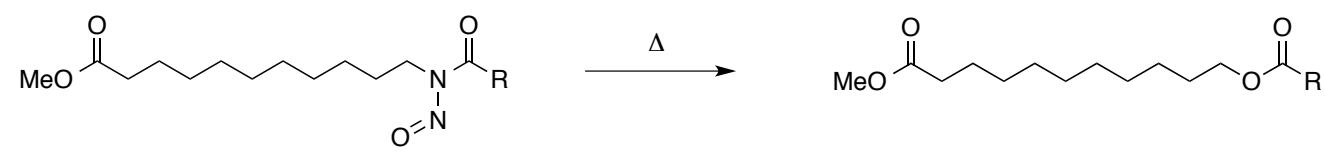

Standard rearrangement procedure Into a flask were added the crude $N$-nitrosoamide and the solvent $(0.1 \mathrm{M}$, toluene unless specified otherwise). Using an oil bath, the mixture was heated to the indicated temperature for the indicated time (typically 5 or 24 hours). Volatiles were removed under reduced pressure, and the crude mixture was analyzed by ${ }^{1} \mathrm{H}$ NMR to assess conversion and product distribution.

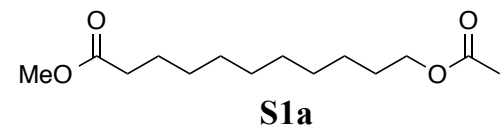

${ }^{1} \mathbf{H}$ NMR $\left(\mathrm{CDCl}_{3}, 200 \mathrm{MHz}\right) \delta 4.05\left(\mathrm{t}, J=6.7 \mathrm{~Hz}, 2 \mathrm{H}, \mathrm{O}-\mathrm{CH}_{2}\right), 3.67(\mathrm{~s}, 3 \mathrm{H}, \mathrm{OMe}), 2.30(\mathrm{t}, J=7.5 \mathrm{~Hz}, 2 \mathrm{H}$, $\left.\mathrm{O}=\mathrm{C}-\mathrm{CH}_{2}\right), 2.05\left(\mathrm{~s}, 3 \mathrm{H}, \mathrm{O}=\mathrm{C}-\mathrm{CH}_{3}\right), 1.70-1.50\left(\mathrm{~m}, 4 \mathrm{H}, 2 \mathrm{x} \mathrm{CH}_{2}\right), 1.35-1.21\left(\mathrm{~m}, 12 \mathrm{H},\left(\mathrm{CH}_{2}\right)_{6}\right)$.

${ }^{13} \mathrm{C}$ NMR $\left(\mathrm{CDCl}_{3}, 50 \mathrm{MHz}\right) \delta 173.65,170.55,64.11,50.91,33.62,28.85,28.26,25.54,24.55,20.50$.

IR (neat, $\mathrm{cm}^{-1}$ ) 2926, 2855, 1736, 1234 .

TLC (3:1) hexane/EtOAc, permanganate, $\mathrm{R}_{f}=0.68$.

HRMS calculated for $\left[\mathrm{C}_{14} \mathrm{H}_{26} \mathrm{O}_{4} \mathrm{H}\right]^{+}$, requires $m / z=259.1909$, found $m / z=259.1904$ (ESI).

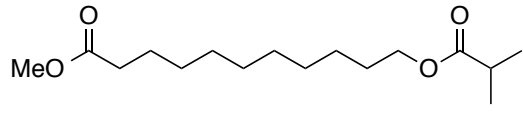

S2a

${ }^{1} \mathbf{H}$ NMR $\left(\mathrm{CDCl}_{3}, 200 \mathrm{MHz}\right) \delta 4.05\left(\mathrm{t}, J=6.7 \mathrm{~Hz}, 2 \mathrm{H}, \mathrm{O}-\mathrm{CH}_{2}\right), 3.67$ (s, 3H, OMe), 2.54 (sept, $J=7.0 \mathrm{~Hz}, 1 \mathrm{H}$, iPr), 2.31 (t, $\left.J=7.5 \mathrm{~Hz}, 2 \mathrm{H}, \mathrm{O}=\mathrm{C}-\mathrm{CH}_{2}\right), 1.70-1.51\left(\mathrm{~m}, 4 \mathrm{H}, 2 \times \mathrm{CH}_{2}\right), 1.36-1.24\left(\mathrm{~m}, 12 \mathrm{H},\left(\mathrm{CH}_{2}\right)_{6}\right), 1.16(\mathrm{~d}, J=7.0$ $\mathrm{Hz}, 6 \mathrm{H}, \mathrm{iPr})$.

${ }^{13} \mathbf{C}$ NMR $\left(\mathrm{CDCl}_{3}, 50 \mathrm{MHz}\right) \delta 176.81,173.87,64.05,51.10,33.77,28.97,28.40,25.65,24.69,18.76$.

IR (neat, $\left.\mathrm{cm}^{-1}\right) 2928,2855,1735,1193,1157$.

TLC (3:1) hexane/EtOAc, permanganate, $\mathrm{R}_{f}=0.69$.

HRMS calculated for $\left[\mathrm{C}_{16} \mathrm{H}_{30} \mathrm{O}_{4} \mathrm{H}\right]^{+}$, requires $m / z=287.222$, found $m / z=287.2214$ (ESI).

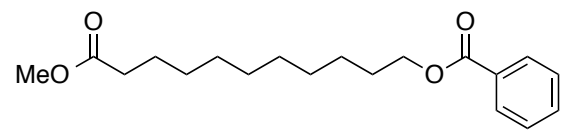

S3a

${ }^{1} \mathbf{H}$ NMR $\left(\mathrm{CDCl}_{3}, 200 \mathrm{MHz}\right) \delta 8.05(\mathrm{~d}, J=7.5 \mathrm{~Hz}, 2 \mathrm{H}, \mathrm{Ar}), 7.62-7.37(\mathrm{~m}, 3 \mathrm{H}, \mathrm{Ar}), 4.32(\mathrm{t}, J=6.6 \mathrm{~Hz}, 2 \mathrm{H}, \mathrm{O}-$ $\left.\mathrm{CH}_{2}\right) 3.67(\mathrm{~s}, 3 \mathrm{H}, \mathrm{OMe}), 2.30\left(\mathrm{t}, J=7.5 \mathrm{~Hz}, 2 \mathrm{H}, \mathrm{O}=\mathrm{C}-\mathrm{CH}_{2}\right), 1.84-1.51\left(\mathrm{~m}, 4 \mathrm{H}, 2 \times \mathrm{CH}_{2}\right), 1.51-1.17(\mathrm{~m}, 12 \mathrm{H}$, $\left.\left(\mathrm{CH}_{2}\right)_{6}\right)$.

${ }^{13} \mathrm{C}$ NMR $\left(\mathrm{CDCl}_{3}, 50 \mathrm{MHz}\right) \delta 174.32,166.67,132.77,130.48,129.51,128.29,65.10,51.44,34.08,29.22$, 28.68, 26.01, 24.93.

IR $\left(\right.$ neat, $\left.\mathrm{cm}^{-1}\right)$ 2927, 2855, 1738, 1717, 1271, 1109, 710 .

TLC (15:1) hexane/EtOAc, UV \& permanganate, $\mathrm{R}_{f}=0.30$.

HRMS calculated for $\left[\mathrm{C}_{19} \mathrm{H}_{28} \mathrm{O}_{4} \mathrm{H}\right]^{+}$, requires $\mathrm{m} / z=321.2066$, found $\mathrm{m} / \mathrm{z}=321.2060$ (ESI). 


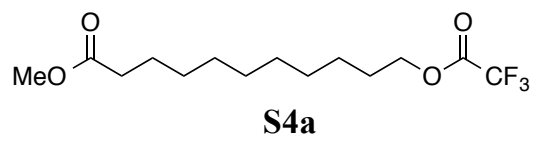

${ }^{1} \mathbf{H}$ NMR $\left(\mathrm{CDCl}_{3}, 200 \mathrm{MHz}\right) \delta 4.35\left(\mathrm{t}, J=6.7 \mathrm{~Hz}, 2 \mathrm{H}, \mathrm{O}-\mathrm{CH}_{2}\right), 3.67(\mathrm{~s}, 3 \mathrm{H}, \mathrm{OMe}), 2.31(\mathrm{t}, J=7.5 \mathrm{~Hz}, 2 \mathrm{H}$, $\left.\mathrm{O}=\mathrm{C}-\mathrm{CH}_{2}\right), 1.82-1.50\left(\mathrm{~m}, 4 \mathrm{H}, 2 \times \mathrm{CH}_{2}\right), 1.50-1.22\left(\mathrm{~m}, 12 \mathrm{H},\left(\mathrm{CH}_{2}\right)_{6}\right)$.

${ }^{13} \mathbf{C}$ NMR $\left(\mathrm{CDCl}_{3}, 50 \mathrm{MHz}\right) \delta 174.01,157.35(\mathrm{q}, J=41.9 \mathrm{~Hz}), 114.46(\mathrm{q}, J=286 \mathrm{~Hz}), 68.09,51.12,33.85$, $29.11,27.94,25.33,24.77$.

IR (neat, $\left.\mathrm{cm}^{-1}\right)$ 2929, 2857, 1784, 1738, 1217, 1144.

TLC (5:1) hexane/EtOAc, permanganate, $\mathrm{R}_{f}=0.78$ (partial decomposition on TLC plate).

HRMS calculated for $\left[\mathrm{C}_{14} \mathrm{H}_{23} \mathrm{~F}_{3} \mathrm{O}_{4} \mathrm{Na}\right]^{+}$, requires $m / z=335.1446$, found $m / z=335.1445$ (ESI). 


\section{NMR Spectra}

${ }^{1}$ H NMR Spectrum of 1

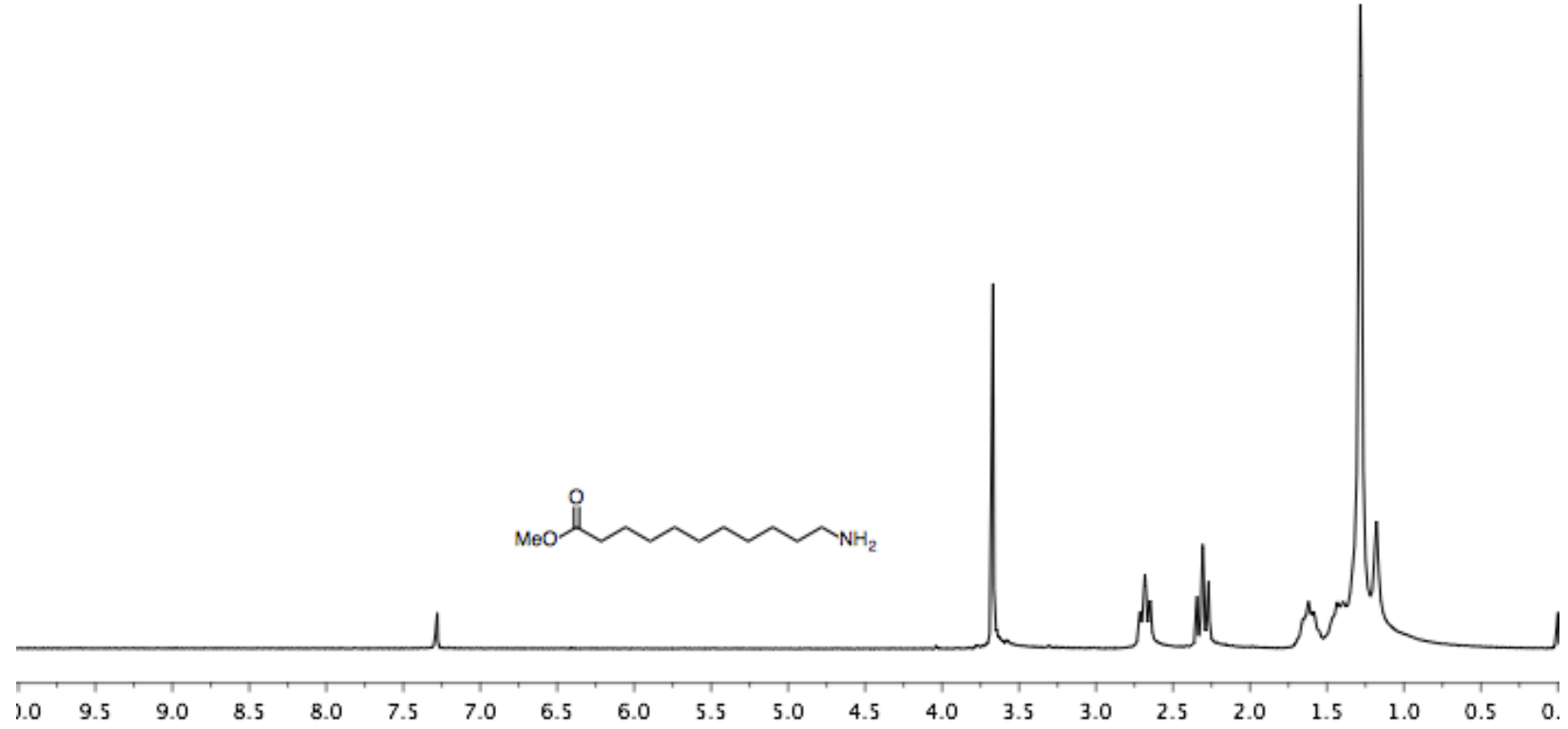

${ }^{1} \mathrm{H}$ NMR Spectrum of 2

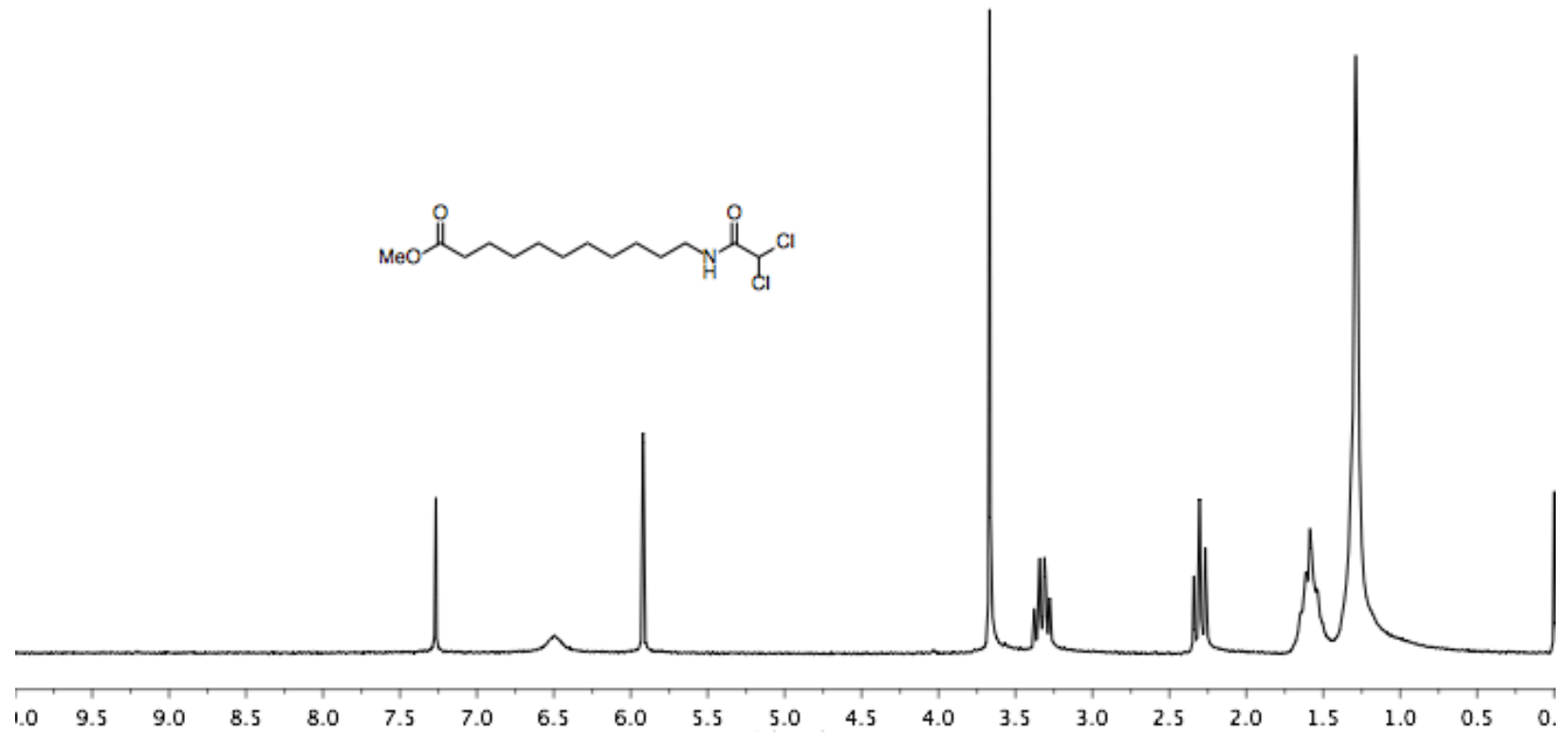


${ }^{13} \mathrm{C}$ NMR Spectrum of 2

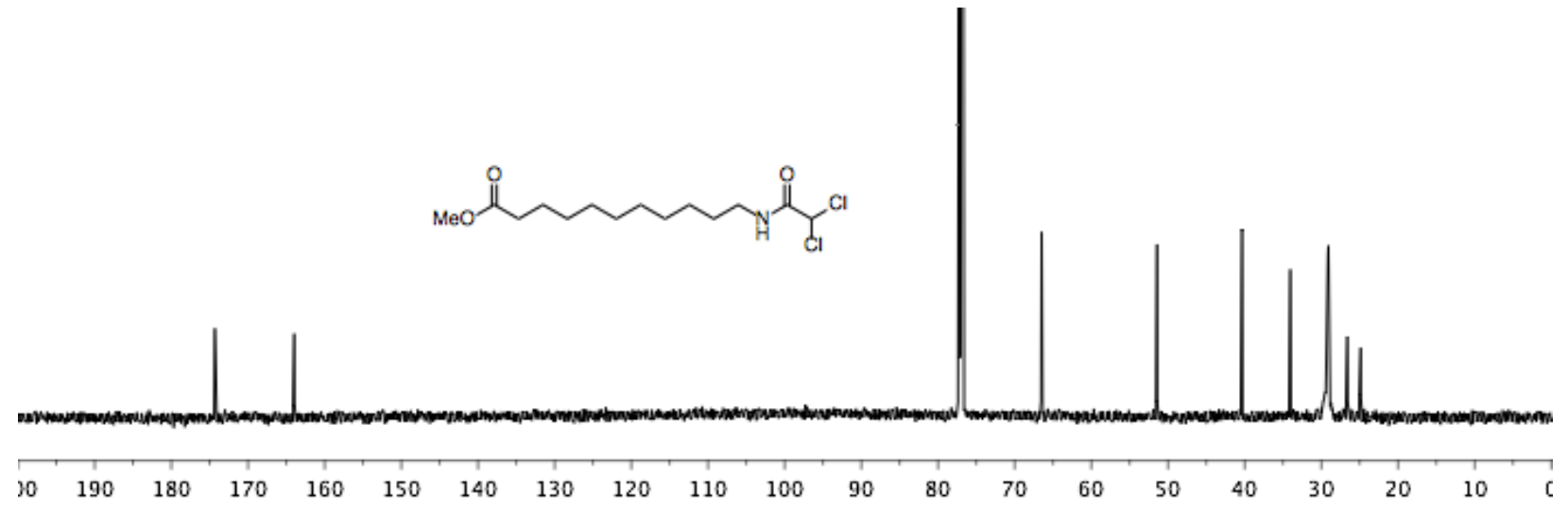

${ }^{1}$ H NMR Spectrum of 3

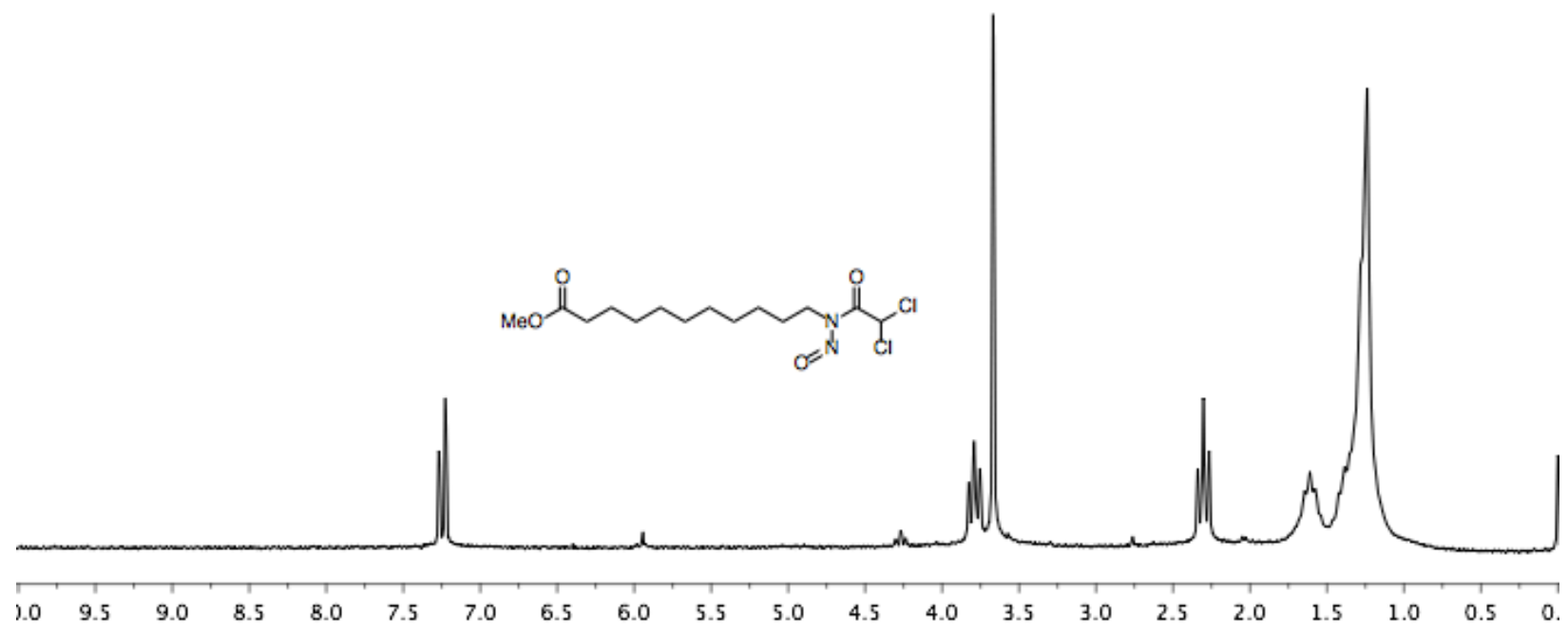


${ }^{13}$ C NMR Spectrum of 3

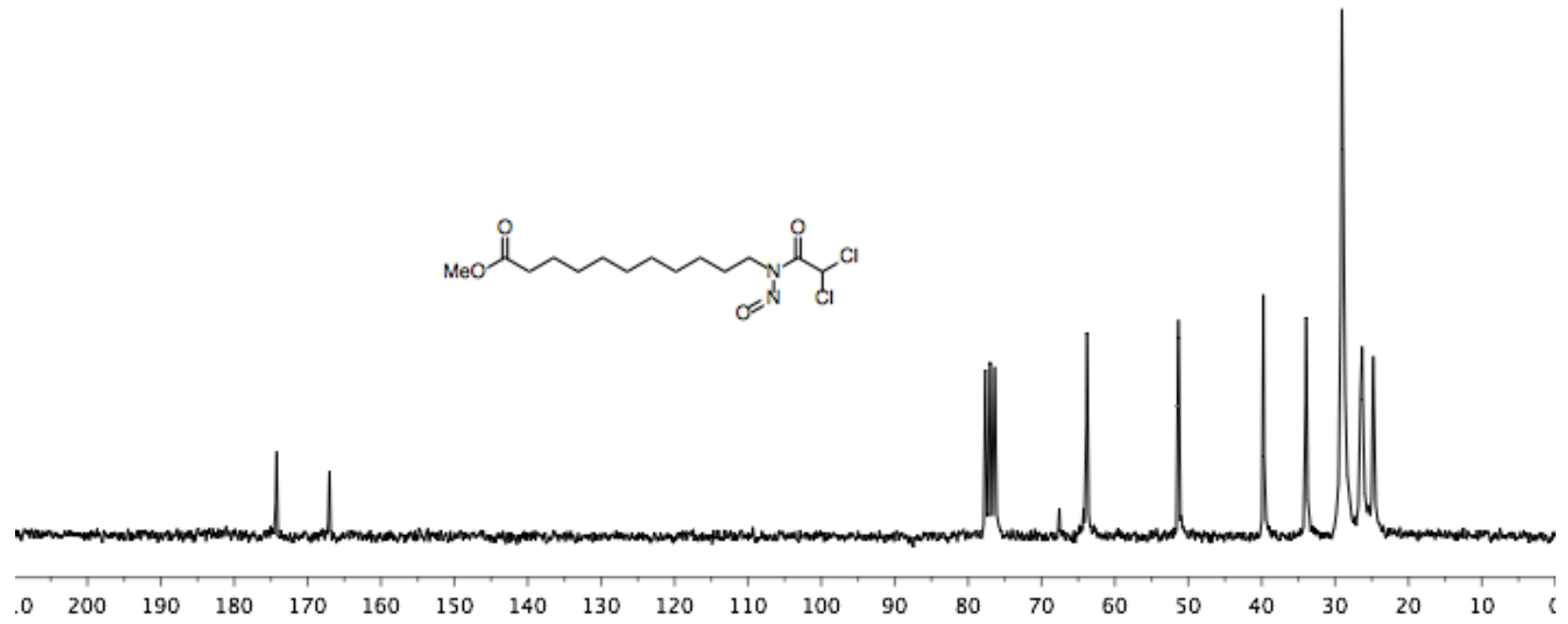

${ }^{1}$ H NMR Spectrum of 4

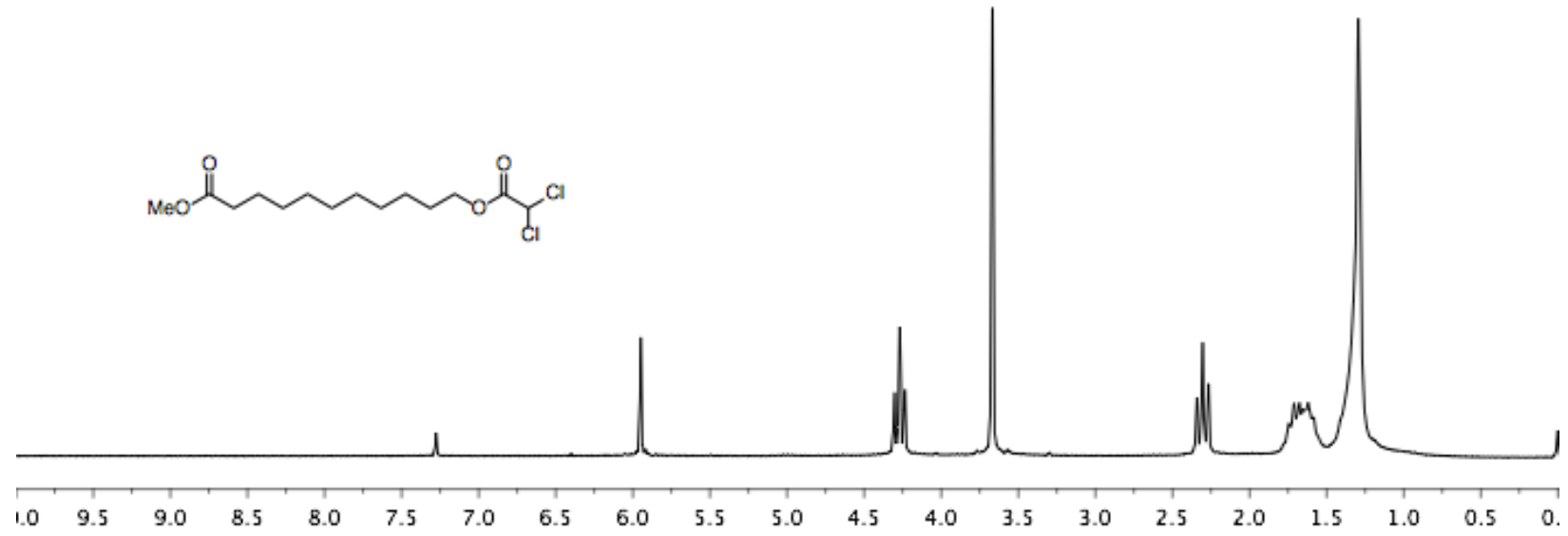

${ }^{13}$ C NMR Spectrum of 4

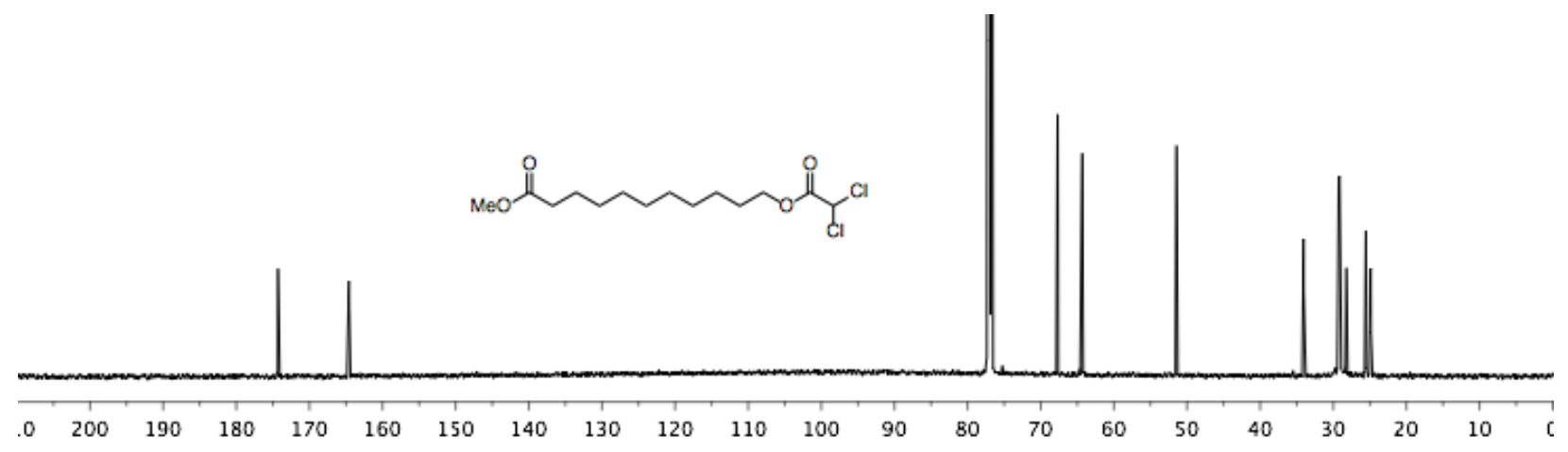


${ }^{1}$ H NMR Spectrum of 4a (contaminated with some $4 b$ )

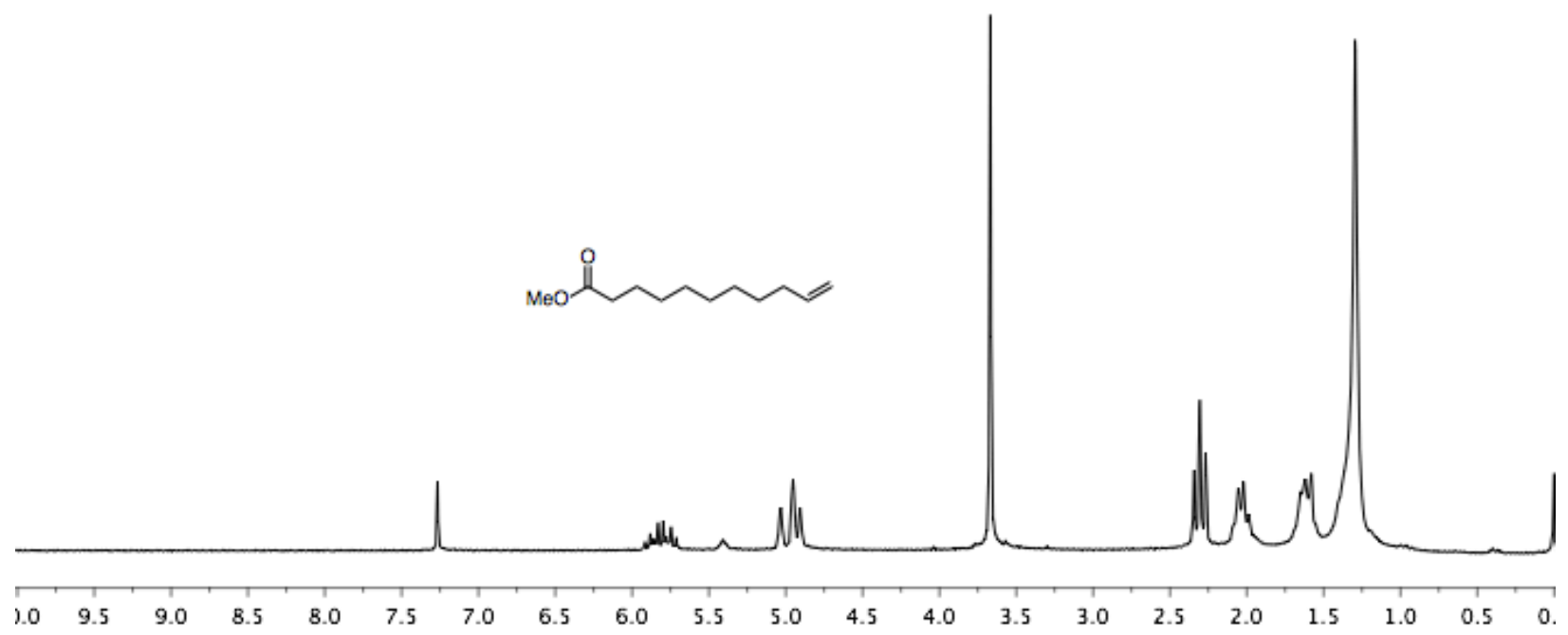

${ }^{1}$ H NMR Spectrum of 5

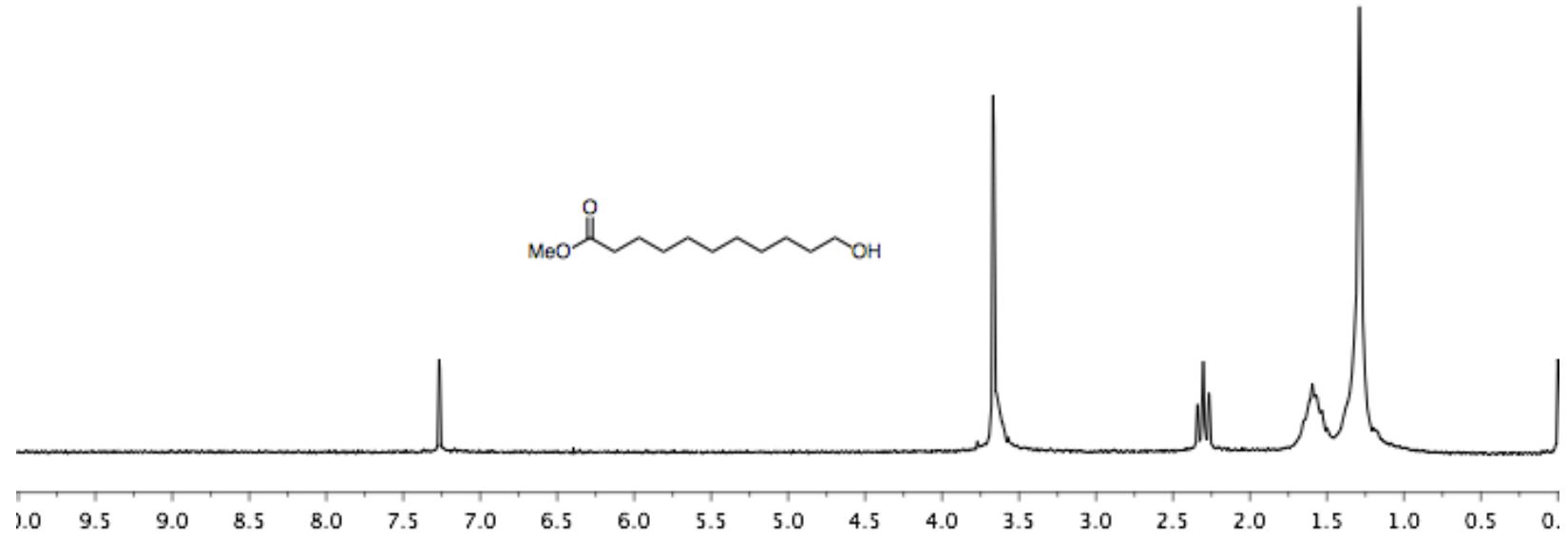


${ }^{1}$ H NMR Spectrum of 7

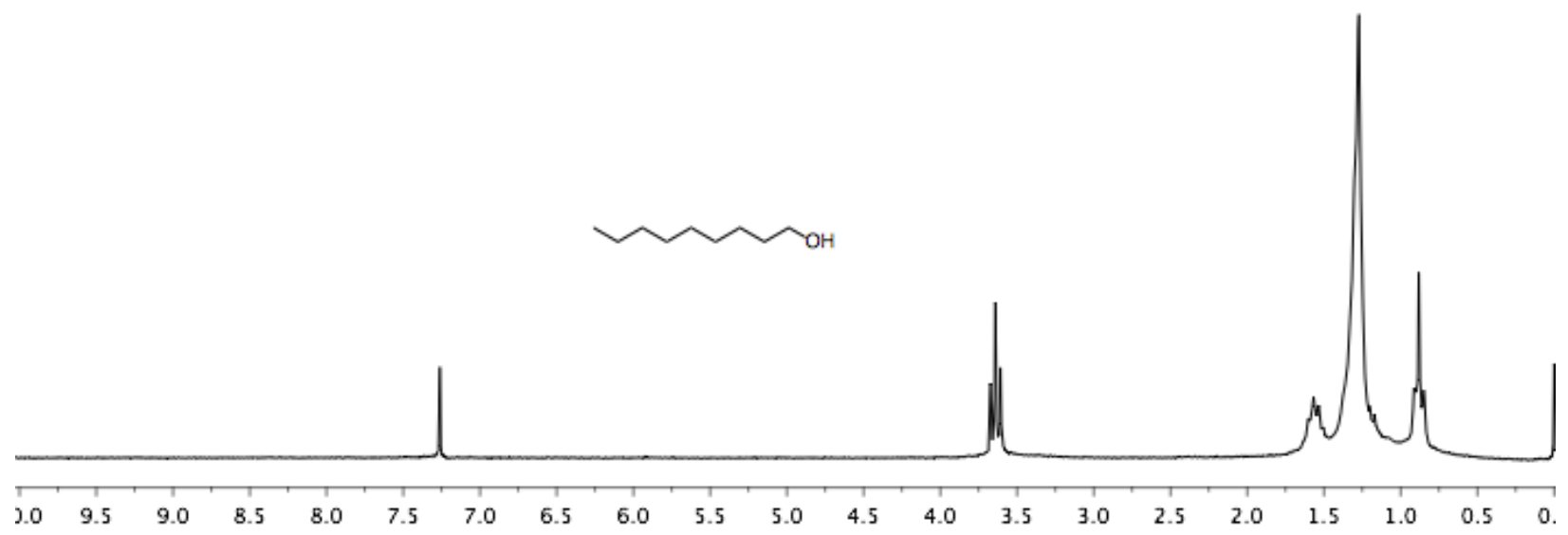

${ }^{1} \mathrm{H}$ NMR Spectrum of 9

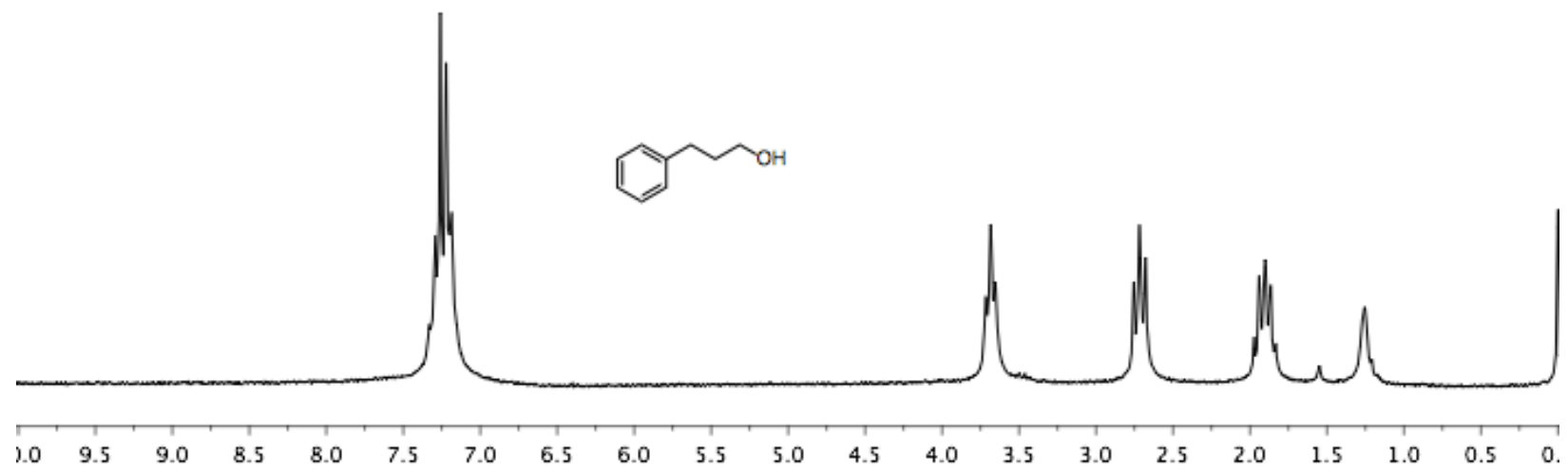

${ }^{1} \mathrm{H}$ NMR Spectrum of $10 \mathrm{~b}$

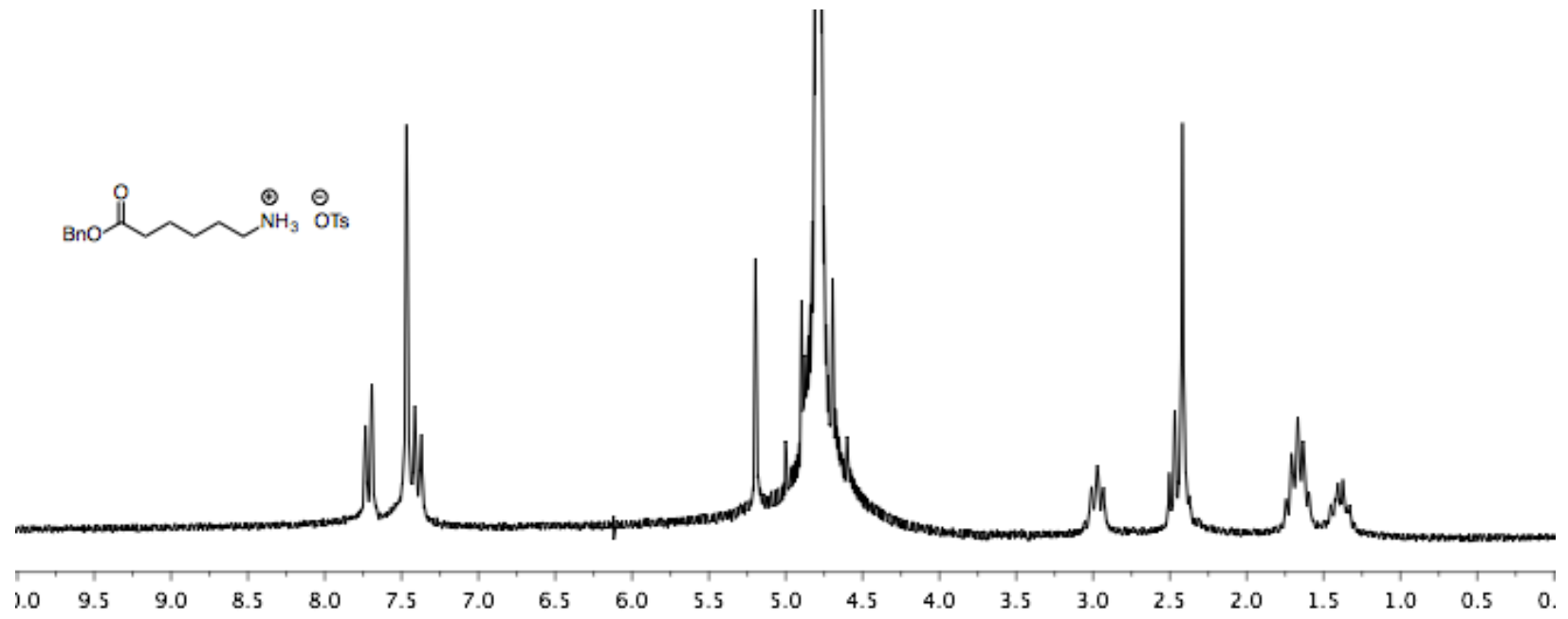


${ }^{1}$ H NMR Spectrum of 10

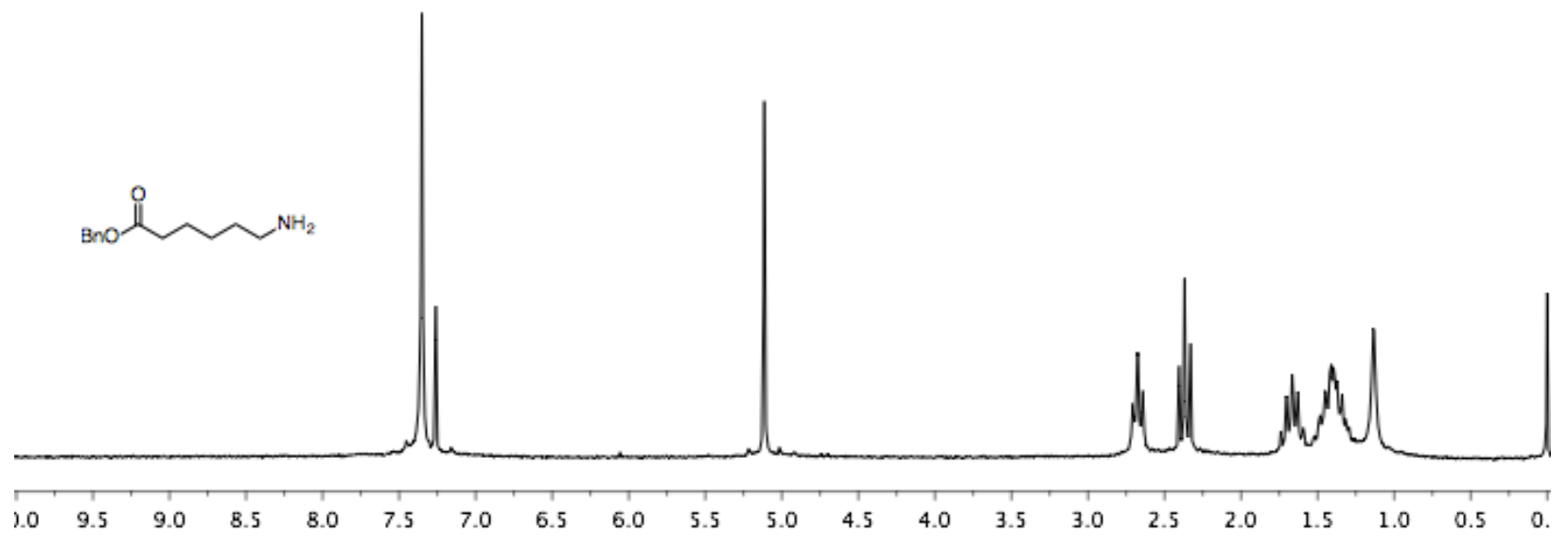

${ }^{1}$ H NMR Spectrum of 11

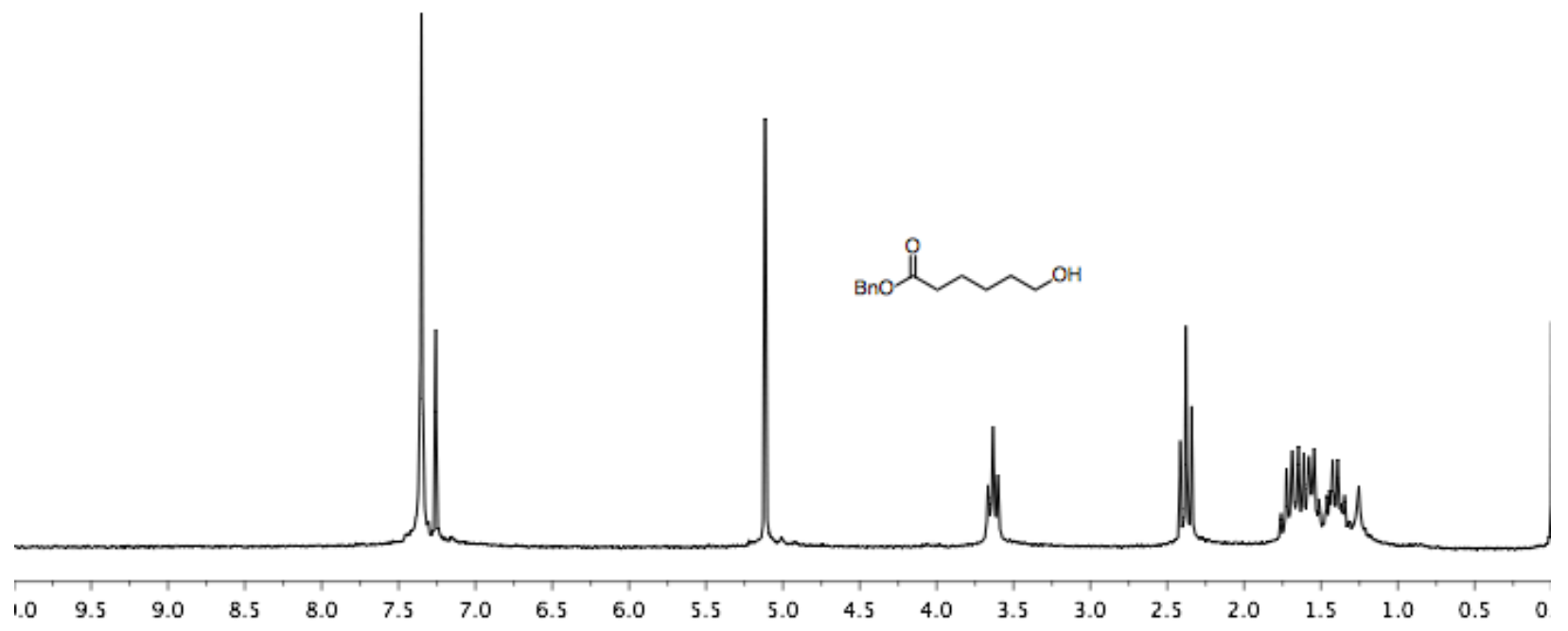


${ }^{1} \mathrm{H}$ NMR Spectrum of $12 \mathrm{~b}$

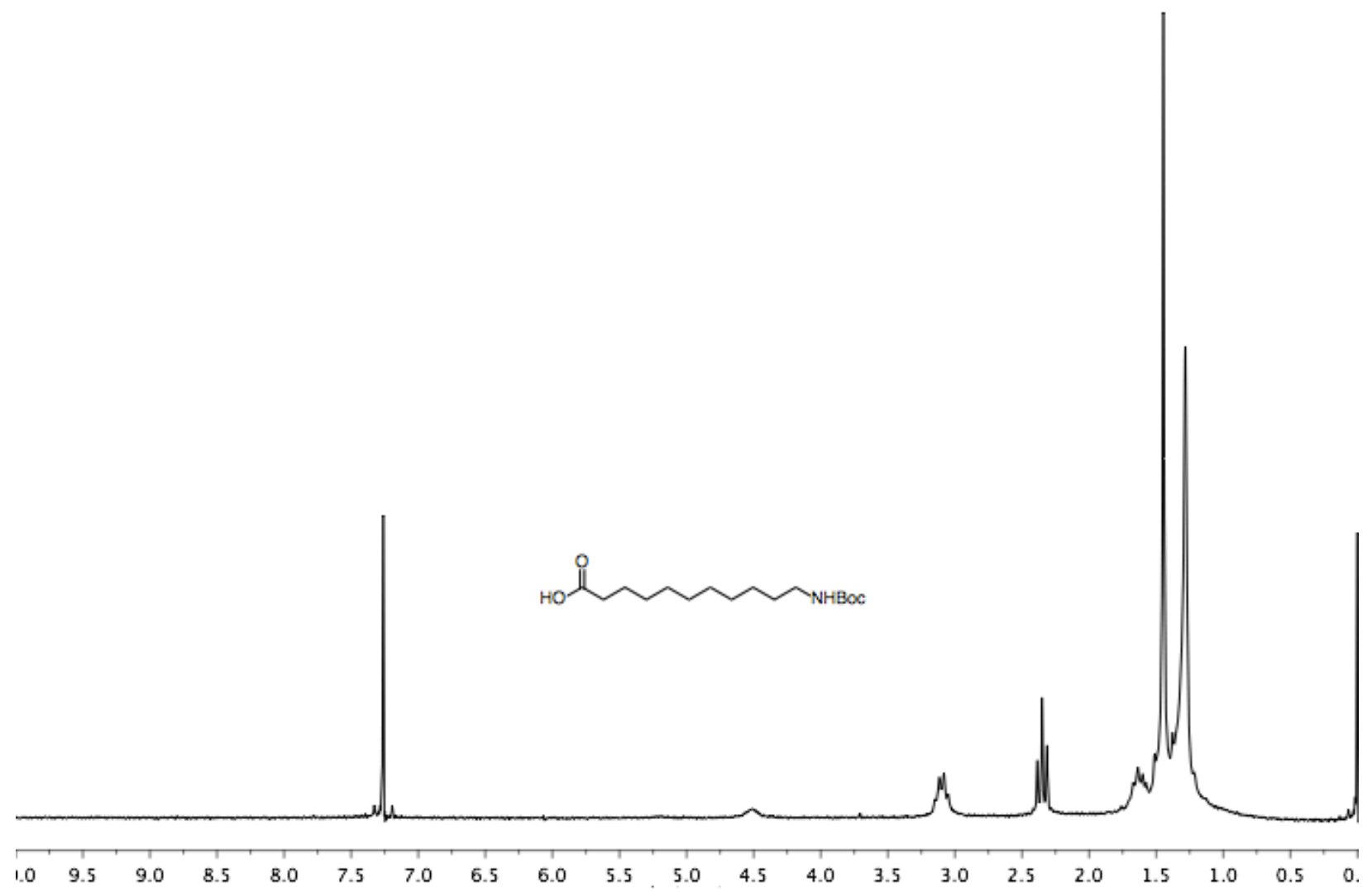


${ }^{1}$ H NMR Spectrum of 12d

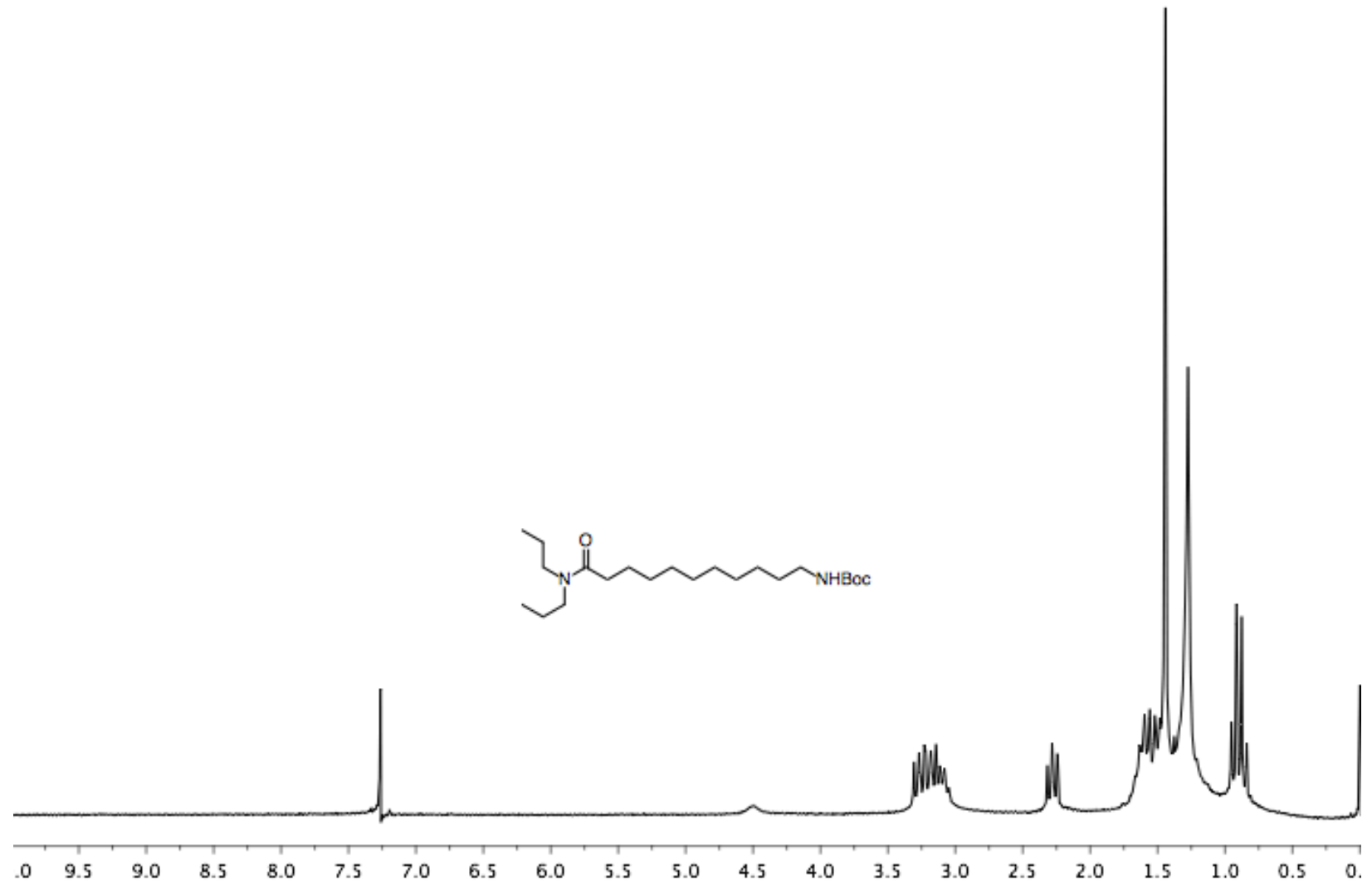

${ }^{13}$ C NMR Spectrum of $12 d$

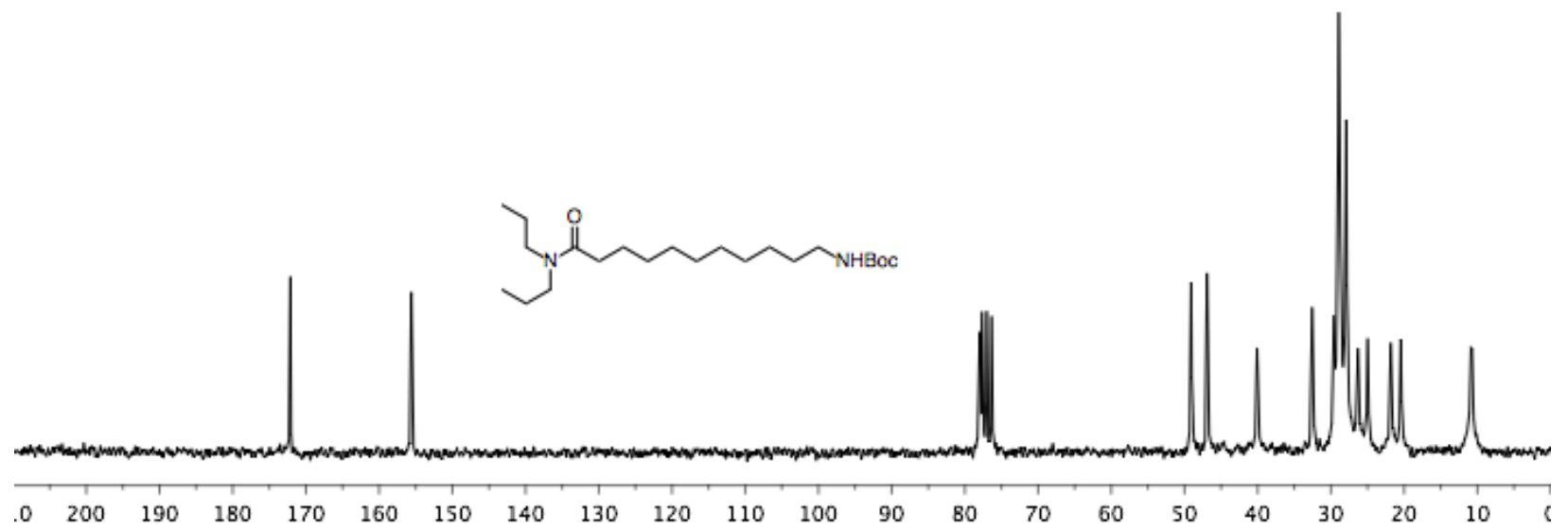


${ }^{1}$ H NMR Spectrum of 12

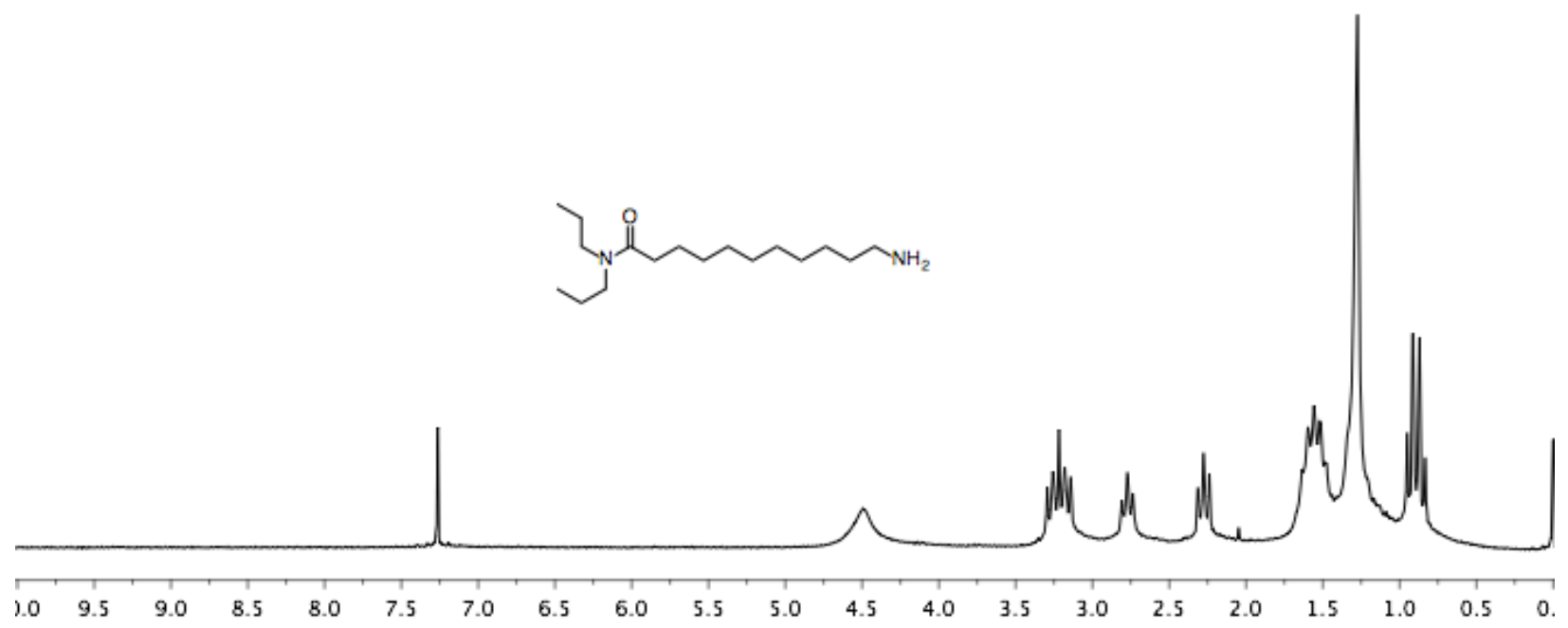

${ }^{13} \mathrm{C}$ NMR Spectrum of 12

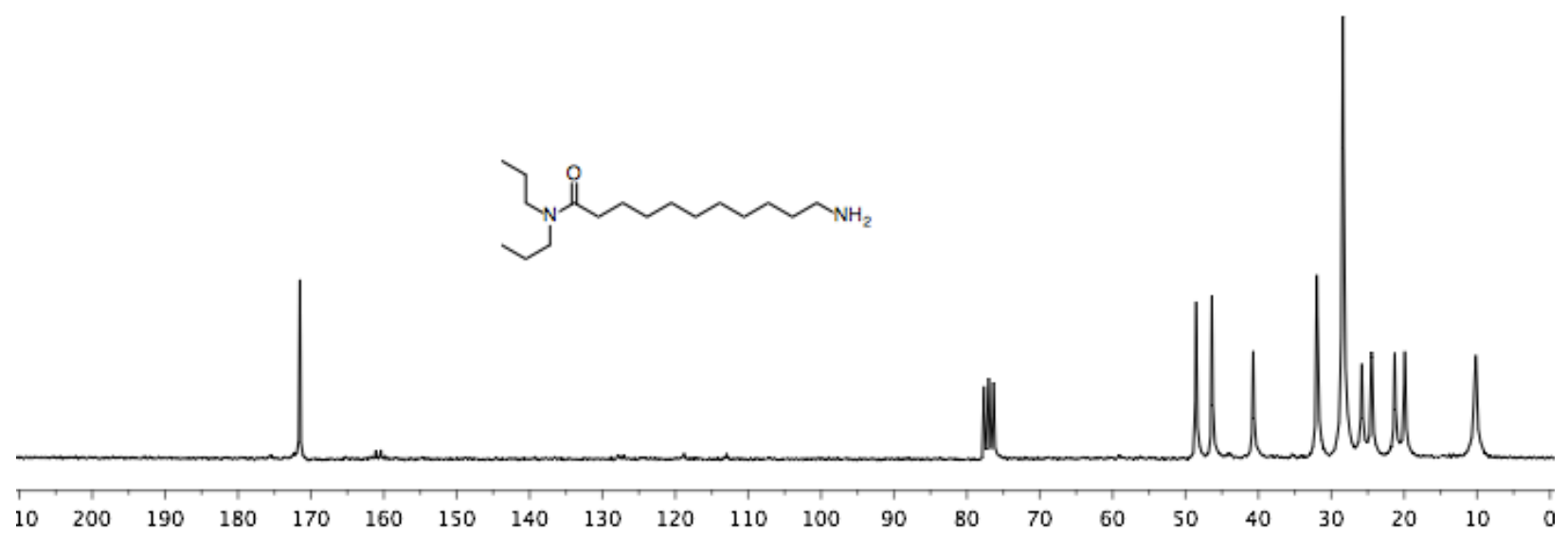


${ }^{1}$ H NMR Spectrum of 13

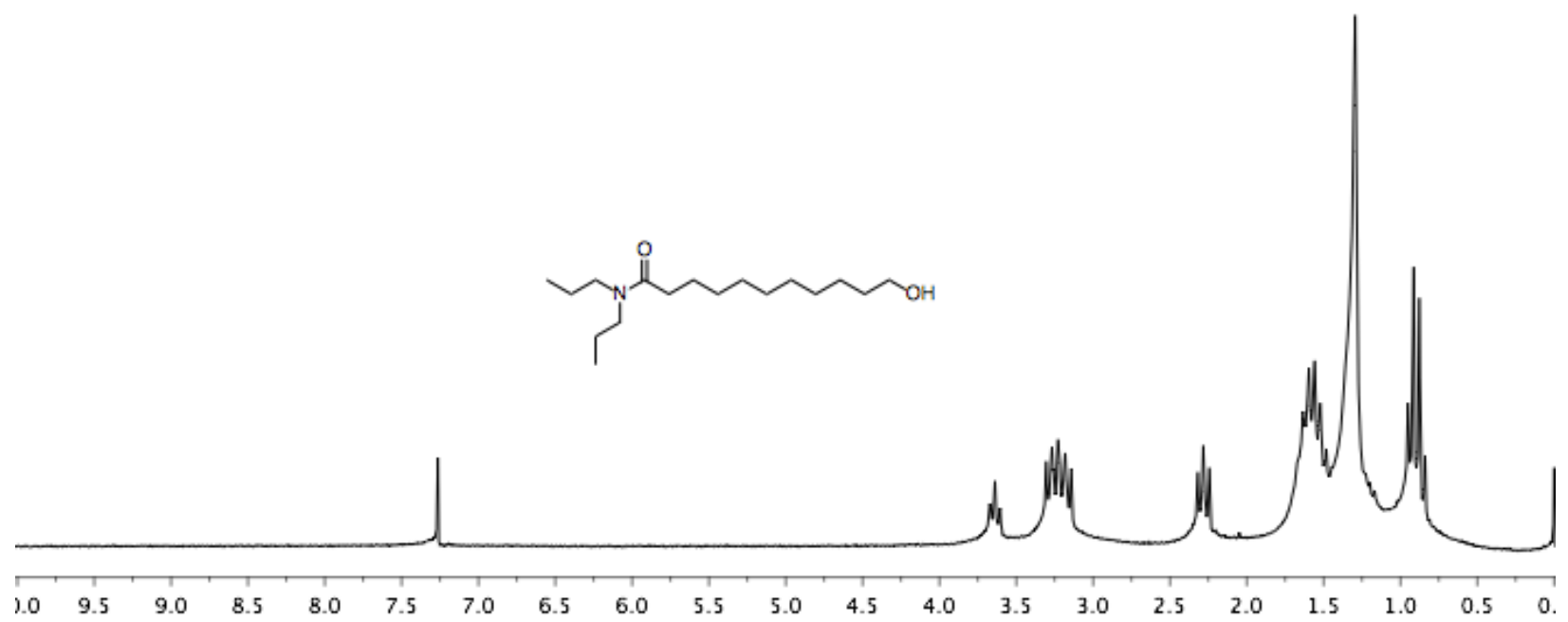

${ }^{13} \mathrm{C}$ NMR Spectrum of 13

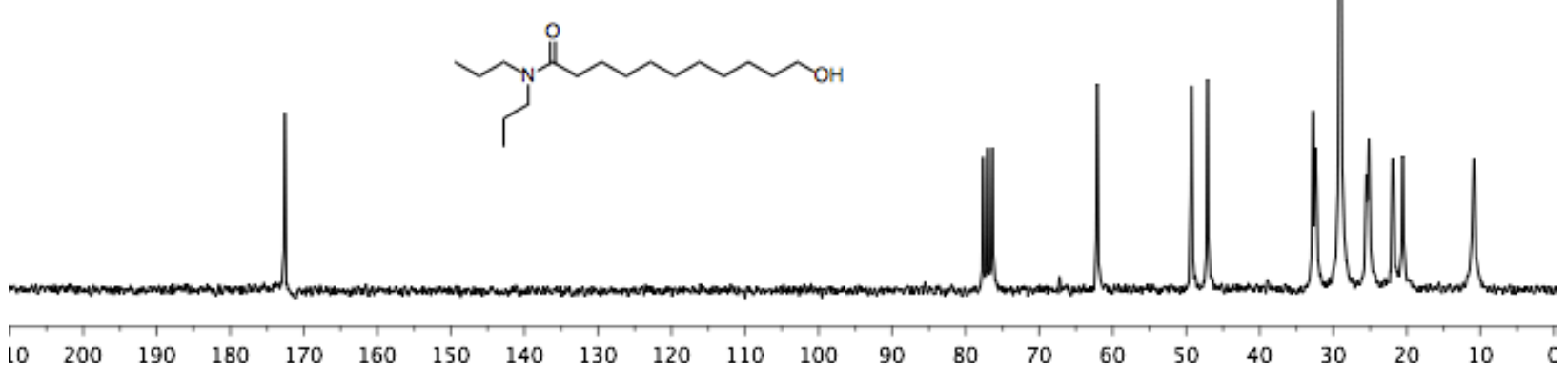


${ }^{1}$ H NMR Spectrum of $14 \mathrm{c}$

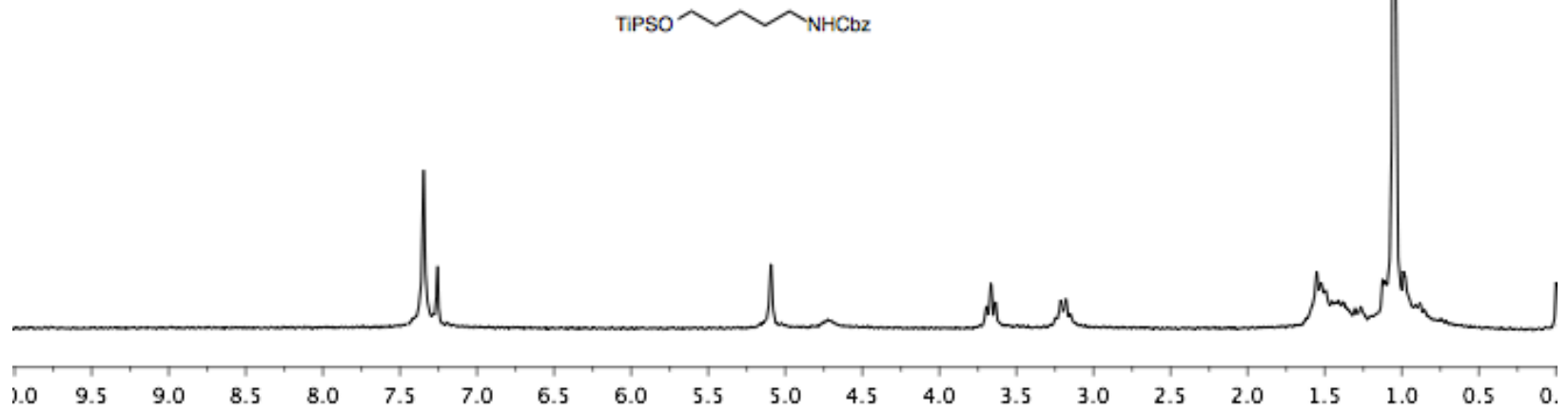

${ }^{13}$ C NMR Spectrum of $14 c$

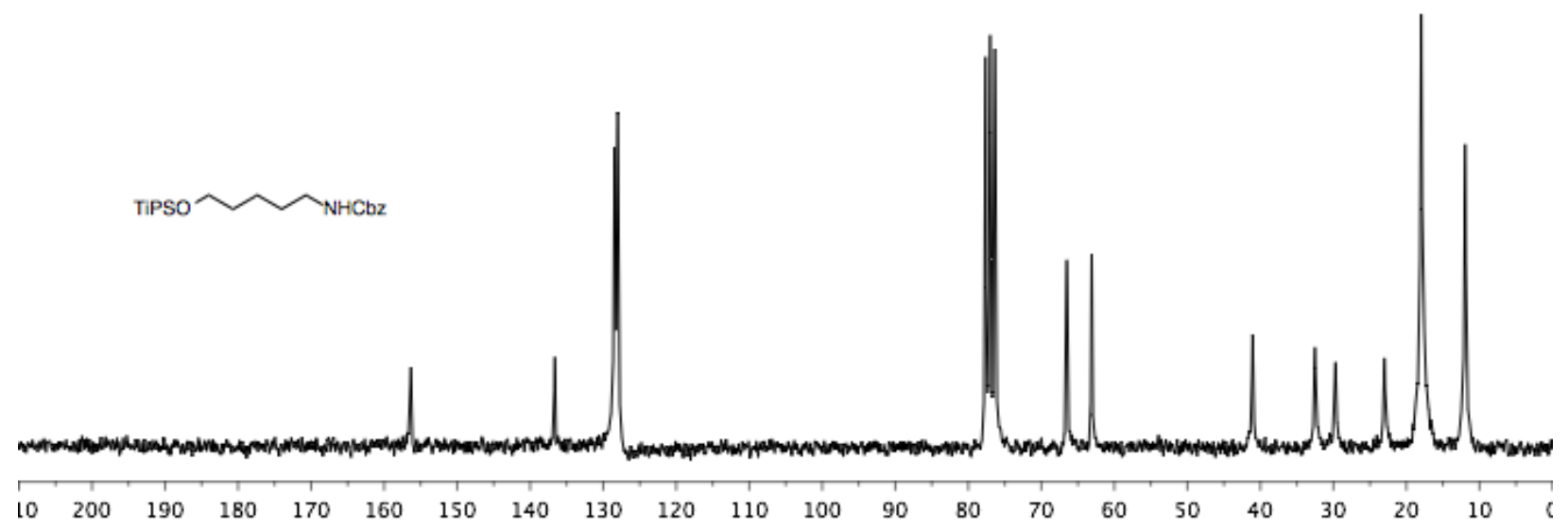


${ }^{1}$ H NMR Spectrum of 14

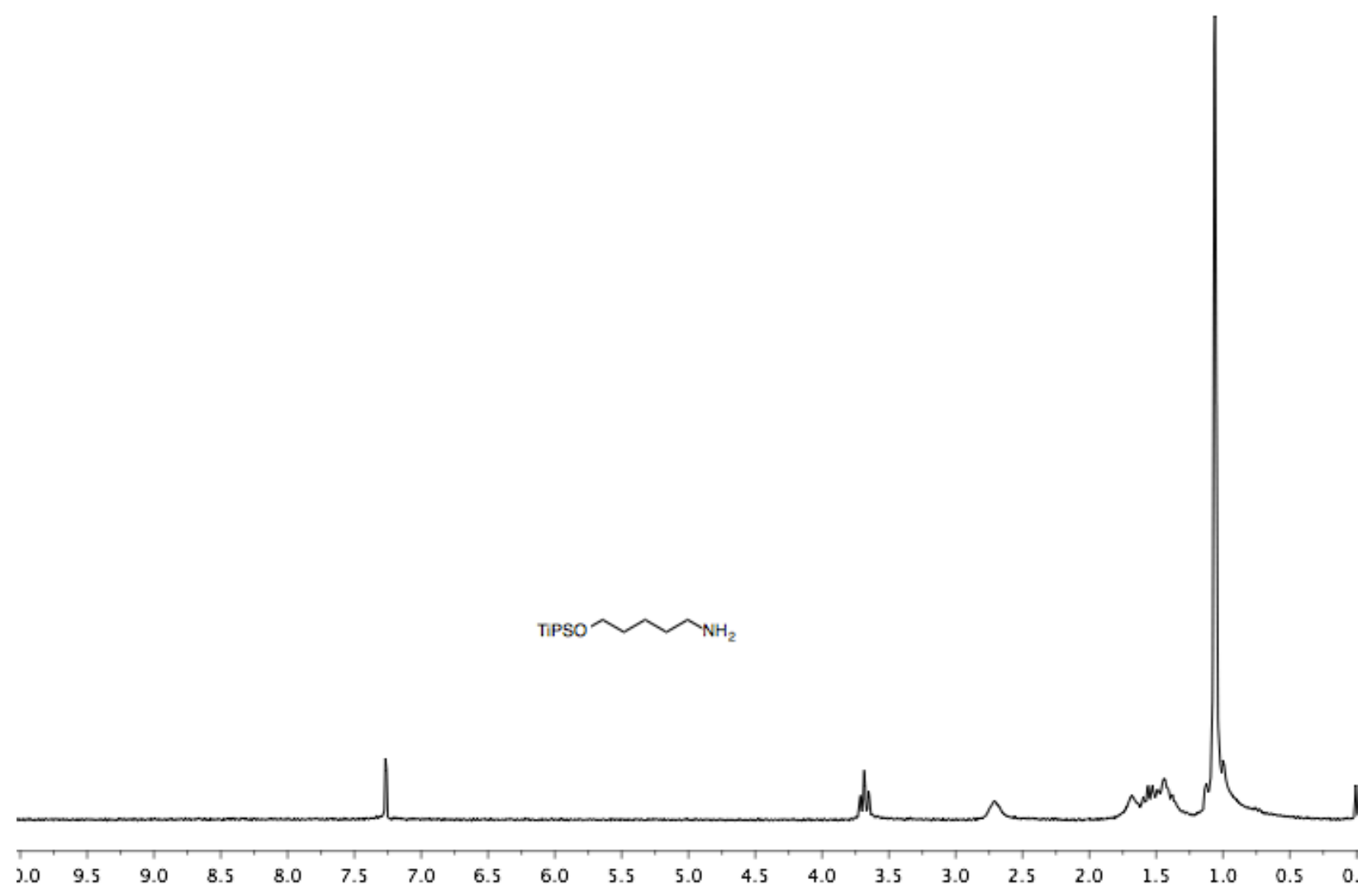

${ }^{13} \mathrm{C}$ NMR Spectrum of 14

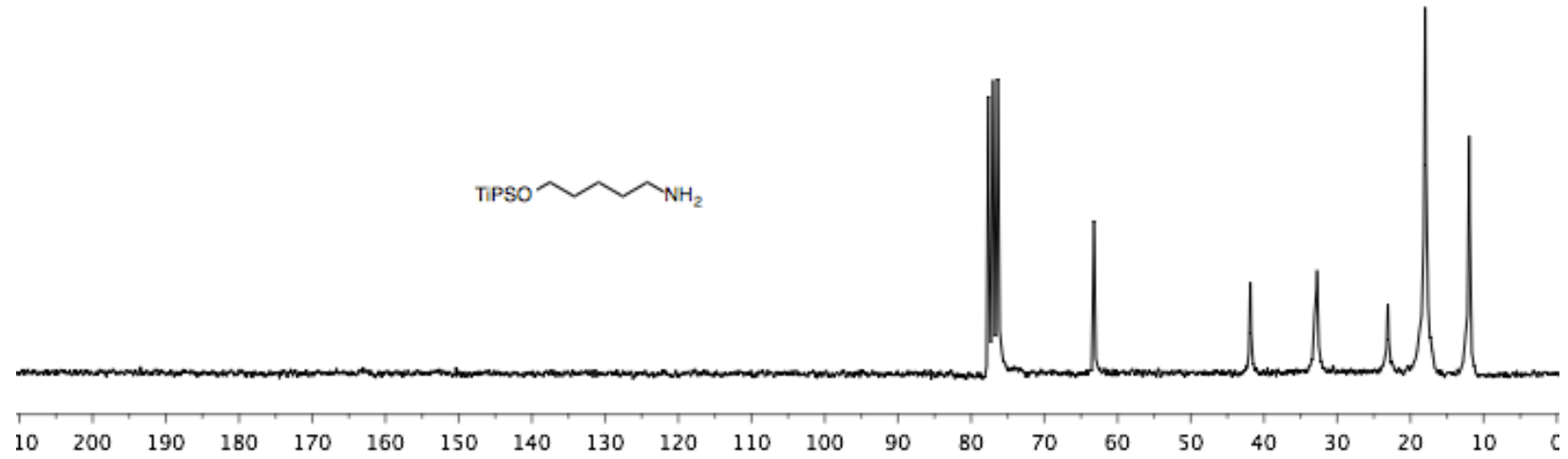


${ }^{1}$ H NMR Spectrum of 15

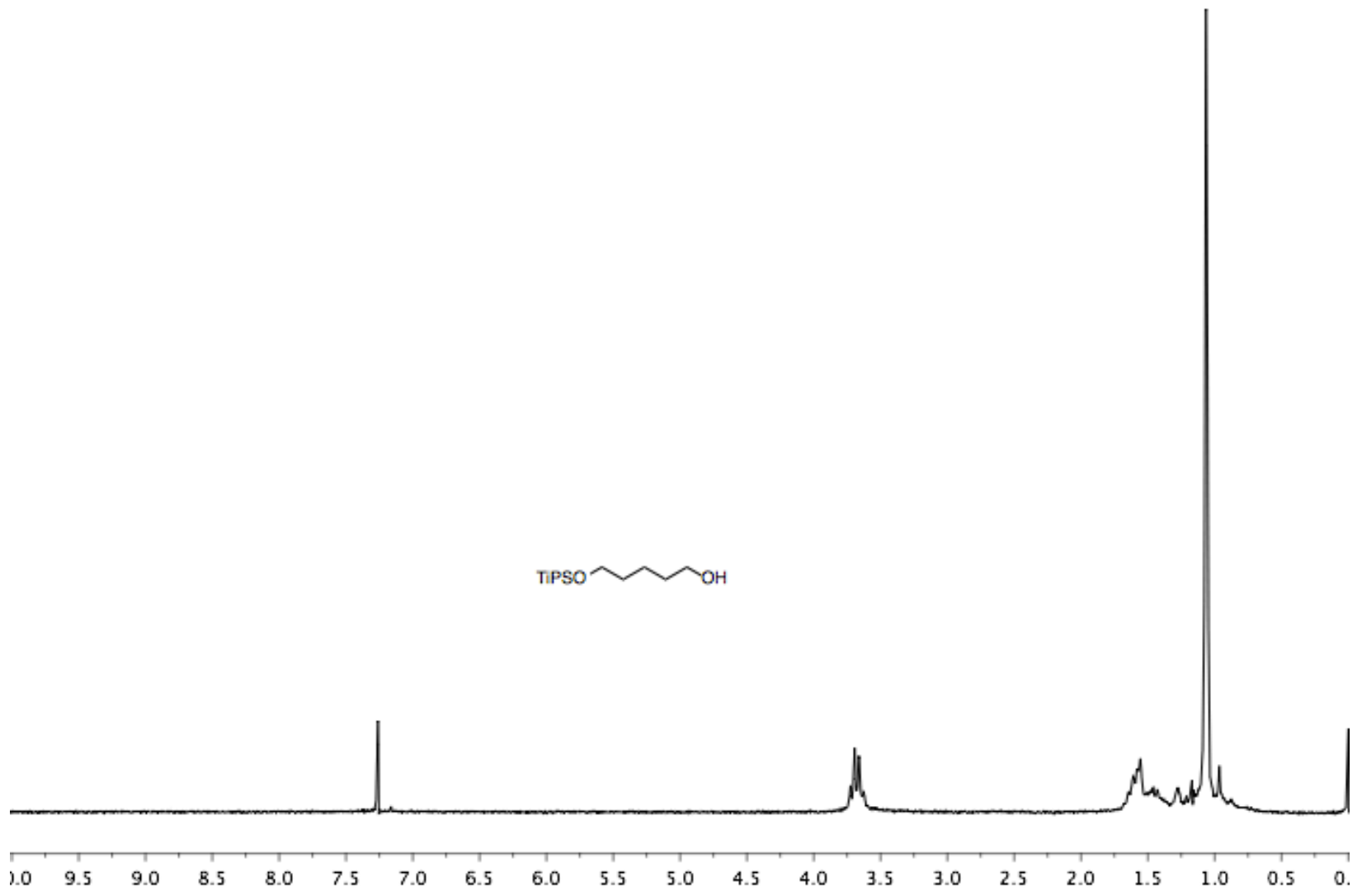




\section{${ }^{1} \mathrm{H}$ NMR Spectrum of $16 \mathrm{c}$}

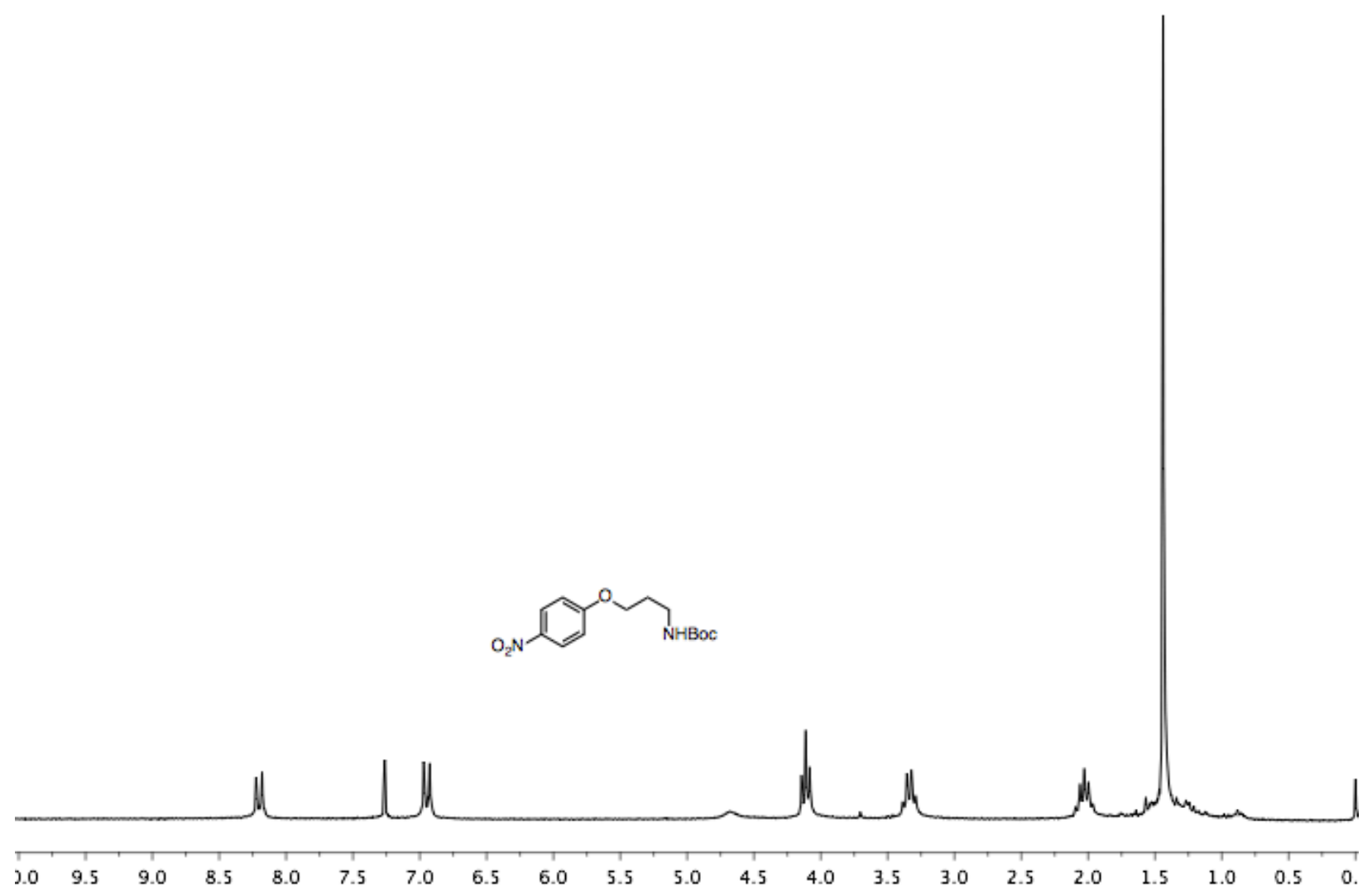

${ }^{1} \mathrm{H}$ NMR Spectrum of 16

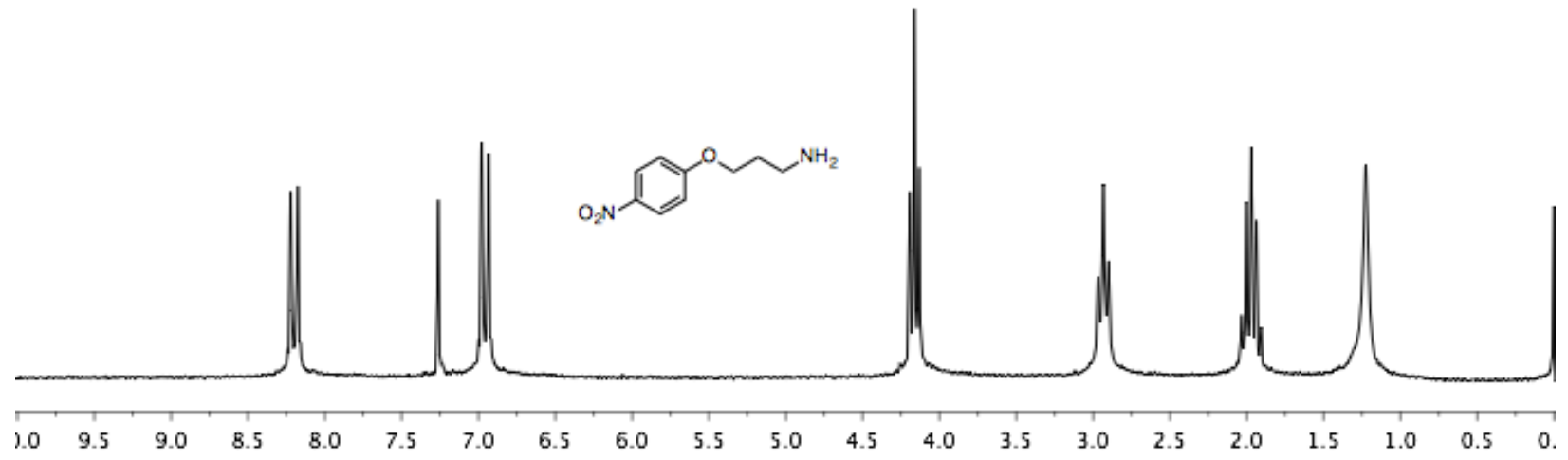


${ }^{1}$ H NMR Spectrum of 17

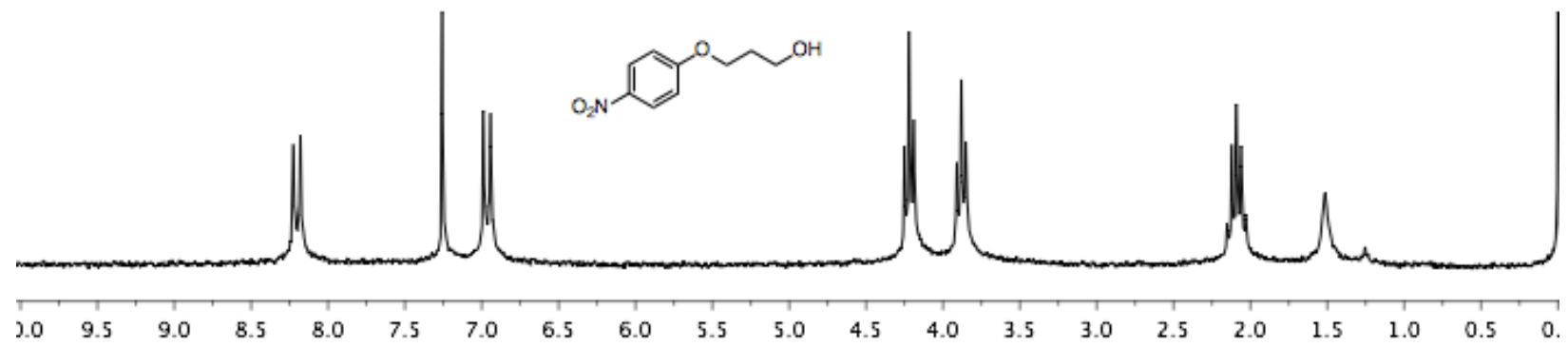

${ }^{1}$ H NMR Spectrum of 19

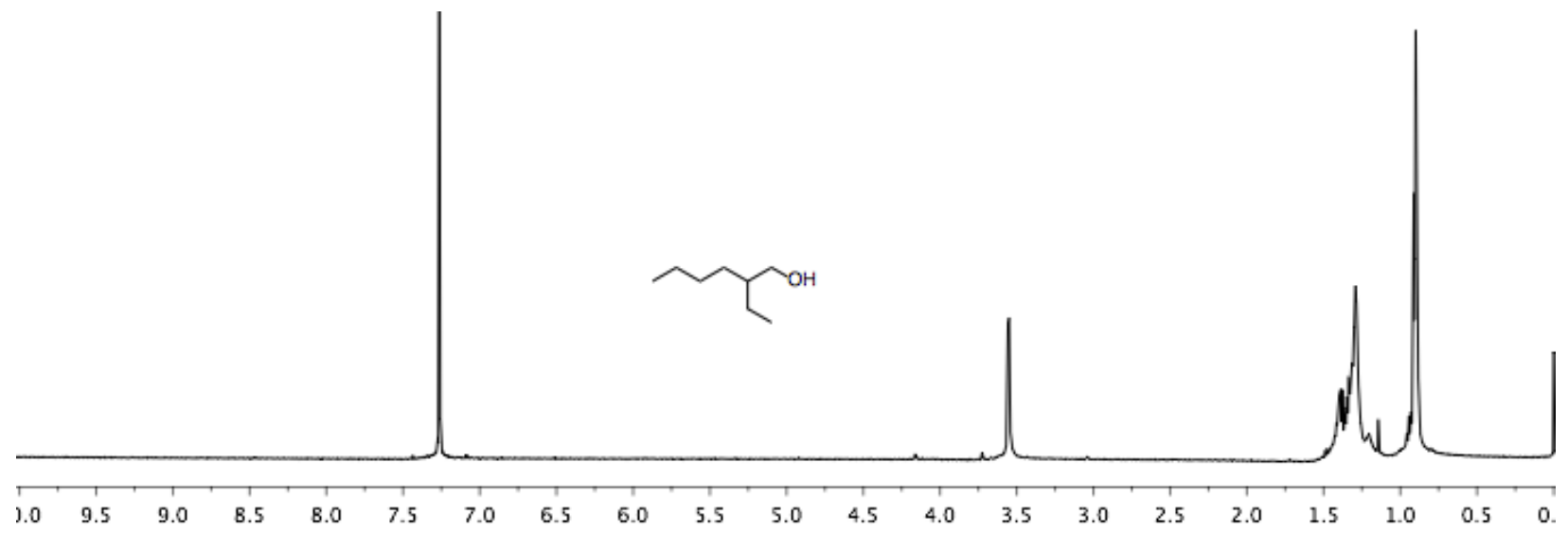

${ }^{1}$ H NMR Spectrum of 27

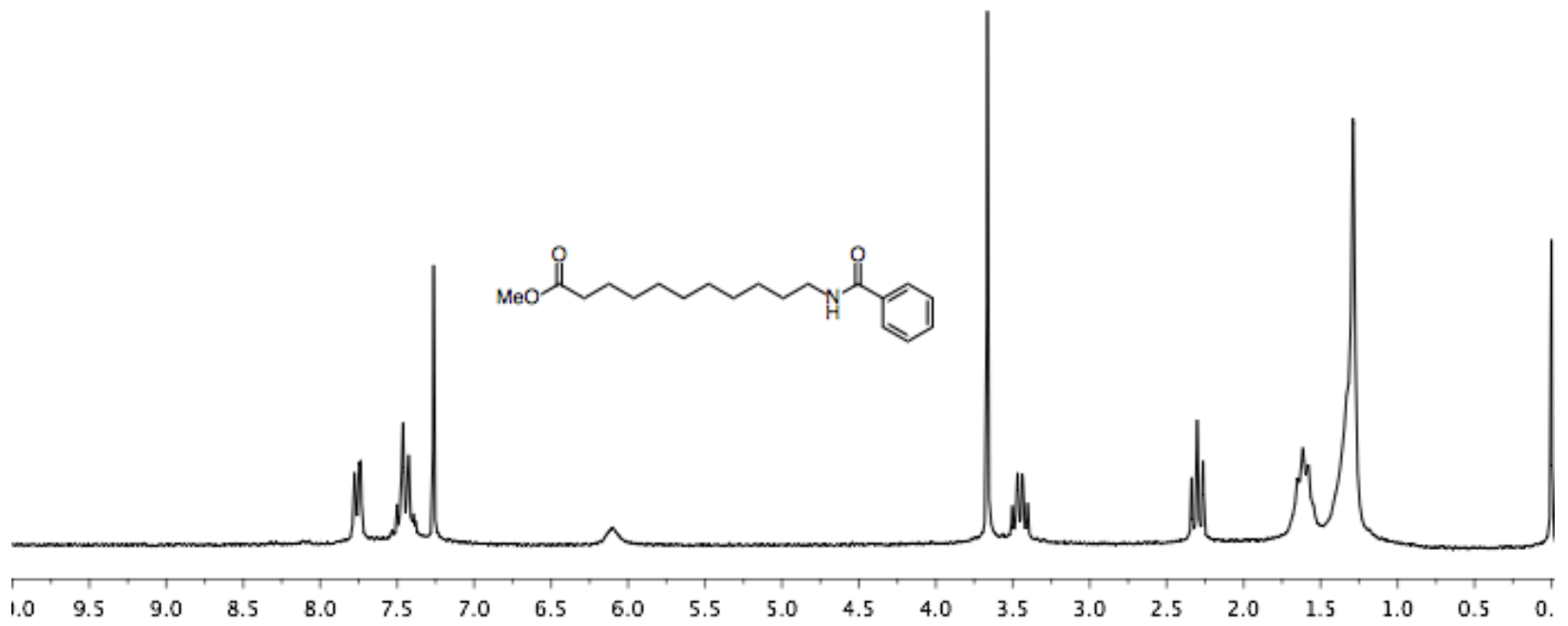


${ }^{13} \mathrm{C}$ NMR Spectrum of 27

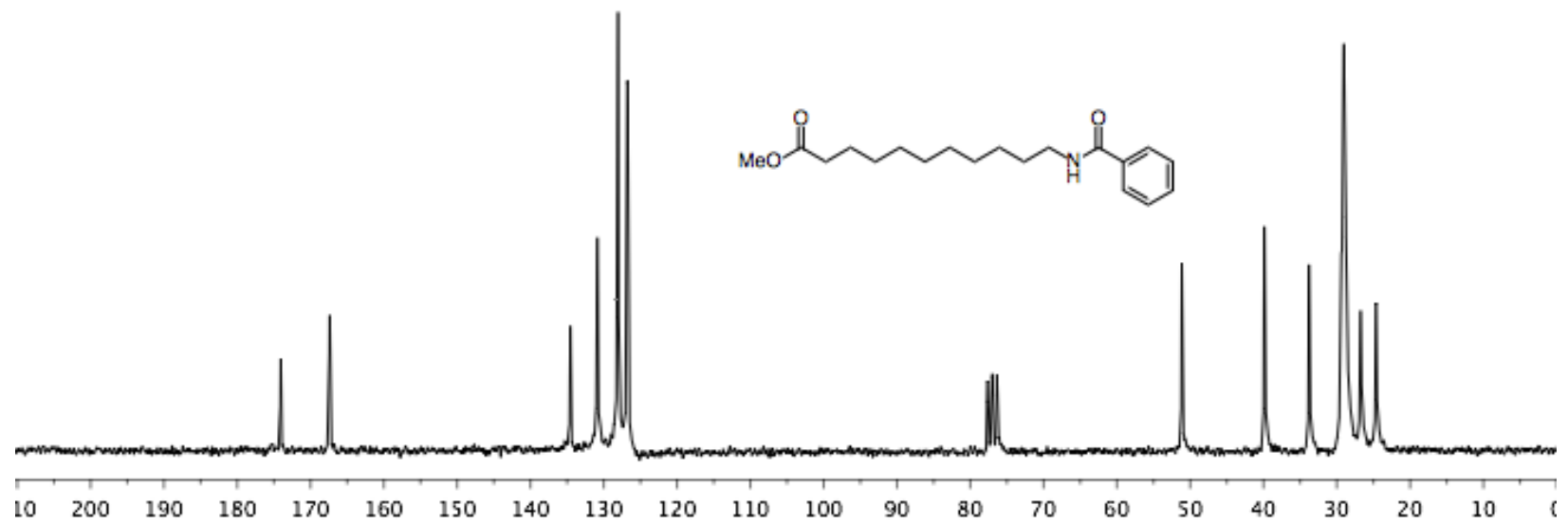

${ }^{1}$ H NMR Spectrum of 28 (crude)

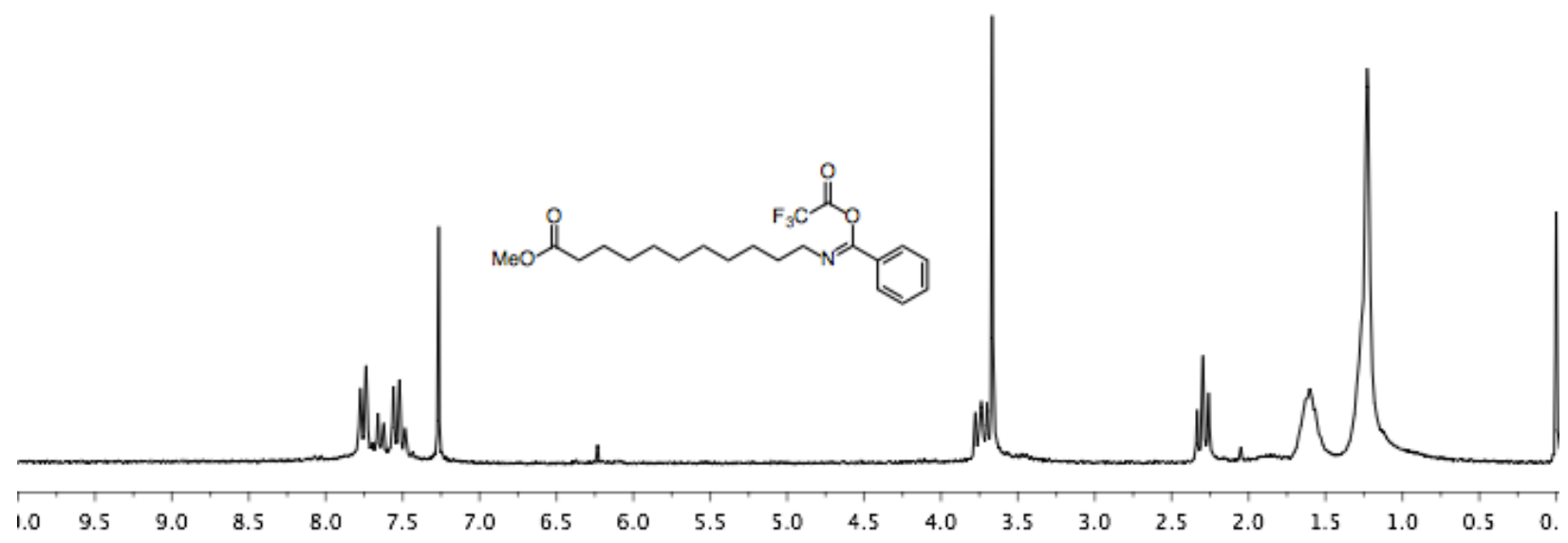

${ }^{13}$ C NMR Spectrum of 28 (crude)

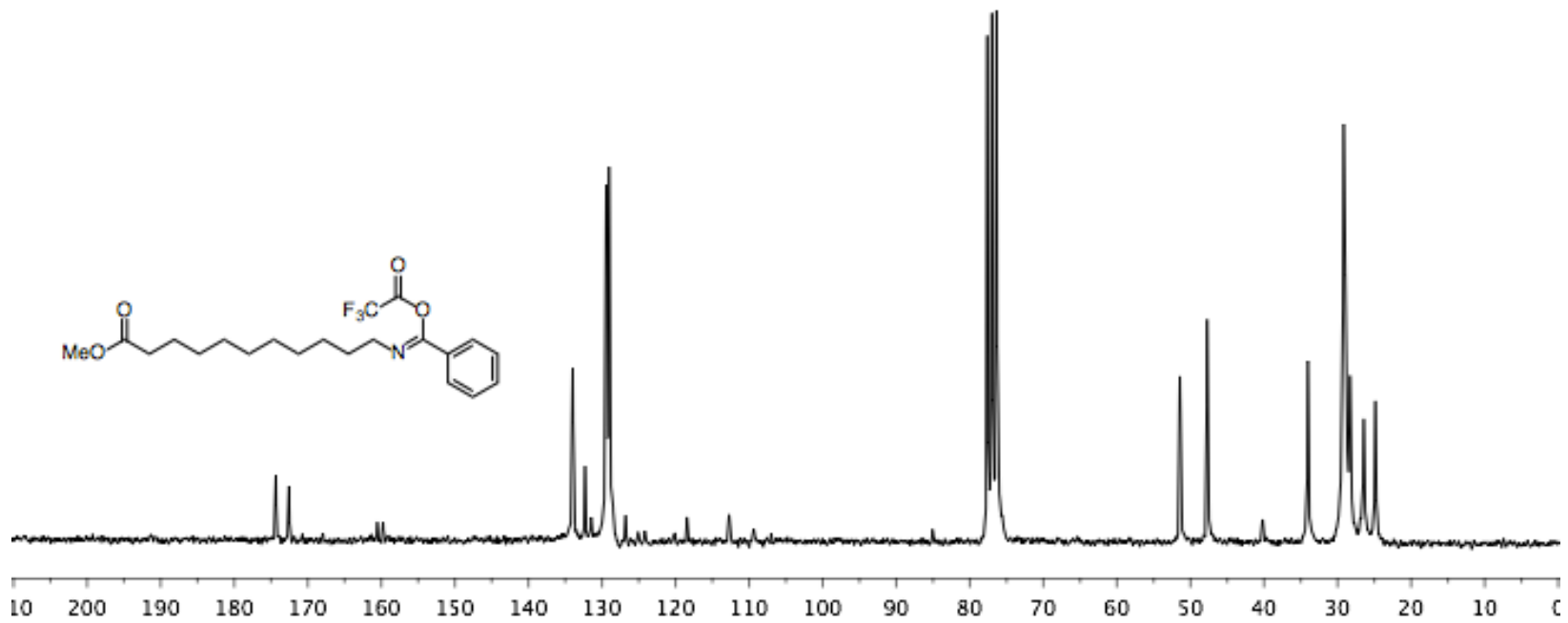


${ }^{1}$ H NMR Spectrum of S1

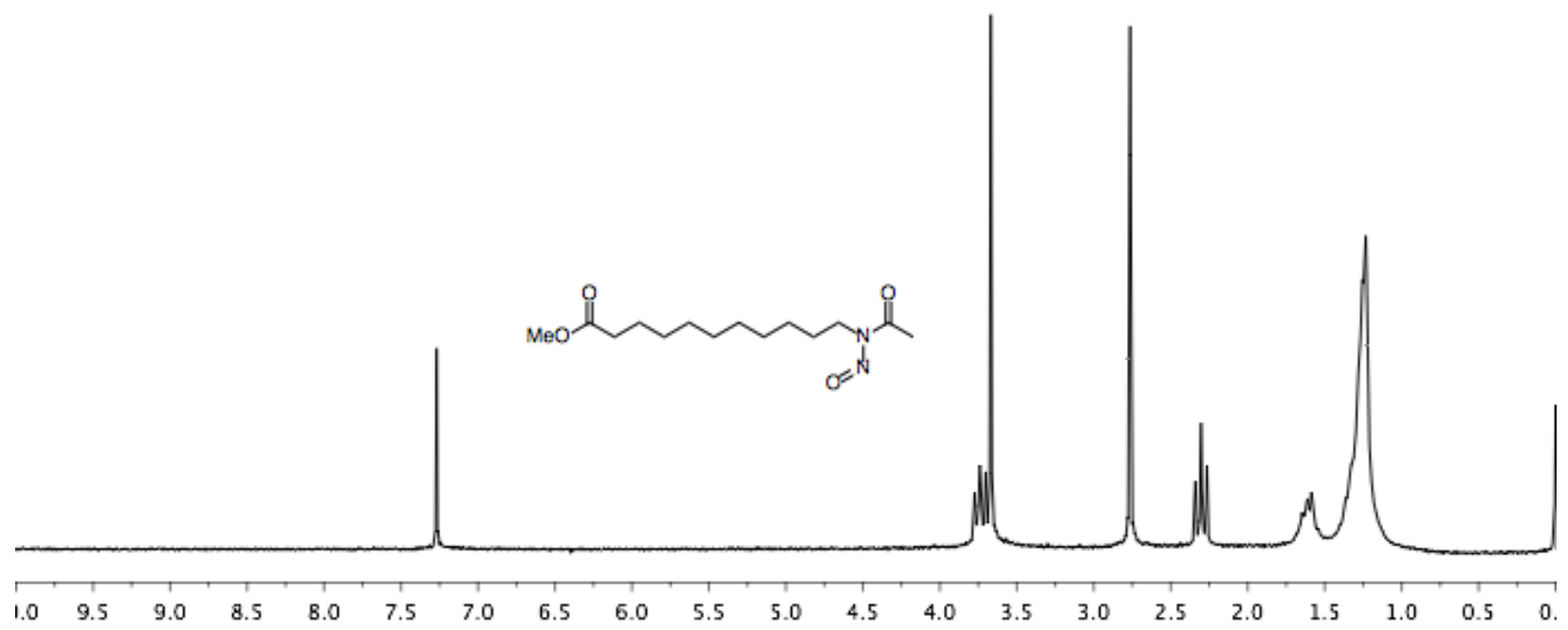

${ }^{13} \mathrm{C}$ NMR Spectrum of S1

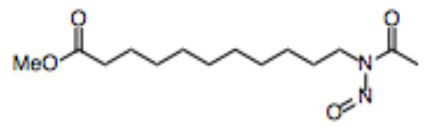

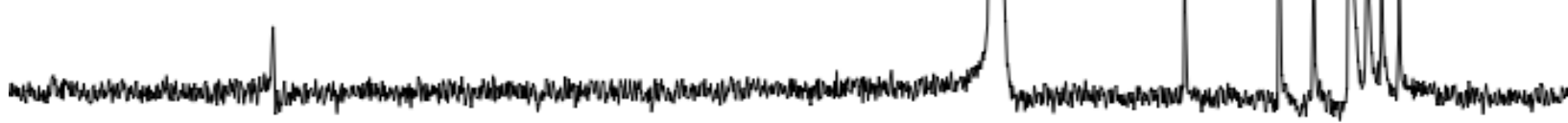

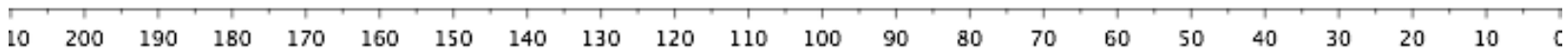

${ }^{1} \mathrm{H}$ NMR Spectrum of S1a

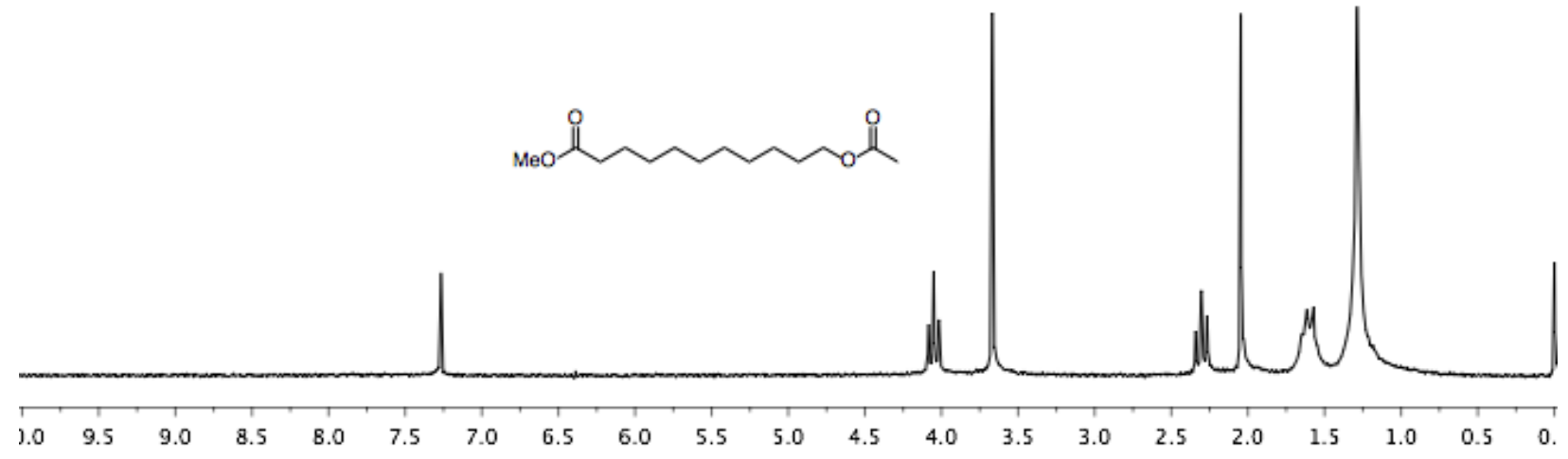


${ }^{13} \mathrm{C}$ NMR Spectrum of S1a

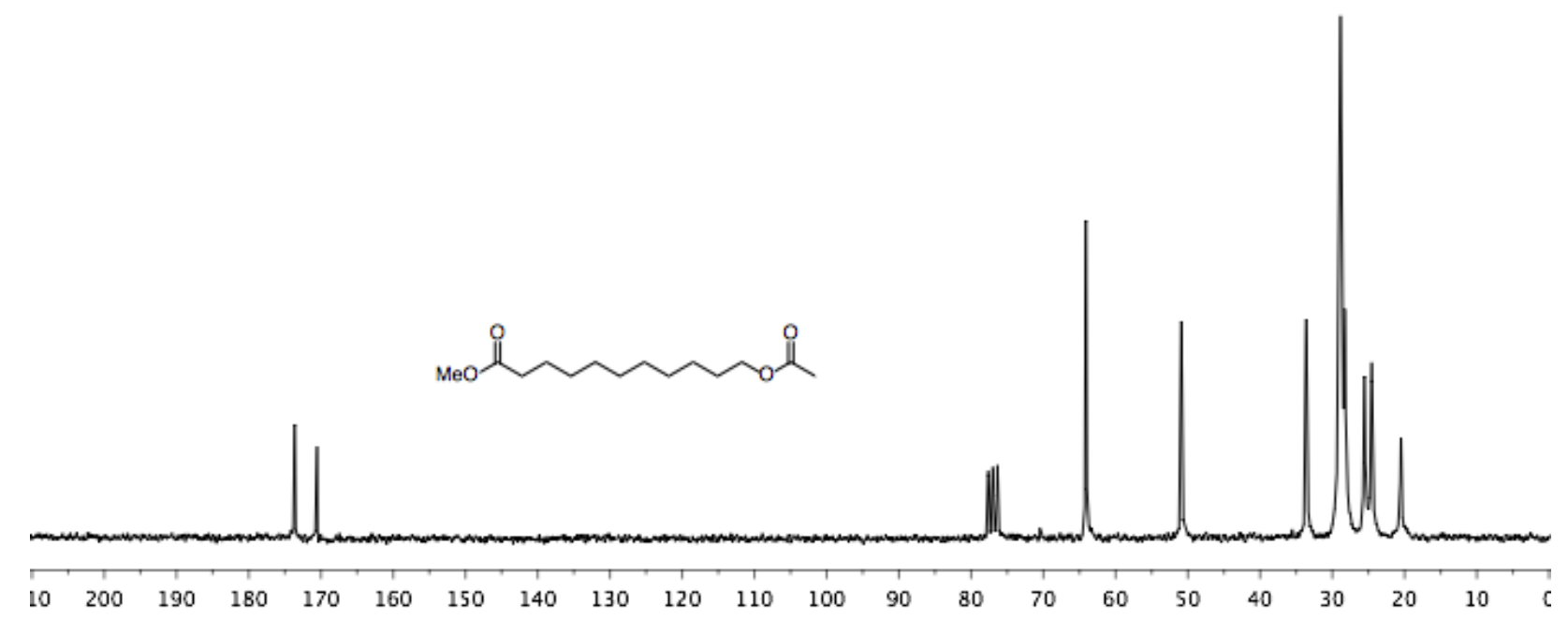

${ }^{1} \mathrm{H}$ NMR Spectrum of S2

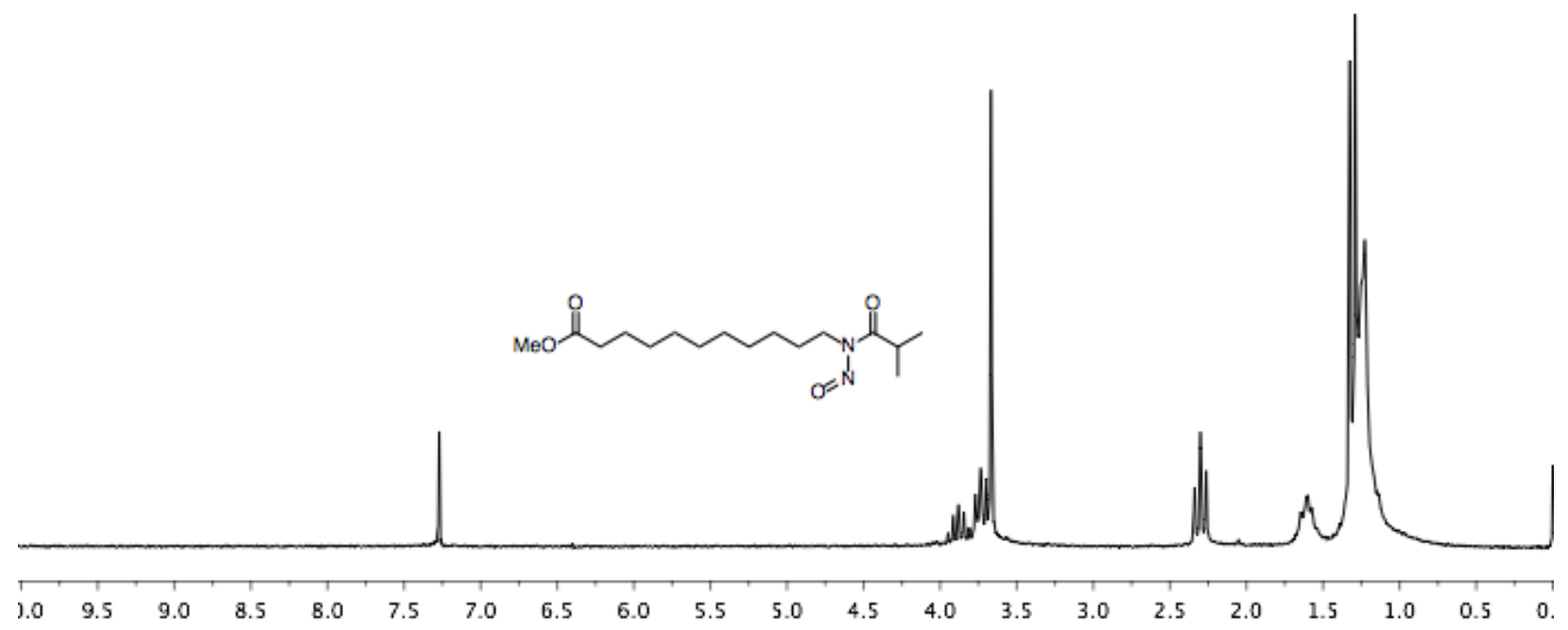


${ }^{13}$ C NMR Spectrum of S2

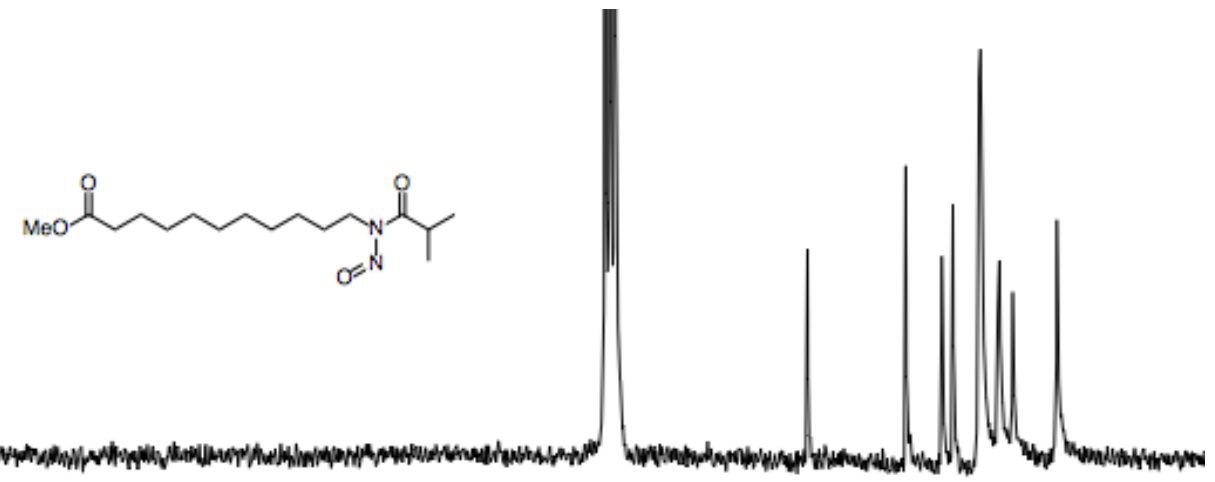

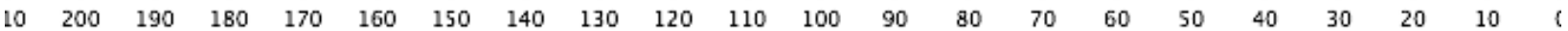

${ }^{1} \mathrm{H}$ NMR Spectrum of S2a

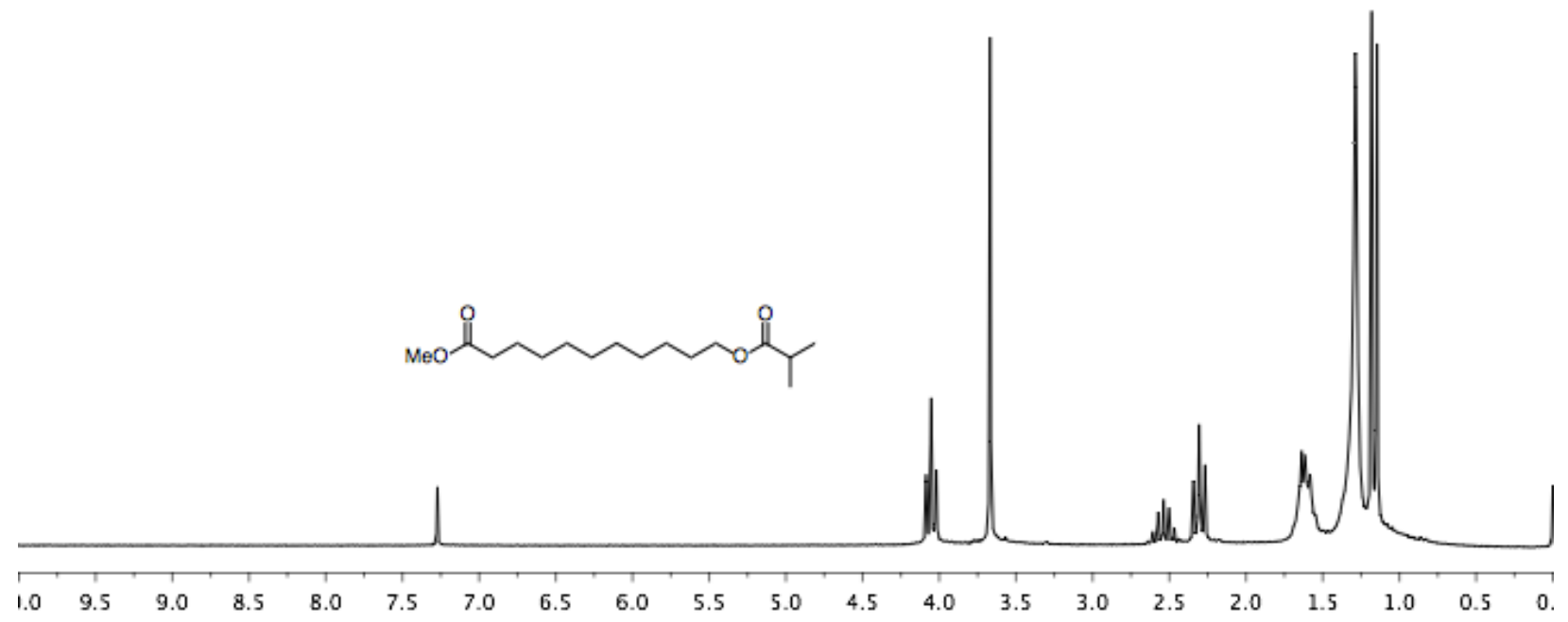


${ }^{13}$ C NMR Spectrum of S2a

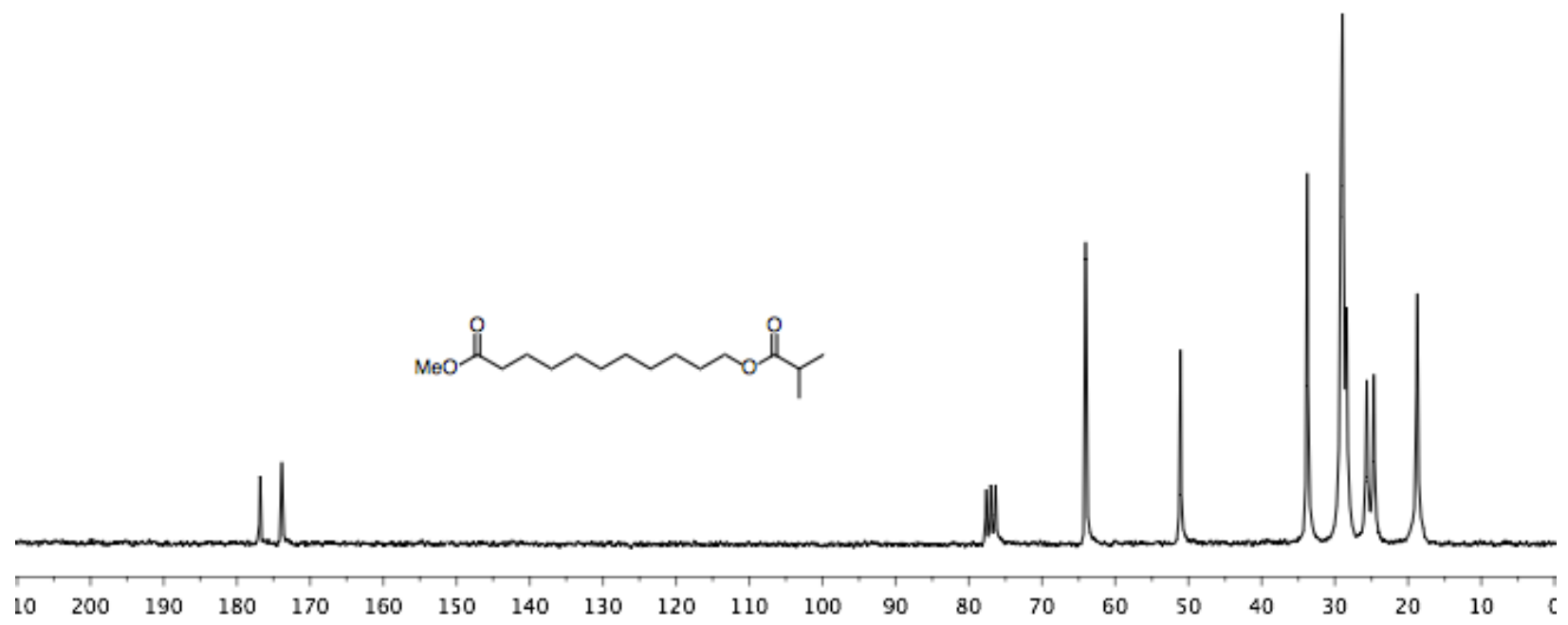

${ }^{1}$ H NMR Spectrum of S3

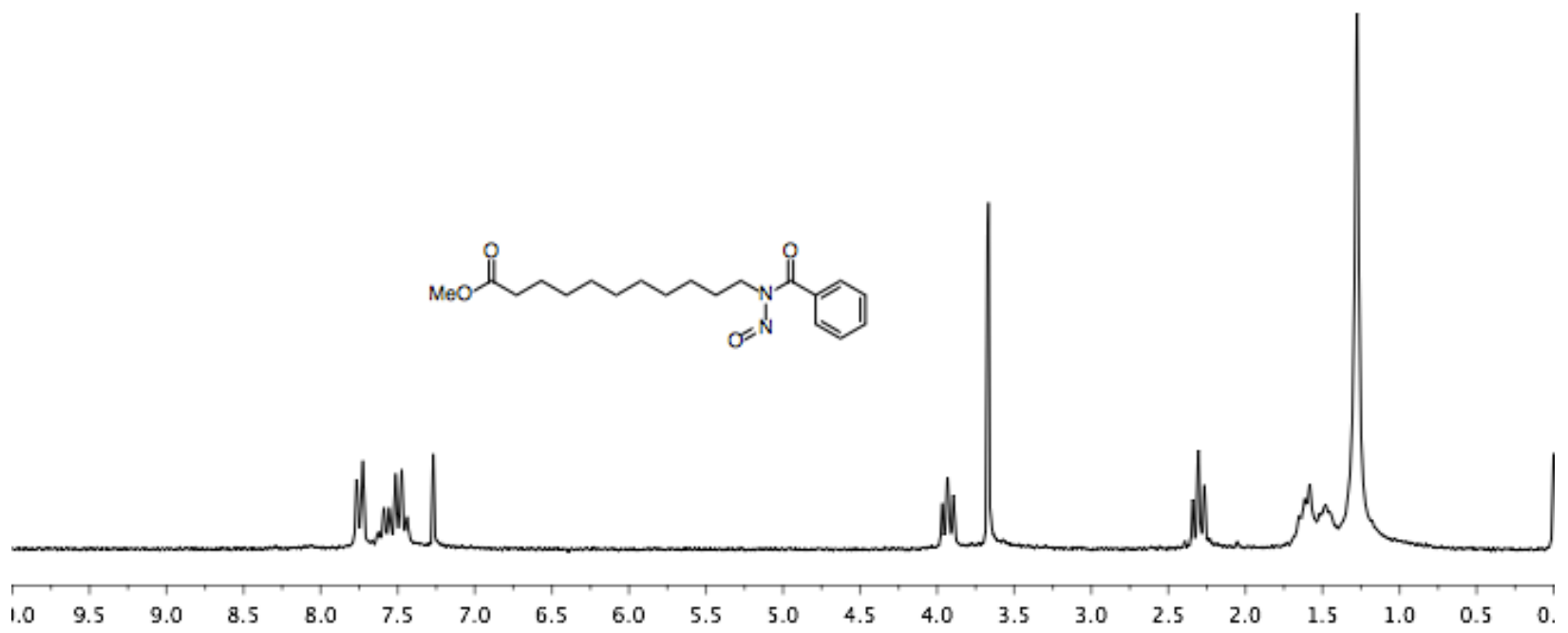

${ }^{13} \mathrm{C}$ NMR Spectrum of S3

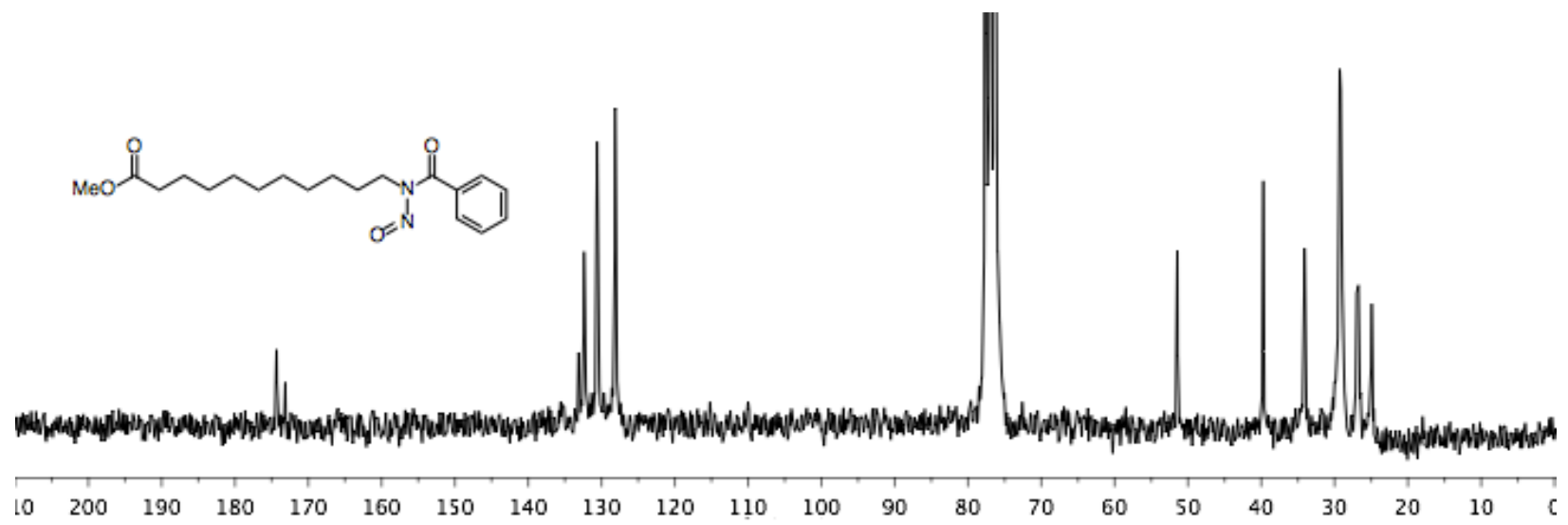




\section{${ }^{1} \mathrm{H}$ NMR Spectrum of S3a}

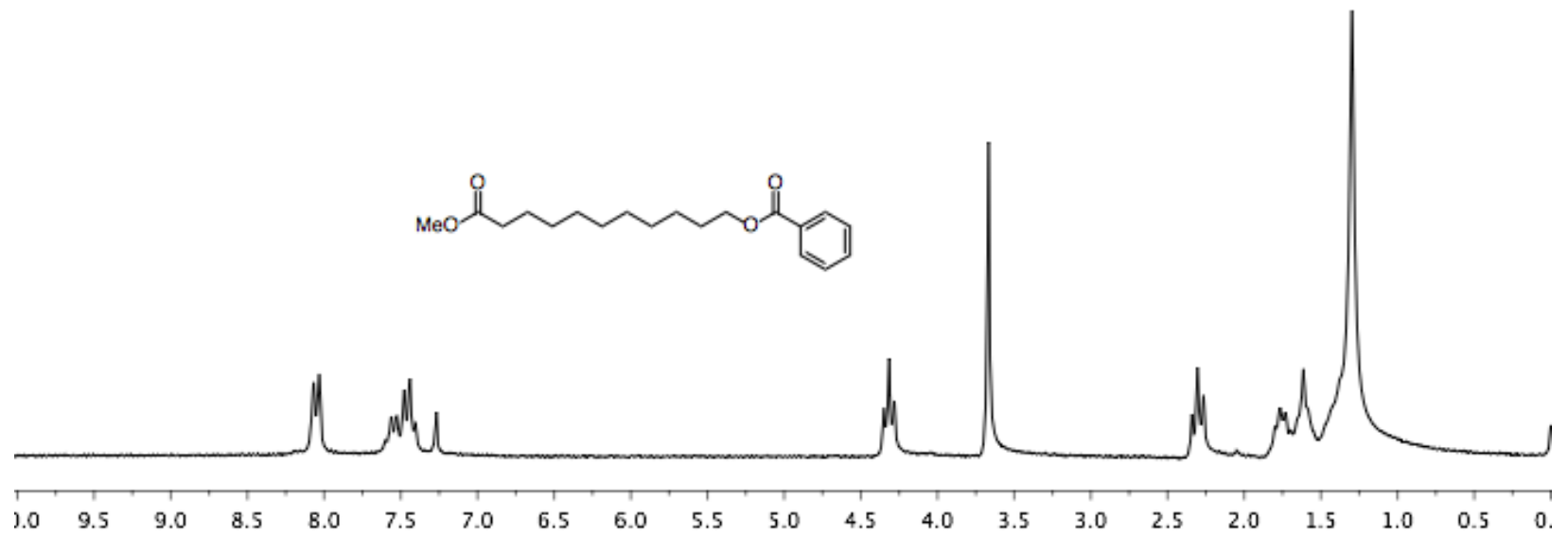

${ }^{13}$ C NMR Spectrum of S3a

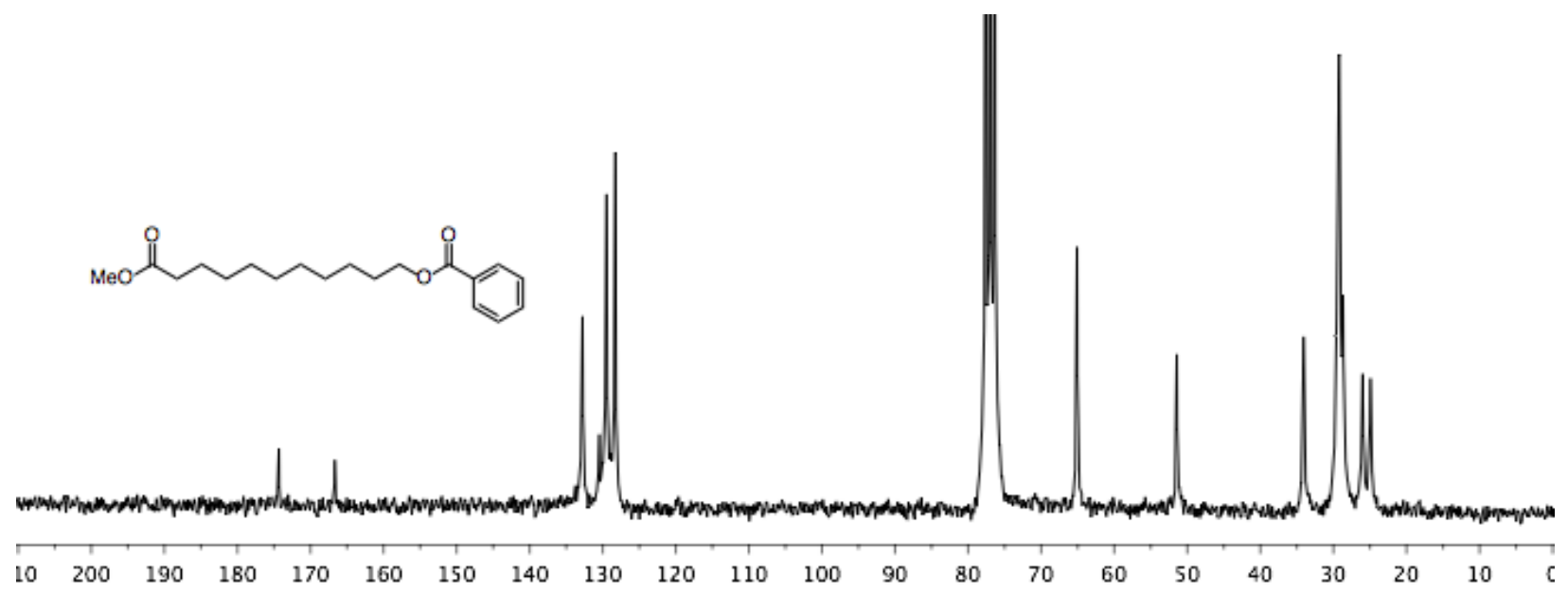

${ }^{1}$ H NMR Spectrum of S4a

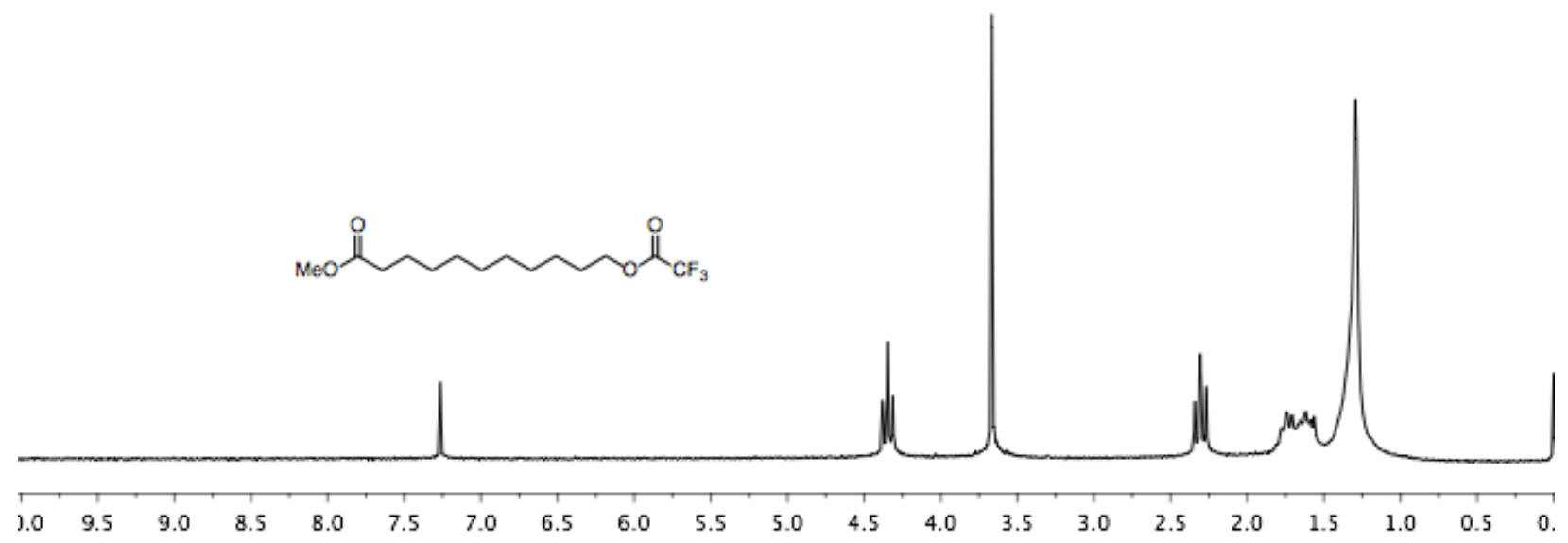


${ }^{13}$ C NMR Spectrum of S4a

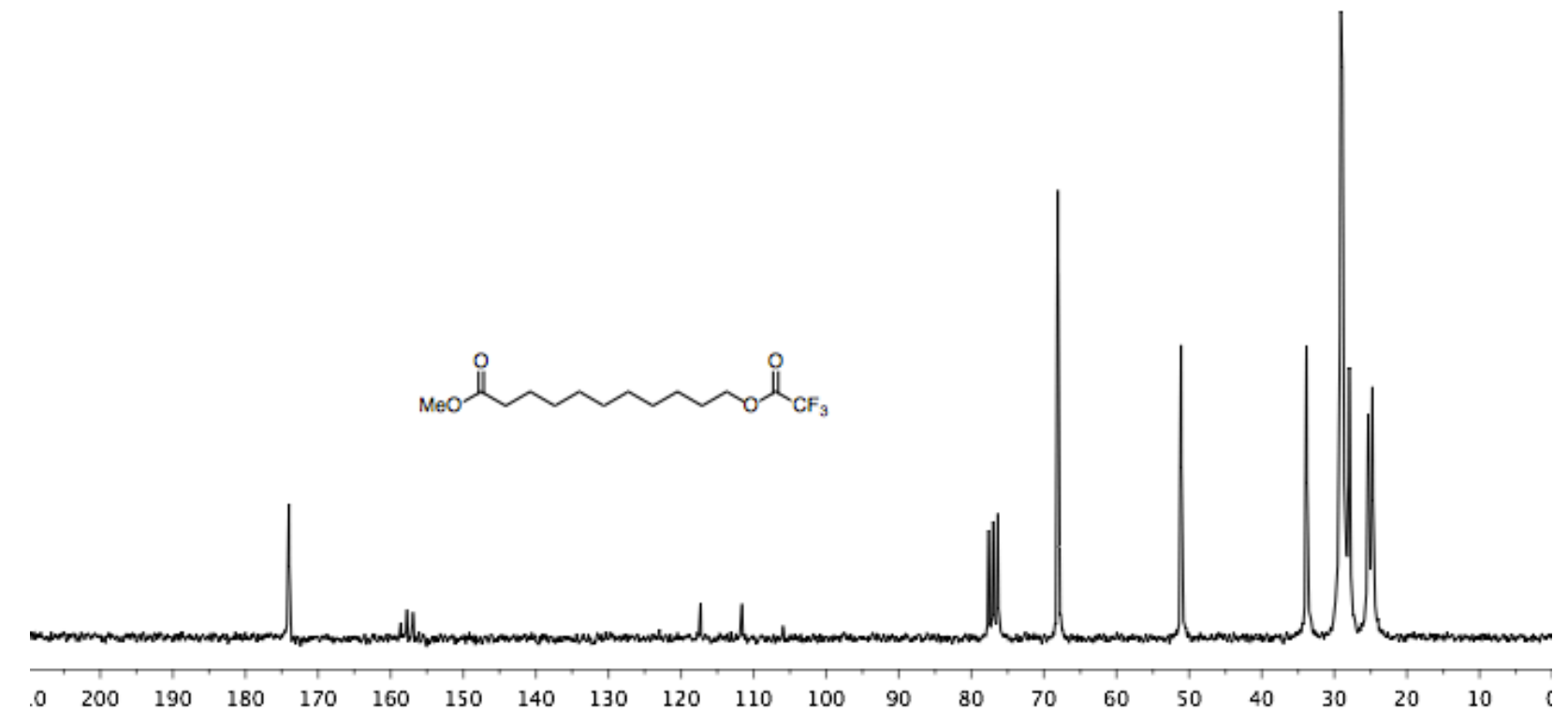

${ }^{1}$ H NMR Spectrum of S5

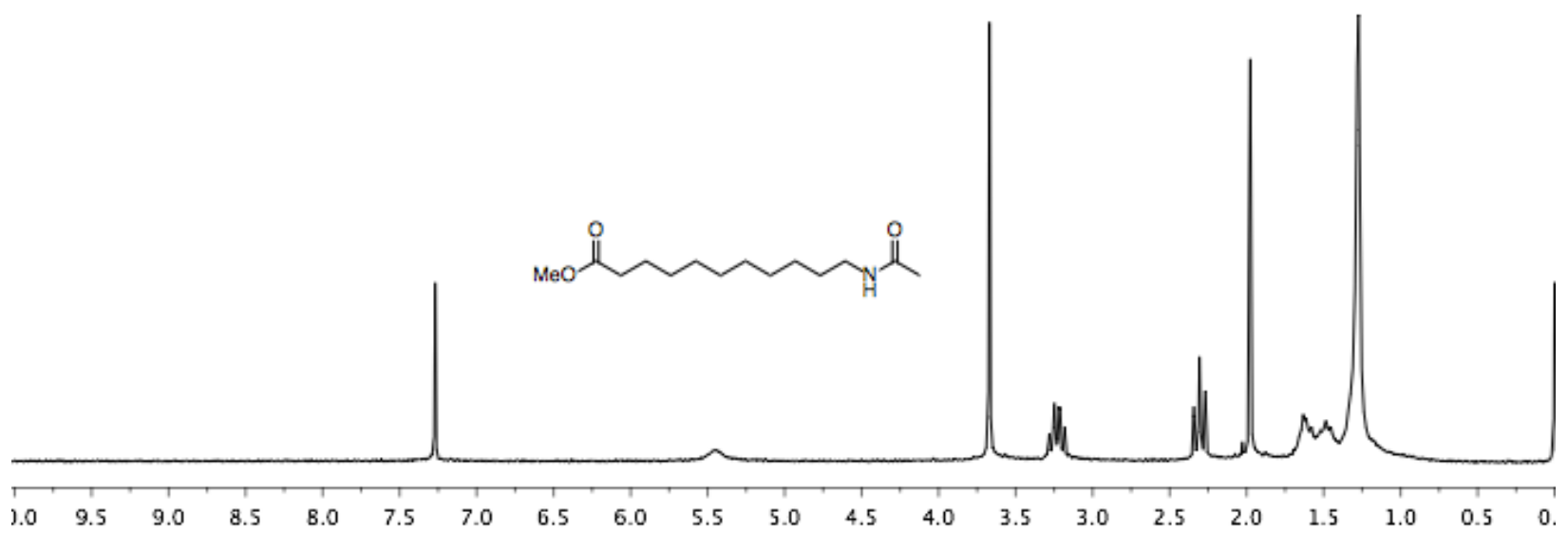


${ }^{13} \mathrm{C}$ NMR Spectrum of S5

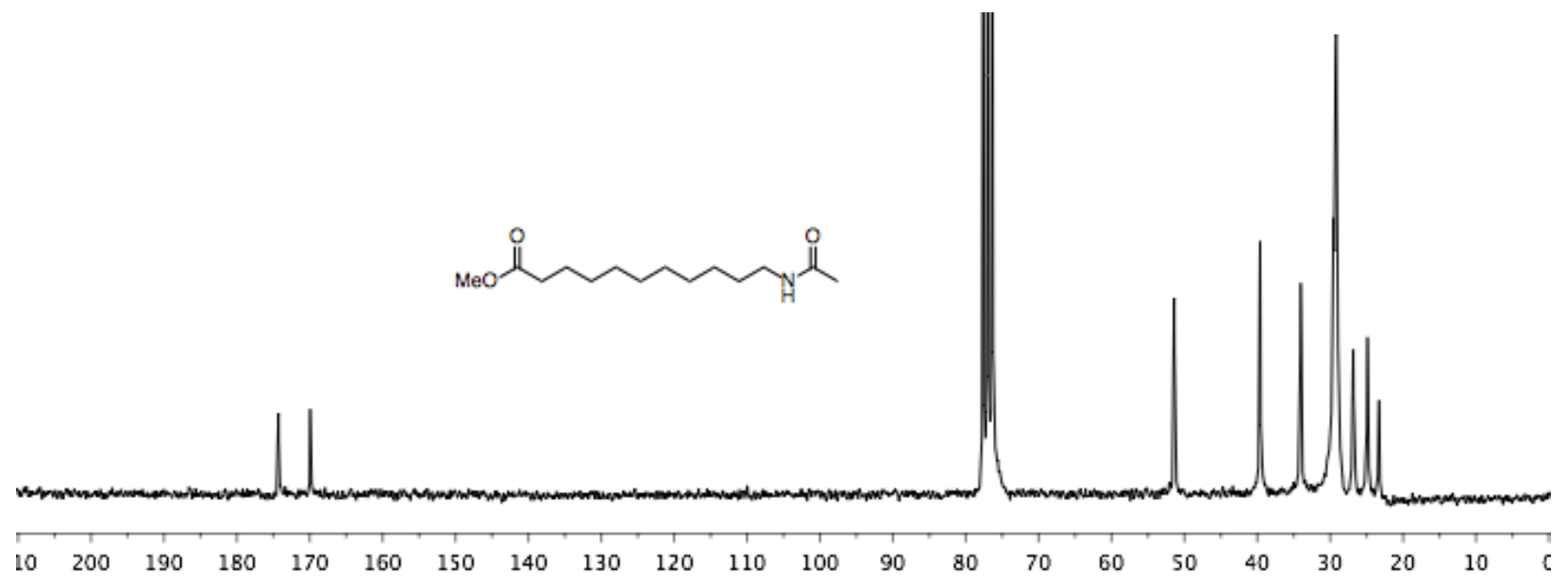

${ }^{1} \mathrm{H}$ NMR Spectrum of S6

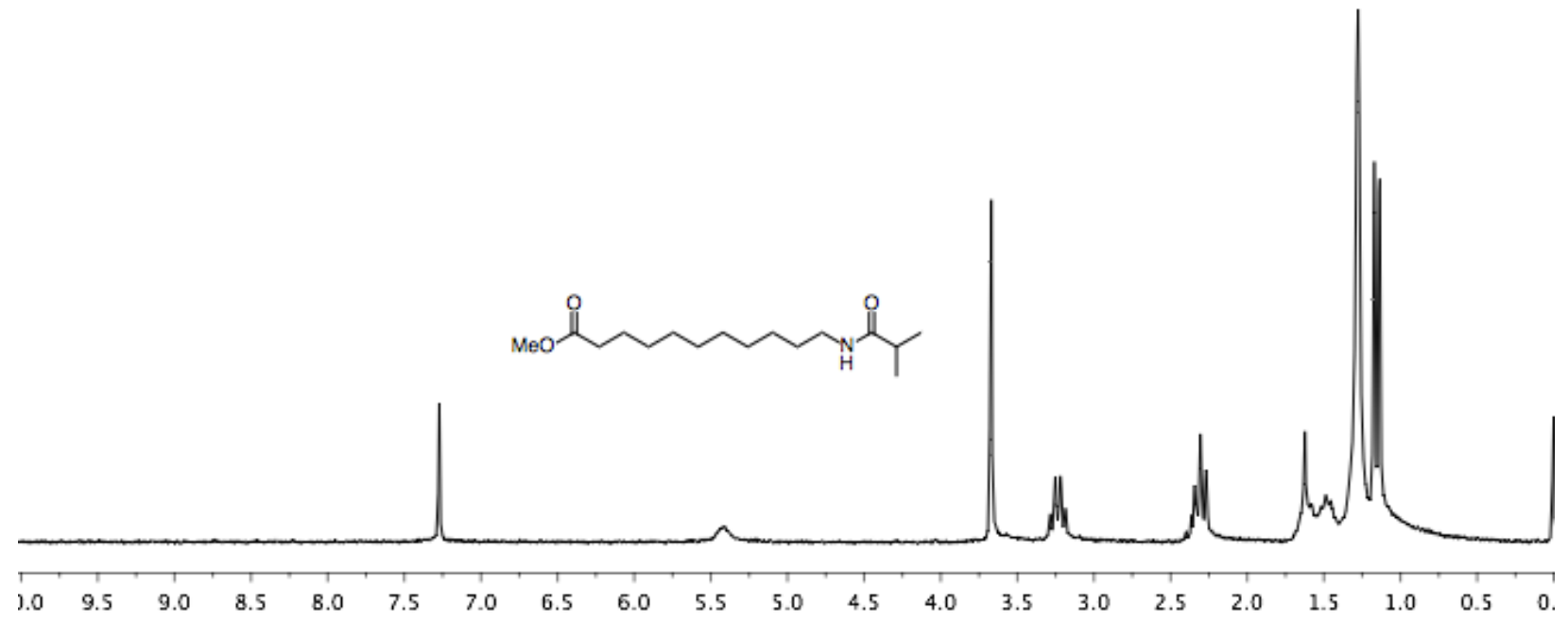


${ }^{13} \mathrm{C}$ NMR Spectrum of S6
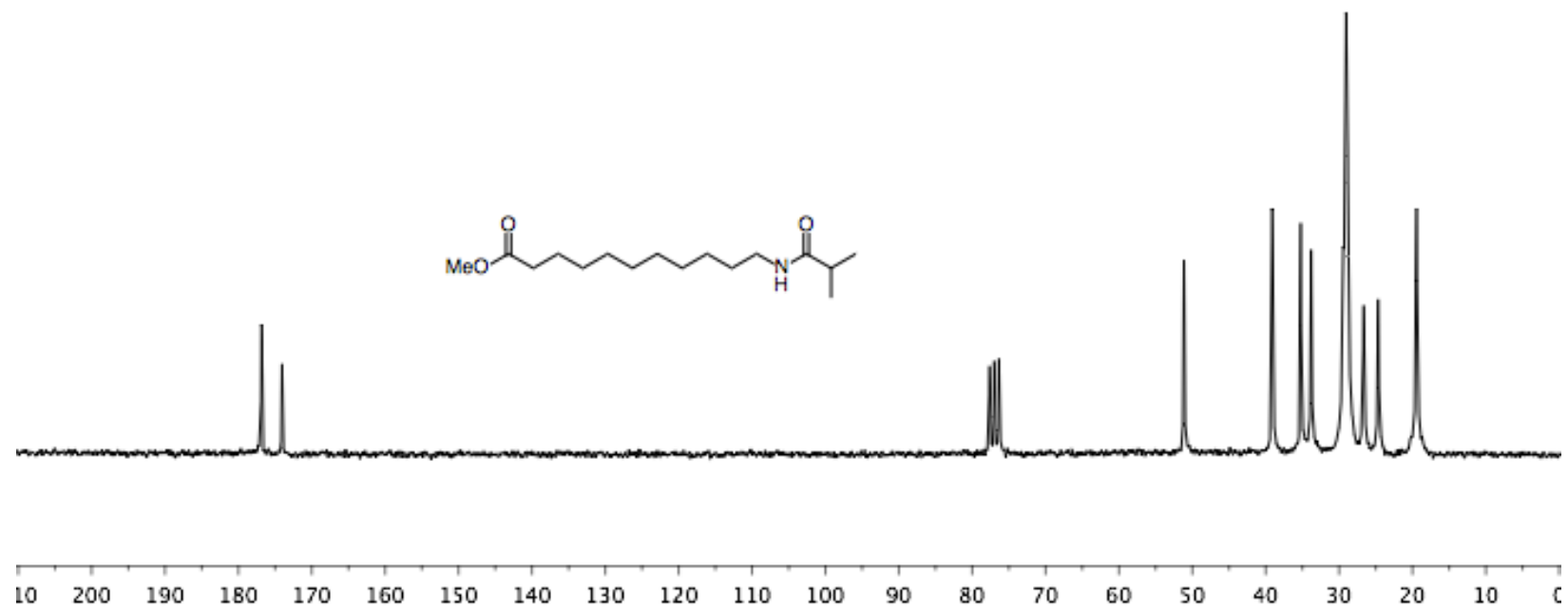

${ }^{1} \mathrm{H}$ NMR Spectrum of S7

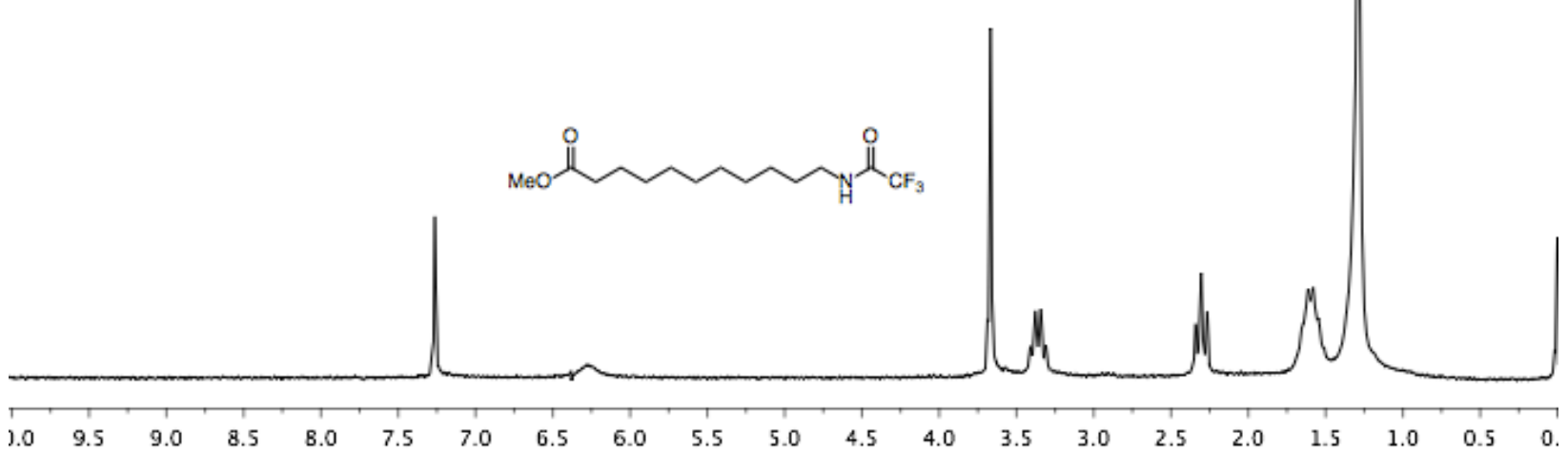

